\title{
Localization theorems in topological Hochschild homology and topological cyclic homology
}

\author{
ANDREW J BLUMBERG \\ MichaEl A MANDELL
}

\begin{abstract}
We construct localization cofibration sequences for the topological Hochschild homology $(T H H)$ and topological cyclic homology $(T C)$ of small spectral categories. Using a global construction of the $T H H$ and $T C$ of a scheme in terms of the perfect complexes in a spectrally enriched version of the category of unbounded complexes, the sequences specialize to localization cofibration sequences associated to the inclusion of an open subscheme. These are the targets of the cyclotomic trace from the localization sequence of Thomason-Trobaugh in $K$-theory. We also deduce versions of Thomason's blow-up formula and the projective bundle formula for $T H H$ and $T C$.
\end{abstract}

19D55; 14F43

\section{Introduction}

Algebraic $K$-theory provides a powerful and subtle invariant of schemes. The $K-$ theory of a scheme encodes many of its arithmetic and algebraic properties, captures information about its geometry and singularities, and is closely connected to its étale and motivic cohomology. One of the fundamental underpinnings of the subject is the localization theorem of Thomason and Trobaugh [43, 7.4], which for a quasiseparated quasicompact scheme $X$ provides a cofibration sequence of (nonconnective) $K$-theory spectra

$$
K(X \text { on }(X-U)) \longrightarrow K(X) \longrightarrow K(U) \longrightarrow \Sigma K(X \text { on }(X-U))
$$

for $U$ a quasicompact open subscheme contained in $X$. Here $K(X$ on $(X-U))$ denotes the $K$-theory of the category of perfect complexes on $X$ which are supported on the complement of $U$ in $X$. This localization sequence and the closely related Mayer-Vietoris sequence for $K$-theory allow global assembly of local information.

Keller [25] constructed the analogue of the Thomason-Trobaugh localization sequence for Hochschild homology $(H H)$ and for the variants of cyclic homology, including negative cyclic homology $\left(H C^{-}\right)$. The Dennis trace (or Chern character) connects 
the localization sequence in $K$-theory to the localization sequence in $H^{-}$. Using this, together with generalizations to blow-ups along regular sequences and Hironaka's resolution of singularities, Cortiñas, Haesemeyer, Schlichting and Weibel $[7 ; 8 ; 9]$ recently resolved Weibel's conjecture bounding below the negative $K$-groups and Vorst's conjecture that $K_{d+1}$-regularity implies regularity, for finite-type schemes of dimension $d$ over a field of characteristic zero.

The purpose of this paper is to generalize Keller's localization sequences to topological Hochschild homology (THH) and topological cyclic homology (TC). Over the course of the last two decades, $T H H$ and $T C$ have revolutionized $K$-theory computations. Roughly, topological Hochschild homology for a ring is obtained by promoting the ring to a ring spectrum and substituting the smash product of spectra for the tensor product of rings in the Hochschild complex; see Bökstedt [3]. The THH spectrum comes with a "cyclotomic" structure (which involves an $S^{1}$-action and extra structure maps), and for each prime $p$, topological cyclic homology is then defined as a certain homotopy limit over the fixed point spectra. The Dennis trace map lifts to a "cyclotomic trace" map from $K$-theory to $T C$ by work of Bökstedt, Hsiang and Madsen [4], and McCarthy [35] showed that this captures all the relative information at $p$ for surjections with nilpotent kernel, just as $\mathrm{HC}^{-}$does rationally; see Goodwillie [18]. Starting from Quillen's computation of the $K$-theory of finite fields, Hesselholt and Madsen have used $T C$ to make extensive computations in $K$-theory [19;20;21]. Moreover, because of the close relationship between $K$-theory and $T C$ (and analogy with $\mathrm{HC}^{-}$), this paper provides the key ingredients needed to generalize the work of Cortiñas, Haesemeyer, Schlichting and Weibel $[8 ; 9]$ to cases in characteristic $p$ where resolution of singularities holds. Geisser and Hesselholt have already started applying the results of this paper in this direction [17].

Between $T C$ and $T H H$ is an intermediate theory called $T R$, whose homotopy groups have the structure of a Witt complex (the structure whose universal example is the de Rham-Witt complex of Bloch, Deligne and Illusie). The Hesselholt-Madsen computations proceed by studying this structure on $T R$. Hesselholt has observed that in all known examples, the de Rham-Witt complex has the same relationship to $T R$ that Milnor $K$-theory has to algebraic $K$-theory. This led Geisser and Hesselholt to conjecture an "additive" motivic spectral sequence converging to a modified version of $T R$ with edge homomorphism the universal map from the de Rham-Witt complex. Recent work of Levine [28] axiomatizes the role of localization and Mayer-Vietoris theorems in the construction of the motivic spectral sequence (see Bloch and Lichtenbaum [1] and Friedlander and Suslin [15]), and such theorems for $T R$ should provide key input to the construction of this conjectural "additive" motivic spectral sequence. We prove the following results in this direction. 
Theorem 1.1 Let $X$ be a quasicompact and semiseparated scheme. For a quasicompact open subscheme $U$, there are homotopy cofibration sequences

$$
\begin{gathered}
T H H(X \text { on }(X-U)) \longrightarrow T H H(X) \longrightarrow T H H(U) \longrightarrow \Sigma T H H(X \text { on }(X-U)), \\
T R(X \text { on }(X-U)) \longrightarrow T R(X) \longrightarrow T R(U) \longrightarrow \Sigma T R(X \text { on }(X-U)), \\
T C(X \text { on }(X-U)) \longrightarrow T C(X) \longrightarrow T C(U) \longrightarrow \Sigma T C(X \text { on }(X-U)),
\end{gathered}
$$

where $\operatorname{THH}(X$ on $(X-U))$ denotes the THH of the spectral category of perfect complexes on $X$ which are supported on $X-U$.

For quasicompact open subschemes $U, V$ with $X=U \cup V$, the squares

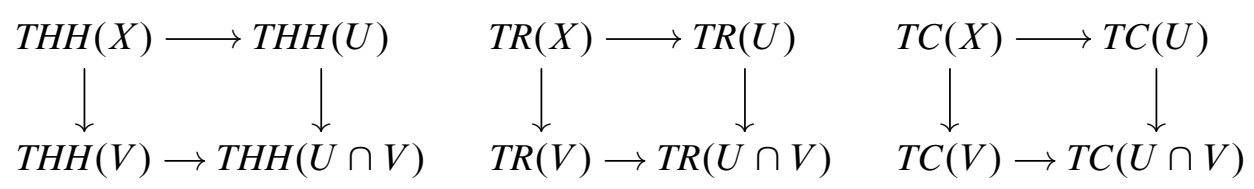

are homotopy cocartesian.

In the statement, a scheme is semiseparated [43, B.7] when it has a basis of affine open subsets whose intersections are also affine. Semiseparated is a slightly stronger condition than quasiseparated which means that it has a basis of affine open subsets whose intersection is a finite union of affine open subsets. If a scheme has an ample family of line bundles then it is semiseparated [43, B.7].

Geisser and Hesselholt [16] proved the second statement in Theorem 1.1 for $\mathrm{THH}$ of rings and used it to define THH of quasicompact quasiseparated schemes in terms of Thomason's hypercohomology construction [41, 1.33]. The relative term $T H H(X$ on $(X-U))$ does not have an intrinsic description in the context of the Geisser-Hesselholt definition of $T H H$. Here we describe it in terms of a construction of THH for spectral categories, ie, categories enriched over symmetric spectra, the stable homotopy theory refinement of DG-categories.

Dundas and McCarthy [13] generalized Bökstedt's construction of THH to small spectral categories. We build on the foundations there and study more general invariance properties; see in particular Theorems 5.9 and 5.12 below. We use these invariance properties to generalize the localization theorem of Keller to the setting of spectral categories. Roughly, we show that the $T H H$ of a triangulated quotient is the cofiber on $\mathrm{THH}$; Theorems 7.1 and 7.2 provide precise statements. Although we work in the context of spectral categories, our localization theorem specializes to the setting of DG-categories, as small DG-categories may be functorially converted to small spectral categories with the same objects and spectral refinements of the Hom complexes; see for example Schwede and Shipley [37, Section 6] or Dugger and Shipley [12, Appendix A], 
among others. Just as $T H H$ of a ring captures much more torsion information than $H H$ of the ring, $T H H$ provides a richer invariant of a DG-category than $H H$. Using an appropriate functor from small DG-categories to small spectral categories and DG-categories, we make the following observation at the end of Section 3.

Theorem 1.2 The constructions of THH, TR and TC as defined in Section 3 are functors from the category of small $D G$-categories and $D G$-functors to the stable category.

We define $T H H$ of a scheme in terms of a spectral category refinement $\mathcal{D}_{\text {parf }}^{S}(X)$ of the DG-category quotient $\mathcal{D}_{\text {parf }}^{\mathrm{DG}}(X)$ modeling the derived category of perfect complexes. In Section 8, we prove the following consistency theorem that compares this definition to the definition of Geisser and Hesselholt.

Theorem 1.3 Let $X$ be a quasicompact and semiseparated scheme, and $\mathcal{D}_{\text {parf }}^{S}(X)$ a spectral category refinement of $\mathcal{D}_{\text {parf }}^{\mathrm{DG}}(X)$. Then $\operatorname{THH}\left(\mathcal{D}_{\text {parf }}^{S}(X)\right)$ is equivalent to the Thomason hypercohomology of the presheaf of symmetric spectra $U \mapsto T H H\left(\mathcal{O}_{U}\right)$ on the small Zariski site of $X$.

This theorem in particular constructs a trace map from the $K$-theory of the scheme to $\operatorname{THH}\left(\mathcal{D}_{\text {parf }}^{S}(X)\right)$ and $T C\left(\mathcal{D}_{\text {parf }}^{S}(X)\right)$. In Section 9, we show that the trace map factors through Thomason and Trobaugh's Bass' nonconnective $K$-theory spectrum using their spectral version of Bass' fundamental theorem. In Section 10, we give a direct construction of the trace for $\mathcal{D}_{\text {parf }}^{S}(X)$ that does not use the hypercohomology construction.

In addition to Theorem 1.1, we also establish $T H H$ and $T C$ versions of two classical geometric calculations in algebraic $K$-theory using our general localization machinery. First, we prove the following formula for blow-ups along regular sequences, which already has been applied by Geisser and Hesselholt [17] to prove the characteristic $p$ analogue of Weibel's conjecture. We state the theorem using the notation of $[8$, Section 1], and prove it in Section 8.

Theorem 1.4 Let $X$ be a quasicompact and semiseparated scheme. Let $i: Y \subset X$ be a regular embedding of a closed subscheme, $p: X \rightarrow X^{\prime}$ the blowup along $Y$, $j: Y^{\prime} \subset X^{\prime}$ the exceptional divisor, and write $q$ for the map $Y^{\prime} \rightarrow Y$. Then the squares
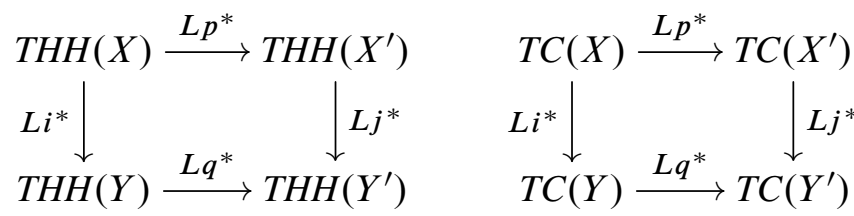

are homotopy cocartesian. 
We also prove a projective bundle theorem $[43,4.1,7.3]$ in Section 8.

Theorem 1.5 Let $X$ be a quasicompact and semiseparated scheme. Let $\mathcal{E}$ be an algebraic vector bundle of rank $r$ over $X$, and let $\pi: \mathbb{P} \mathcal{E}_{X} \rightarrow X$ be the associated projective bundle. Then a spectral lift of the derived functor

$$
\bigoplus_{i=0}^{r-1} \mathcal{O}_{\mathbb{P} \mathcal{E}_{X}}(-i) \otimes L \pi^{*}(-)
$$

induces weak equivalences

$$
\prod_{i=0}^{r-1} \operatorname{THH}(X) \longrightarrow \operatorname{THH}\left(\mathbb{P} \mathcal{E}_{X}\right) \text { and } \prod_{i=0}^{r-1} T C(X) \longrightarrow T C\left(\mathbb{P} \mathcal{E}_{X}\right) \text {. }
$$

The proof of the previous two theorems require the machinery of the construction of THH of small spectral categories that we develop in this paper; it is not known how to prove them using just a hypercohomology construction like that of Geisser and Hesselholt [16].

Organization of the paper In Section 2, we review the basic definitions for spectral categories (categories enriched in symmetric spectra). As indicated above, this is the appropriate setting for studying $T H H, T R$ and $T C$, and is a stable homotopy theory generalization of the setting of DG-categories. In Section 3, we review the definition of THH of small spectral categories due to Bökstedt [3] and Dundas and McCarthy [13]. Because of the work of Shipley [39], the technical hypotheses of connectivity and convergence on the input symmetric spectra that seemed necessary for the last 20 years may now be omitted. We take the viewpoint, first articulated by Dwyer and Kan, that enriched mapping spaces (or spectra) encode the "higher homotopy theory" of a category, and we view $T H H, T R$ and $T C$ as invariants of the higher homotopy theory of the category, as is $K$-theory (see Toën and Vezzosi [44] and our paper [2]). Section 4 spells out in detail the definition of the point-set category of cyclotomic spectra of orthogonal spectra. In Section 5, we list several invariance theorems for $\mathrm{THH}$ in this context. Section 6 reviews an elementary tilting argument for $T H H$, Proposition 6.2, originally due to Dennis and Waldhausen [45, page 391]. We demonstrate how to apply the tilting argument to prove powerful comparison theorems. Using these techniques, in Section 7 we prove the general localization theorems, Theorems 7.1 and 7.2, which we apply in Section 8 to prove Theorems 1.1, 1.3, 1.4 and 1.5 above. In Section 9, we extend the cyclotomic trace over Bass' nonconnective $K$-theory, using Thomason and Trobaugh's spectral version of Bass' fundamental theorem. To simplify the discussion, we use the ad hoc version of the cyclotomic trace for schemes in [16] induced by the cyclotomic 
trace for rings; Section 10 constructs a canonical version of the cyclotomic trace map for arbitrary DG-Waldhausen categories. This requires a review of Waldhausen's $S$. construction and the construction of algebraic $K$-theory. The last section gives a version of Theorem 7.1 that is more useful in the context of spectral model categories.

Acknowledgements The authors would like to thank the Department of Mathematics and the Mathematics Research Center at Stanford University and the Institute for Advanced Study for their hospitality and support while some of this work was being done. The authors would like to thank Lars Hesselholt for asking motivating questions and for sharing his ideas in this direction, as well as Christian Haesemeyer, John Rognes, Marco Schlichting, Brooke Shipley and Charles Weibel for interesting and useful conversations.

Blumberg was supported in part by an NSF postdoctoral fellowship, NSF grant DMS0111298 and a Clay Liftoff Fellowship. Mandell was supported in part by NSF grants DMS-0504069 and DMS-0804272.

\section{Review of spectral categories}

Modern constructions of the stable category with point-set level smash products allow easy generalization of the concepts of simplicial category or DG-category to the context of spectra. Symmetric spectra in particular often arise naturally as the refinement of mapping sets. In fact, symmetric ring spectra (the analogue of DG-rings) and categories enriched in symmetric spectra (the analogue of DG-categories) predated Smith's insight that the homotopy theory of symmetric spectra models the stable category. In older $K$-theory literature, they were called FSPs (or FSPs defined on spheres) and FSPs with many objects, respectively, and treatments generally included hypotheses on connectivity or convergence. A modern approach to $T H H$ and $T C$, taking advantage of Hovey, Shipley and Smith [24] and especially Shipley [39] obviates the need for any such connectivity or convergence hypotheses. In this section, we review the definition of spectral categories, and modules and bimodules over spectral categories in terms of enriched category theory.

Definition 2.1 A spectral category is a category enriched over symmetric spectra in simplicial sets. Specifically, a spectral category $\mathcal{C}$ consists of

(i) a collection of objects ob $\mathcal{C}$ (which need not be a small set),

(ii) a symmetric spectrum $\mathcal{C}(a, b)$ for each pair of objects $a, b \in \mathrm{ob} \mathcal{C}$,

(iii) a unit map $S \rightarrow \mathcal{C}(a, a)$ for each object $a \in \operatorname{ob} \mathcal{C}$, and

(iv) a composition map $\mathcal{C}(b, c) \wedge \mathcal{C}(a, b) \rightarrow \mathcal{C}(a, c)$ for each triple of objects $a, b, c \in$ $\mathrm{ob} \mathcal{C}$, 
satisfying the usual associativity and unit properties. We say that a spectral category is small when the objects ob $\mathcal{C}$ form a small set.

We emphasize that the data in (iii) and (iv) consist of point-set maps (rather than maps in the stable category) and that " $\wedge$ " denotes the point-set smash product of symmetric spectra. The definition of spectral functor between spectral categories is the usual definition of an enriched functor:

Definition 2.2 Let $\mathcal{C}$ and $\mathcal{D}$ be spectral categories. A spectral functor $F: \mathcal{C} \rightarrow \mathcal{D}$ is an enriched functor. Specifically, a spectral functor consists of

(i) a function on objects $F:$ ob $\mathcal{C} \rightarrow$ ob $\mathcal{D}$, and

(ii) a map of symmetric spectra $F_{a, b}: \mathcal{C}(a, b) \rightarrow \mathcal{D}(F a, F b)$ for each pair of objects $a, b \in \mathrm{ob} \mathcal{C}$,

which is compatible with the units and the compositions in the obvious sense.

Again, we emphasize that the compatibility condition holds in the point-set category of symmetric spectra rather than in the stable category. We use the term weak equivalence to mean a spectral functor that is a bijection on objects and a weak equivalence (stable equivalence of symmetric spectra) on all mapping spectra. See Definition 5.1 for a more general kind of equivalence.

We have the evident concepts of module and bimodule over spectral categories:

Definition 2.3 Let $\mathcal{C}$ and $\mathcal{D}$ be spectral categories. A left $\mathcal{C}$-module is a spectral functor from $\mathcal{C}$ to symmetric spectra. A right $\mathcal{D}$-module is a spectral functor from $\mathcal{D}^{\text {op }}$ to symmetric spectra. A $(\mathcal{D}, \mathcal{C})$-bimodule is a spectral functor from $\mathcal{D}^{\text {op }} \wedge \mathcal{C}$ to symmetric spectra.

Here $\mathcal{D}^{\text {op }}$ denotes the spectral category with the same objects and mapping spectra as $\mathcal{D}$ but the opposite composition map. The spectral category $\mathcal{D}^{\text {op }} \wedge \mathcal{C}$ has as its objects the cartesian product of the objects,

$$
\operatorname{ob}\left(\mathcal{D}^{\mathrm{op}} \wedge \mathcal{C}\right)=\mathrm{ob} \mathcal{D}^{\mathrm{op}} \times \mathrm{ob} \mathcal{C}
$$

and as its mapping spectra the smash product of the mapping spectra

$$
\left(\mathcal{D}^{\mathrm{op}} \wedge \mathcal{C}\right)\left((d, c),\left(d^{\prime}, c^{\prime}\right)\right)=\mathcal{D}^{o p}\left(d, d^{\prime}\right) \wedge \mathcal{C}\left(c, c^{\prime}\right)
$$

with unit maps the smash product of the unit maps and composition maps the smash product of the composition maps for $\mathcal{D}^{\text {op }}$ and $\mathcal{C}$. Explicitly, a $(\mathcal{D}, \mathcal{C})$-bimodule $\mathcal{M}$ 
consists of a choice of symmetric spectrum $\mathcal{M}(d, c)$ for each $d$ in $\operatorname{ob} \mathcal{D}$ and $c$ in $\operatorname{ob} \mathcal{C}$, together with maps

$$
\mathcal{C}\left(c, c^{\prime}\right) \wedge \mathcal{M}(d, c) \wedge \mathcal{D}\left(d^{\prime}, d\right) \longrightarrow \mathcal{M}\left(d^{\prime}, c^{\prime}\right)
$$

for each $d^{\prime}$ in ob $\mathcal{D}$ and $c^{\prime}$ in ob $\mathcal{C}$, making the obvious unit and associativity diagrams commute. In particular, for any spectral category $\mathcal{C}$, the mapping spectra $\mathcal{C}(-,-)$ define a $(\mathcal{C}, \mathcal{C})$-bimodule. (This example motivates the convention of listing the right module structure first.)

The work of Schwede and Shipley[37] provides the category of $(\mathcal{D}, \mathcal{C})$-bimodules with a closed model structure.

Proposition 2.4 [37, 6.1] The category of $(\mathcal{D}, \mathcal{C})$-bimodules forms a closed model category where the fibrations are the objectwise fibrations and the weak equivalences are the objectwise weak equivalences in the stable model structure on symmetric spectra.

Older $K$-theory literature required "convergence" hypotheses on spectral categories and bimodules, asking for the homotopy groups of the constituent spaces in each mapping spectrum to stabilize. These hypotheses appeared necessary at the time to analyze the homotopy colimits arising in Bökstedt's construction of $T H H$. It was thought that these homotopy colimits could be wrong for a nonconvergent symmetric spectrum because the homotopy groups they computed generally differed from the homotopy groups expected from the underlying prespectrum. Because of [24;39], we now understand that it is the homotopy groups of the underlying prespectrum that may be wrong: The homotopy groups of the prespectrum underlying a symmetric spectrum $X$ do not necessarily agree with the homotopy groups of the object represented by $X$ in the stable category. In general, every symmetric spectrum $X$ admits a weak equivalence $X \rightarrow \tilde{X}$ to a symmetric $\Omega$-spectrum $\tilde{X}$, ie, one whose underlying prespectrum is an $\Omega$-spectrum (level fibrant with adjoint structure maps $\tilde{X}_{n} \rightarrow \Omega \widetilde{X}_{n+1}$ weak equivalences). The correct homotopy groups of $X$ are the homotopy groups of the underlying prespectrum of $\tilde{X}$; when these agree under the comparison map with the homotopy groups of the underlying prespectrum of $X$, then $X$ is said to be semistable. In particular, symmetric $\Omega$-spectra and (more generally) convergent symmetric spectra are semistable. Since we do not include convergence or even semistability hypotheses, for brevity and clarity we adhere to the following convention.

Convention The homotopy groups of a symmetric spectrum $X$ will always mean the homotopy groups of $X$ as an object of the stable category, ie, the abelian groups of maps in the stable category from $S^{q}$ to $X$ (for $q \in \mathbb{Z}$ ), and we will denote these as $\pi_{q} X$. In 
the rare cases when we need to refer to the homotopy groups of the underlying prespectrum of $X$, we will call them the homotopy groups of the underlying prespectrum, and we introduce no notation for these. By weak equivalence of symmetric spectra we shall always mean a weak equivalence in the stable model structure. A weak equivalence is precisely a map that induces an isomorphism on homotopy groups; it does not necessarily induce an isomorphism of the homotopy groups of the underlying prespectra.

Although we do not require convergence hypotheses, they tend to hold for examples of interest. In fact, we can replace an arbitrary small spectral category with a weakly equivalent spectral category that has the same objects but has mapping spectra that are symmetric $\Omega$-spectra. One way of doing this arises from the cofibrantly generated Quillen model category structure on the category of small enriched categories with a fixed set of objects described in [37, Section 6]. The maps in this category are the spectral functors that are the identity on object sets, the fibrations are the maps $\mathcal{C} \rightarrow \mathcal{D}$ that restrict to fibrations of symmetric spectra $\mathcal{C}(x, y) \rightarrow \mathcal{D}(x, y)$ for all $x, y$ and the weak equivalences are the maps that restrict to weak equivalences $\mathcal{C}(x, y) \rightarrow \mathcal{D}(x, y)$ for all $x, y$. We use the following terminology from [37, Section 6].

Definition 2.5 A small spectral category $\mathcal{C}$ is said to be pointwise fibrant if $\mathcal{C}(x, y)$ is a fibrant symmetric spectrum (in the stable model structure) for every pair of objects $x, y$. Likewise, $\mathcal{C}$ is said to be pointwise cofibrant if $\mathcal{C}(x, y)$ is a cofibrant symmetric spectrum for every pair of objects $x, y$. For a spectral functor of small spectral categories $F: \mathcal{C} \rightarrow \mathcal{D}$ that is the identity on the object sets, we say that $F$ is a pointwise weak equivalence or pointwise level equivalence if for every pair of objects $x, y$, the map $F: \mathcal{C}(x, y) \rightarrow \mathcal{D}(x, y)$ is a weak equivalence or level equivalence, respectively, of symmetric spectra.

Fibrant replacement in the model structures of [37, Section 6] then gives most of the following proposition. The rest follows from the easy observation that the factorization functors constructed by the small objects argument on the category of small spectral categories with a fixed set of objects still behave well with respect to spectral functors that are not the identity on object sets.

Proposition 2.6 [37, 6.3] Given a small spectral category $\mathcal{C}$, there exists a small spectral category $\mathcal{C}^{\Omega}$ and a spectral functor $R: \mathcal{C} \rightarrow \mathcal{C}^{\Omega}$ such that

(i) $\mathcal{C}^{\Omega}$ has the same objects as $\mathcal{C}$ and $R$ is the identity map on objects,

(ii) $\mathcal{C}^{\Omega}$ is pointwise fibrant, and

(iii) $R$ is a pointwise weak equivalence.

Moreover, $(-)^{\Omega}$ and $R$ may be constructed as an endofunctor and natural transformation on the category of small spectral categories. 
Applying cofibrant replacement in the model structure of [37, Section 6], we obtain the following complementary proposition.

Proposition $2.7[37,6.3]$ Given a small spectral category $\mathcal{C}$, there exists a small spectral category $\mathcal{C}^{\text {Cell }}$ and a spectral functor $Q: \mathcal{C}^{\text {Cell }} \rightarrow \mathcal{C}$ such that

(i) $\mathcal{C}^{\text {Cell }}$ has the same objects as $\mathcal{C}$ and $Q$ is the identity map on objects,

(ii) $\mathcal{C}^{\text {Cell }}(x, y)$ is pointwise cofibrant, and

(iii) $Q$ is a pointwise level equivalence.

Moreover, (-) Cell and $Q$ may be constructed as an endofunctor and natural transformation on the category of small spectral categories.

We also use an analogous proposition in the setting of bimodules.

Proposition 2.8 Assume that $\mathcal{C}$ and $\mathcal{D}$ are pointwise cofibrant small spectral categories. If $\mathcal{M}$ is a cofibrant $(\mathcal{D}, \mathcal{C})$-bimodule, then $\mathcal{M}$ is objectwise cofibrant, ie, $\mathcal{M}(d, c)$ is a cofibrant symmetric spectrum for every $(d, c)$ in $\mathcal{D}^{\text {op }} \wedge \mathcal{C}$.

In addition to providing the formal technical results above, the model theory of enriched categories also explains the relationship of small spectral categories to small DG-categories. Sharp statements involve categories enriched over $H \mathbb{Z}$-modules (in symmetric spectra of simplicial sets) or Quillen equivalently, categories enriched over symmetric spectra of simplicial abelian groups. For brevity, we will call these $H \mathbb{Z}$-categories and Ab-spectral categories, respectively. Note that the category of $H \mathbb{Z}$-modules is symmetric monoidal under $\wedge_{H \mathbb{Z}}$ and its derived category is symmetric monoidally equivalent to the derived category of $\mathbb{Z}$ (in particular, $\wedge_{H \mathbb{Z}}$ is more like $\otimes_{\mathbb{Z}}$ than like $\wedge$ ). Shipley [40, Section 2.2] produces a zigzag of "weak monoidal Quillen equivalences" relating $H \mathbb{Z}$-modules to symmetric spectra of simplicial abelian groups to symmetric spectra of nonnegatively graded and integer graded chain complexes to chain complexes. A slight modification gives a comparison for algebras or more generally categories with a fixed object set $O$ : Proposition 6.4 of [37] (or [12, A.3]) gives a zigzag of Quillen equivalences between the model categories of DG-categories with object set $O, \mathrm{Ab}$-spectral categories with object set $O$, and $H \mathbb{Z}$-categories with object set $O$.

Definition 2.9 Given a small DG-category, the associated Ab-spectral category model or associated $H \mathbb{Z}$-category model is the Ab-spectral category or $H \mathbb{Z}$-category (respectively) with the same object set constructed from the zigzag of Quillen equivalences outlined above using the fibrant or cofibrant replacement functor (as needed) at every stage. 
By neglect of structure, an Ab-spectral category or $H \mathbb{Z}$-category is in particular a spectral category. We then get the associated spectral category model from the associated $\mathrm{Ab}$-spectral or $H \mathbb{Z}$-category model. Because the cofibrant and fibrant replacement functors in spectral categories with fixed object sets also behave well with respect to spectral functors that are not the identity on object sets, the construction of associated spectral categories in fact produces a functor from small DG-categories to small spectral categories.

Proposition 2.10 The zigzags of Quillen equivalences and cofibrant/fibrant replacement functors above assemble into a functor from the category of small DG-categories to the category of small spectral categories.

Note that we do not assert that the associated spectral category functor provides a 2 -functor; it does not preserve composition of natural transformations. Using the usual reformulation of natural transformations and composites of natural transformations as spectral functors from related spectral categories, we do see that the associated spectral category functor preserves natural transformations and their compositions in some coherent homotopy sense. Since we do not need this theory here, we leave a rigorous formulation to the interested reader.

\section{Review of $T H H, T R$ and $T C$}

In this section, we review the definition of $T H H, T R$, and $T C$ of small spectral categories. We begin with a review of the cyclic bar construction for small spectral categories and the variant defined by Bökstedt [3] and Dundas and McCarthy [13] necessary for the construction of $T C$. We finish with a brief review of the definition of cyclotomic spectra and the construction of $T R$ and $T C$.

The following cyclic bar construction gives the "topological" analogue of the Hochschild-Mitchell complex.

Definition 3.1 For a small spectral category $\mathcal{C}$ and $(\mathcal{C}, \mathcal{C})$-bimodule $\mathcal{M}$, let

$$
N_{q}^{\mathrm{cy}}(\mathcal{C} ; \mathcal{M})=\bigvee \mathcal{C}\left(c_{q-1}, c_{q}\right) \wedge \cdots \wedge \mathcal{C}\left(c_{0}, c_{1}\right) \wedge \mathcal{M}\left(c_{q}, c_{0}\right)
$$

where the sum is over the $(q+1)$-tuples $\left(c_{0}, \ldots, c_{q}\right)$ of objects of $\mathcal{C}$. This becomes a simplicial object in symmetric spectra using the usual cyclic bar construction face and degeneracy maps: The unit maps of $\mathcal{C}$ induce the degeneracy maps, and the two action maps on $\mathcal{M}$ (for $d_{0}$ and $d_{q}$ ) and the composition maps in $\mathcal{C}$ (for $d_{1}, \ldots, d_{q-1}$ ) induce the face maps. We denote the diagonal (geometric realization) symmetric spectrum as $N^{\text {cy }}(\mathcal{C} ; \mathcal{M})$ and write $N^{\text {cy }}(\mathcal{C})$ for $N^{\text {cy }}(\mathcal{C} ; \mathcal{C})$. 
The previous construction turns out to be slightly inconvenient to use as the definition of the topological Hochschild homology of a small spectral category. This construction typically only has the correct homotopy type when the smash products that comprise the terms of the sum represent the derived smash product. The analogous problem arises in the context of Hochschild homology of DG-categories, where the tensor product may fail to have the right quasi-isomorphism type when the mapping complexes are not DG-flat. Just as in that context, this problem can be overcome using resolutions, such as the ones in Propositions 2.7 and 2.8. There is a further more subtle difficulty with this construction, however. While $N^{\mathrm{cy}}(\mathcal{C})$ obtains an $S^{1}$-action by virtue of being the geometric realization of a cyclic complex, the resulting equivariant spectrum does not have the necessary additional structure to define $T C$ (a well-known problem with this kind of cyclic bar construction definition of $T H H$ in modern categories of spectra). The correct definition, due to Bökstedt [3] for symmetric ring spectra and generalized by Dundas and McCarthy [13] to small spectral categories, does not suffer from either of these deficiencies.

We give a revisionist explanation of the Bökstedt-Dundas-McCarthy construction, taking advantage of later results of Shipley [39] on the derived smash product of symmetric spectra. Let $\mathcal{I}$ be the category with objects the finite sets $\mathbf{n}=\{1, \ldots, n\}$ (including $\mathbf{0}=\{\}$ ), and with morphisms the injective maps. For a symmetric spectrum $A$, write $A_{n}$ for the $n$-th space. The association $\mathbf{n} \mapsto \Omega^{n}\left|A_{n}\right|$ extends to a functor from $\mathcal{I}$ to spaces, where $|-|$ denotes geometric realization. More generally, given symmetric spectra $A^{0}, \ldots, A^{q}$ and a space $X$, we obtain a functor from $\mathcal{I}^{q+1}$ to spaces that sends $\overrightarrow{\mathbf{n}}=\left(\mathbf{n}_{0}, \ldots, \mathbf{n}_{q}\right)$ to

$$
\Omega^{n_{0}+\cdots+n_{q}}\left(\left|A_{n_{q}}^{q} \wedge \cdots \wedge A_{n_{0}}^{0}\right| \wedge X\right),
$$

which is also natural in $X$. Restricting to the case when $X$ is a sphere $S^{n}$, we form this into a symmetric spectrum following [39] (but using different notation).

Definition 3.2 $[39,4.2 .1]$ Let $D\left(A^{q}, \ldots, A^{0}\right)$ be the symmetric spectrum (of topological spaces) with $n$-th space

$$
D_{n}\left(A^{q}, \ldots, A^{0}\right)=\operatorname{hocolim}_{\overrightarrow{\mathbf{n}} \in \mathcal{I}^{q+1}} \Omega^{n_{0}+\cdots+n_{q}}\left(\left|A_{n_{q}}^{q} \wedge \cdots \wedge A_{n_{0}}^{0}\right| \wedge S^{n}\right),
$$

and the evident structure maps.

The following is the main lemma of [39].

Proposition 3.3 $[39,4.2 .3] \quad D\left(A^{q}, \ldots, A^{0}\right)$ is canonically isomorphic in the stable category to the derived smash product of the $A^{i}$. 
This motivates the following definition.

Definition 3.4 Given a small spectral category $\mathcal{C}$, a $(\mathcal{C}, \mathcal{C})$-bimodule $\mathcal{M}$ and a space $X$, let $\mathcal{G}(\mathcal{C} ; \mathcal{M} ; X)_{\overrightarrow{\mathbf{n}}}$ be the functor from $\mathcal{I}^{q+1}$ to spaces defined on $\overrightarrow{\mathbf{n}}=$ $\left(\mathbf{n}_{0}, \ldots, \mathbf{n}_{q}\right)$ by

$\mathcal{G}(\mathcal{C} ; \mathcal{M} ; X)_{\overrightarrow{\mathbf{n}}}=\Omega^{n_{0}+\cdots+n_{q}}\left(\bigvee\left|\mathcal{C}\left(c_{q-1}, c_{q}\right)_{n_{q}} \wedge \cdots \wedge \mathcal{C}\left(c_{0}, c_{1}\right)_{n_{1}} \wedge \mathcal{M}\left(c_{q}, c_{0}\right)_{n_{0}}\right| \wedge X\right)$, and let

$$
\operatorname{THH}_{q}(\mathcal{C} ; \mathcal{M})(X)=\operatorname{hocolim}_{\overrightarrow{\mathbf{n}} \in \mathcal{I}^{q+1}} \mathcal{G}(\mathcal{C} ; \mathcal{M} ; X)_{\overrightarrow{\mathbf{n}}} .
$$

This assembles into a simplicial space, functorially in $X$, as follows. The degeneracy maps are induced by the unit maps $S^{0} \rightarrow \mathcal{C}\left(c_{i}, c_{i}\right)_{0}$ and the functor

$$
\left(\mathbf{n}_{0}, \ldots, \mathbf{n}_{q}\right) \mapsto\left(\mathbf{n}_{0}, \ldots, \mathbf{0}, \ldots, \mathbf{n}_{q}\right)
$$

from $I^{q+1}$ to $I^{q+2}$. The face maps are induced by the two action maps on $\mathcal{M}$ (for $d_{0}$ and $d_{q}$ ) and the composition maps in $\mathcal{C}$ (for $d_{1}, \ldots, d_{q-1}$ ) together with a functor $\mathcal{I}^{q+1} \rightarrow \mathcal{I}^{q}$ induced by the appropriate disjoint union isomorphism $\left(\mathbf{n}_{i}, \mathbf{n}_{i+1}\right) \mapsto \mathbf{n}$ or $\left(\mathbf{n}_{q}, \mathbf{n}_{0}\right) \mapsto \mathbf{n}$ for $n=n_{i}+n_{i+1}$ or $n=n_{q}+n_{0}$. We write $\operatorname{THH}(\mathcal{C} ; \mathcal{M})(X)$ for the geometric realization.

$\operatorname{THH}(\mathcal{C} ; \mathcal{M})(X)$ is a continuous functor in the variable $X$, and so by restriction to the spheres $S^{n}$ specifies a symmetric spectrum which we denote $T H H(\mathcal{C} ; \mathcal{M})$ or $T H H(C)$ for $\mathcal{M}=\mathcal{C}$. The fact that the symmetric spectrum $T H H$ is the restriction of a continuous functor implies that it is semistable [32,8.7] and so the object that it represents in the stable category agrees with its underlying prespectrum. With additional hypotheses of "convergence" and "connectivity", $T H H$ is often an $\Omega$-spectrum; see, for example, of Hesselholt and Madsen [20, Proposition 2.4].

The following propositions, which are essentially the "many objects" versions of [39, 4.2.8-9] and an easy consequence of the theory developed in [39], show that in the stable category $\operatorname{THH}(\mathcal{C})$ is simply a homotopically well-behaved model of the Hochschild-Mitchell complex.

Proposition 3.5 There is a natural map in the stable category from $\operatorname{THH}(\mathcal{C} ; \mathcal{M})$ to $N^{\text {cy }}(\mathcal{C} ; \mathcal{M})$ that is an isomorphism when $\mathcal{C}$ is pointwise cofibrant.

Proposition 3.6 Let $F: \mathcal{C} \rightarrow \mathcal{C}^{\prime}$ be a weak equivalence of small spectral categories, $\mathcal{M}^{\prime}$ a $\left(\mathcal{C}^{\prime}, \mathcal{C}^{\prime}\right)$-bimodule, $F^{*} \mathcal{M}^{\prime}$ the $(\mathcal{C}, \mathcal{C})$-bimodule obtained by restriction of scalars, and $\mathcal{M} \rightarrow F^{*} \mathcal{M}^{\prime}$ a weak equivalence of $(\mathcal{C}, \mathcal{C})$-bimodules. Then the induced map $\operatorname{THH}(\mathcal{C} ; \mathcal{M}) \rightarrow \operatorname{THH}\left(\mathcal{C}^{\prime} ; \mathcal{M}^{\prime}\right)$ is a weak equivalence. 
As a consequence of the previous propositions, $\operatorname{THH}(\mathcal{C} ; \mathcal{M})$, and $\operatorname{THH}(\mathcal{C})$ always have the correct homotopy type even when $N^{\text {cy }}(\mathcal{C} ; \mathcal{M})$ or $N^{\text {cy }}(\mathcal{C})$ does not. We also note that Proposition 3.5 does not require the bimodule $\mathcal{M}$ to be objectwise cofibrant.

We now list the usual bimodule properties of $T H H$ that we require in this paper. Proofs of these properties appear in the literature [13] under more restrictive hypotheses (ie, connectivity and convergence).

Proposition 3.7 Let $\mathcal{C}$ be a small spectral category.

(i) A weak equivalence of $(\mathcal{C}, \mathcal{C})$-bimodules $\mathcal{M} \rightarrow \mathcal{M}^{\prime}$ induces a weak equivalence $\operatorname{THH}(\mathcal{C} ; \mathcal{M}) \rightarrow \operatorname{THH}\left(\mathcal{C} ; \mathcal{M}^{\prime}\right)$.

(ii) A cofibration sequence of $(\mathcal{C}, \mathcal{C})$-bimodules $\mathcal{M} \rightarrow \mathcal{M}^{\prime} \rightarrow \mathcal{M}^{\prime \prime} \rightarrow \Sigma \mathcal{M}$ induces a homotopy cofiber sequence on $\mathrm{THH}$.

(iii) A fibration sequence of level fibrant $(\mathcal{C}, \mathcal{C})$-bimodules $\Omega \mathcal{M}^{\prime \prime} \rightarrow \mathcal{M} \rightarrow \mathcal{M}^{\prime} \rightarrow \mathcal{M}^{\prime \prime}$ induces a homotopy fibration sequence on $\mathrm{THH}$.

Proof The first statement is a special case of Proposition 3.6. For the second statement, we can identify $T H H_{\bullet}$ levelwise as the homotopy colimit (over $\mathcal{I}^{\bullet+1}$ ) of the symmetric spectra $\mathcal{G}\left(\mathcal{C} ; \mathcal{M} ; S^{(-)}\right)_{\overrightarrow{\mathbf{n}}}$. The second statement now follows from the observation that $\mathcal{G}$ preserves homotopy cofibration sequences in the $\mathcal{M}$ variable and that homotopy colimits and geometric realization preserve homotopy cofibration sequences. The third statement follows from the second since homotopy fibration sequences and homotopy cofibration sequences agree up to sign.

We now give a minimal review of the definition of $T R$ and $T C$; we refer the reader interested in more details to the excellent discussions of $T R$ and $T C$ by Hesselholt and Madsen [20;21]. For an $S^{1}$-space $X$, the space $T H H(\mathcal{C})(X)$ has two $S^{1}$ actions, one coming from $X$ and the other coming from the cyclic structure. Using the diagonal action and restricting to representation spheres $S^{V}$ makes $T H H(\mathcal{C})(-)$ into an equivariant orthogonal spectrum [32, Section II.2]; however, $\operatorname{THH}(\mathcal{C})$ has even more structure, that of a cyclotomic spectrum [21, Section 1.1], [20, Definition 2.2]. We review the definition of a cyclotomic spectrum in detail in the next section, but in brief the structure on $\mathrm{THH}$ derives from the fundamental fixed point map

$$
(\operatorname{THH}(\mathcal{C})(X))^{H} \longrightarrow T H H(\mathcal{C})\left(X^{H}\right)
$$

for $S^{1}$-spaces $X$ and finite subgroups $H$ of $S^{1}$. This induces maps in the equivariant stable category

$$
r_{H}: \rho_{H}^{\#} \Phi^{H} T H H(\mathcal{C}) \longrightarrow T H H(\mathcal{C})
$$


that are nonequivariant weak equivalences. Here $\Phi^{H}$ denotes the (derived) geometric fixed point spectrum, and when $H$ is the subgroup with $n$ elements, $\rho_{H}$ is the $n$-th root isomorphism $S^{1} \cong S^{1} / H ; \rho_{H}^{\#}$ converts the $S^{1} / H$-spectrum $\Phi^{H} T H H(\mathcal{C})$ back to an $S^{1}$-spectrum via the isomorphism $\rho$. Essentially, a cyclotomic spectrum consists of an $S^{1}$-equivariant spectrum indexed on a complete universe together with weak equivalences $r_{H}$ of the form above, called cyclotomic structure maps, satisfying certain coherence properties [20, Definition 2.2; 21, Section 1.1]. We give a precise formulation of the point-set category of cyclotomic spectra we use here in Definition 4.2 in the next section; for now, the only detail we need is that $T H H$ defines a functor from small spectral categories to the point-set category of cyclotomic spectra (Theorem 4.9).

For a fixed prime $p$ and each $n$, let $C_{p^{n}} \subset S^{1}$ denote the cyclic subgroup of order $p^{n}$. We then have maps in the (nonequivariant) stable category

$$
F, R: T H H(C){ }^{C_{p}^{n}} \longrightarrow T H H(C){ }^{C_{p^{n-1}}},
$$

where $F$ is the inclusion of the fixed points and $R$ is the map induced by the composite of the map from the fixed point spectrum to the geometric fixed point spectrum $T H H(\mathcal{C})^{C_{p}} \rightarrow \Phi^{C_{p}} T H H(\mathcal{C})$ and the cyclotomic structure map $r_{C_{p}}: \Phi^{C_{p}} T H H(\mathcal{C}) \rightarrow$ $T H H(C)$; see [21, Section 1.1; 20, Section 2.2] or Section 4 below. We need functorial point-set versions of these maps to construct $T C$ as a functor on small spectral categories. In [21], the connectivity and convergence hypotheses used there imply that $T H H(\mathcal{C})$ is an equivariant $\Omega$-spectrum relative to the family of finite subsets of $S^{1}$; the point-set maps $F, R$ in [21] are then constructed using the point-set fixed point spectra as models for the derived fixed point spectra. In our context, we need to use an $\Omega$-spectrum replacement functor in the category of cyclotomic spectra; see Definition 4.5 and Theorem 4.7 in the next section. For such a functor $Q$, we get appropriate point-set maps

$$
F, R: Q(T)^{C_{p^{n}}} \longrightarrow Q(T)^{C_{p^{n-1}}} .
$$

which are functorial in the cyclotomic spectrum $T$.

Definition 3.8 Let $Q$ be an $\Omega$-spectrum replacement functor in the category of cyclotomic spectra and write $T(\mathcal{C})$ for $Q(T H H(\mathcal{C}))$. Then $T R^{\bullet}(\mathcal{C})$ is the pro-spectrum $\left\{T(\mathcal{C})^{C_{p^{n}}}\right\}$ under the maps $R$, and $T R(\mathcal{C})$ is the homotopy limit. $T C(\mathcal{C})$ and $T C^{\bullet}(\mathcal{C})$ are the spectrum and pro-spectrum obtained from $T R(\mathcal{C})$ and $T R^{\bullet}(\mathcal{C})$ as the homotopy equalizer of the maps $F$ and $R$.

Note that a map in the $S^{1}$-equivariant stable category induces a (nonequivariant) weak equivalence on fixed point spectra for all finite subgroups of $S^{1}$ if and only if it induces a (nonequivariant) weak equivalence on geometric fixed point spectra for all 
finite subgroups [34, XVI.6.4]. It follows that a cyclotomic map of cyclotomic spectra induces a weak equivalence of fixed point spectra for all finite subgroups of $S^{1}$ if and only if it is a nonequivariant weak equivalence. In particular, we obtain the following proposition.

Proposition 3.9 A spectral functor of small spectral categories $\mathcal{C} \rightarrow \mathcal{D}$ that induces a weak equivalence on THH induces a weak equivalence on TR and TC.

Likewise, using the same principle on the cofiber of a map of cyclotomic spectra, we obtain the following proposition. Applying this proposition in examples when $T H H(C)$ is contractible, localization cofibration sequences on $T R$ and $T C$ follow from ones on $\mathrm{THH}$.

Proposition 3.10 For a strictly commuting square of small spectral categories

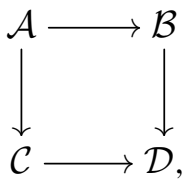

if the induced square on THH is homotopy cocartesian, then so are the induced squares on $T R$ and $T C$.

Finally, we turn to DG-categories. For a DG-category $\mathcal{C}^{\mathrm{DG}}$, we can consider $T H H$ of the associated spectral category $\mathcal{C}^{S}$. Propositions 2.10 and 3.9 show that defining $T H H, T R$ and $T C$ of $\mathcal{C}^{\text {DG }}$ in terms of $T H H\left(\mathcal{C}^{S}\right)(-)$ constructs $T H H, T R$ and $T C$ as functors from the category of DG-categories and DG-functors to the stable category; this is Theorem 1.2.

\section{Details of the category of cyclotomic spectra}

Starting in Section 7, we will want a construction of $T H H$ that provides a pointset functor from the category of small spectral categories to a point-set category of cyclotomic spectra. We describe such a category and functor in this section.

In contrast to the canonical definition of cyclotomic spectra in [20] (which is in terms of Lewis-May spectra), we use orthogonal spectra. To make this section easier to compare with the definition in [20], we follow the notation there as much as possible (but with some abbreviation). The following summarizes the notation and terminology regarding the circle group, its subgroups, and its representations. 
Notation 4.1 Let $G$ denote the circle group of unit complex numbers. For $n=$ $1,2,3, \ldots$, let $C_{n}$ (or just $C$ ) denotes the subgroup with $n$ elements. Write $\rho_{n}$ for the $n$-th root isomorphism $G \rightarrow G / C_{n}$ and for a $G / C_{n}$-space $X$ (eg, $X=Y^{C_{n}}$ for some $G$-space $Y$ ), write $\rho_{n}^{*} X$ for the $G$-space obtained via this isomorphism. Throughout this section orthogonal $G$-representation will mean a finite dimensional real $G$-inner product space. Let $\mathbb{C}(0)=\mathbb{C}$ denote the complex numbers with trivial $G$-action, $\mathbb{C}(1)$ the complex numbers with the standard $G$-action, and $\mathbb{C}(n)=\mathbb{C}(1) \otimes_{\mathbb{C}} \cdots \otimes_{\mathbb{C}} \mathbb{C}(1)$ ( $n$-factors). Let

$$
\mathcal{U}=\bigoplus_{n=0}^{\infty} \bigoplus_{r=1}^{\infty} \mathbb{C}(n)
$$

(a direct sum of infinitely many copies of each $\mathbb{C}(n)$ ) with the standard inner product; this is a complete $G$-universe, a real countable-dimensional $G$-inner product space containing an isomorphic copy of every orthogonal $G$-representation. When we write $V<\mathcal{U}$, we will always understand $V$ to be a finite dimensional $G$-invariant vector subspace (and thus, an orthogonal $G$-representation). For $V<W<\mathcal{U}$, let $W-V$ denote the orthogonal complement of $V$ in $W$.

We now give a very brief review of the definition of orthogonal $G$-spectra; see Mandell and May [31, Section II.2,II.4] for a complete treatment. For orthogonal $G$-representations $V$ and $W$, let $\mathscr{F}_{G}(V, W)$ denote the Thom space of the orthogonal complement $G$-bundle of (nonequivariant) linear isometries from $V$ to $W$ [31, II.4.1] (an element of the complement $G$-bundle consists of a linear isometry $V \rightarrow W$ and a point in the orthogonal complement of the image). Composition of isometries and addition in the codomain vector space induces composition maps

$$
\mathscr{\mathscr { F }}_{G}(W, Z) \wedge \mathscr{\mathscr { F }}_{G}(V, W) \longrightarrow \mathscr{\mathscr { F }}_{G}(V, Z),
$$

which together with the obvious identity elements make $\mathscr{f}_{G}$ a category enriched in based $G$-spaces (with objects the orthogonal $G$-representations). An orthogonal $G$-spectrum is a $\mathscr{F}_{G}$-space [31, II.4.3], an enriched functor from (a skeleton of) $\mathscr{I}_{G}$ to based $G-$ spaces. That is, an orthogonal $G$-spectrum $T$ consists of a based $G$-space $T(V)$ for each orthogonal $G$-representation $V$ together with based $G$-maps

$$
\mathscr{F}_{G}(V, W) \wedge T(V) \longrightarrow T(W)
$$

satisfying the obvious unit and associativity properties. We recall that the homotopy groups of $T$ are defined by

$$
\pi_{q}^{H} T= \begin{cases}\underset{V<\mathcal{U}}{\operatorname{colim}} \pi_{q}\left(\left(\Omega^{V} T(V)\right)^{H}\right) & q \geq 0, \\ \mathbb{C}^{-q}<\operatorname{colim}_{<} \pi_{-q}\left(\left(\Omega^{V-\mathbb{C}^{-q}} T(V)\right)^{H}\right) & q<0,\end{cases}
$$


for $q \in \mathbb{Z}$ and $H$ a closed subgroup of $G$ [31, III.3.2]. We also have a similar formula for the homotopy groups of the geometric fixed point spectrum of the underlying object in the equivariant stable category; these groups were denoted as $\rho_{q}^{H}$ in [31, Section V.4], but we will denote them as $\pi_{q}^{\Phi H}$ to avoid confusion with the isomorphisms $\rho_{n}$ in Notation 4.1. Then

$$
\pi_{q}^{\Phi H} T= \begin{cases}\underset{V<\mathcal{U}}{\operatorname{colim}} \pi_{q}\left(\Omega^{V^{H}}\left(T(V)^{H}\right)\right) & q \geq 0, \\ \underset{\mathbb{C}^{-q}<V<\mathcal{U}}{\operatorname{colim}} \pi_{-q}\left(\Omega^{V^{H}-\mathbb{C}^{-q}}\left(T(V)^{H}\right)\right) & q<0,\end{cases}
$$

for $q \in \mathbb{Z}$ and $H$ a closed subgroup of $G$ [31, V.4.8.(iii),V.4.12].

Throughout this paper, we use the following precise definition for cyclotomic spectra.

Definition 4.2 A cyclotomic spectrum is an orthogonal $G$-spectrum $T$ together with $G$-equivariant maps

$$
r_{n, V}: \rho_{n}^{*}\left(T(V)^{C_{n}}\right) \longrightarrow T\left(\rho_{n}^{*}\left(V^{C_{n}}\right)\right)
$$

for $V$ an orthogonal $G$-representation and $n=1,2,3, \ldots$, satisfying the following conditions.

(i) $r_{1, V}$ is the identity for all $V$.

(ii) For any $V, W, n$, the diagram

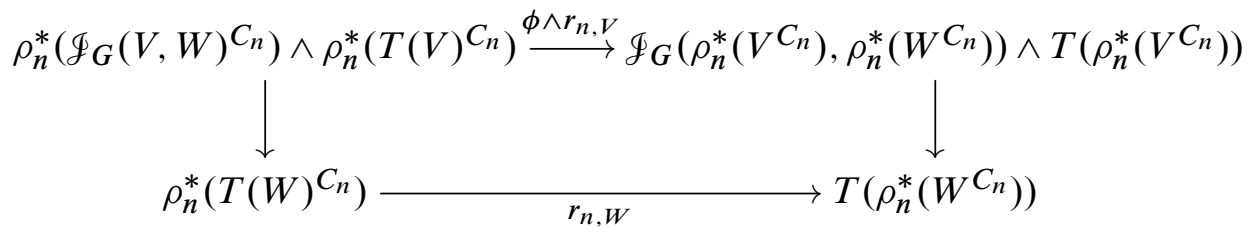

commutes. Here vertical maps are induced by the $\mathscr{f}_{G}$-space structure maps. In the top horizontal map, the map

$\phi: \rho_{n}^{*}\left(\mathscr{F}_{G}(V, W)^{C_{n}}\right) \longrightarrow \rho_{n}^{*} \mathscr{F}_{G / C_{n}}\left(V^{C_{n}}, W^{C_{n}}\right)=\mathscr{F}_{G}\left(\rho_{n}^{*}\left(V^{C_{n}}\right), \rho_{n}^{*}\left(W^{C_{n}}\right)\right)$

is induced by the fixed point functor $\phi: \mathscr{I}_{G}(V, W)^{C_{n}} \rightarrow \mathscr{I}_{G / C_{n}}\left(V^{C_{n}}, W^{C_{n}}\right)$ described in [32, Section V.4].

(iii) For all $V, m, n$, the diagram

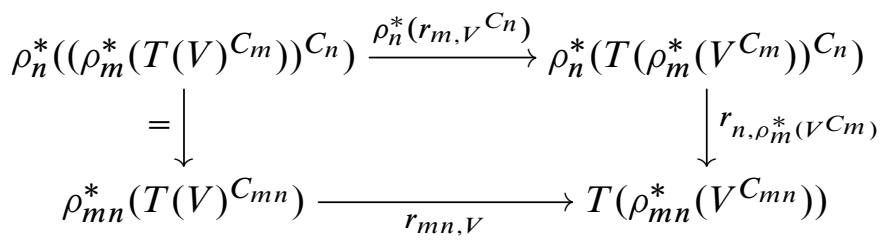

commutes (using $\rho_{m n}^{*}\left(X^{C_{m n}}\right)=\rho_{n}^{*}\left(\left(\rho_{m}^{*}\left(X^{C_{m}}\right)\right)^{C_{n}}\right)$ for any $G$-space $\left.X\right)$. 
(iv) For all $q \in \mathbb{Z}$ and all $n$, the map

$$
\begin{aligned}
& \underset{V<\mathcal{U}}{\operatorname{colim}} \pi_{q} \Omega^{V^{C n}}\left(T(V)^{C_{n}}\right) \longrightarrow \underset{W<\mathcal{U}^{C n}}{\operatorname{colim}} \pi_{q} \Omega^{W}\left(T\left(\rho_{n}^{*} W\right)\right) \quad \text { if } q \geq 0, \\
& \underset{\mathbb{C}^{-q}<V<\mathcal{U}}{\operatorname{colim}} \pi_{-q} \Omega^{V^{C n}-\mathbb{C}^{-q}}\left(T(V)^{C_{n}}\right) \longrightarrow \underset{\mathbb{C}^{-q}<W<\mathcal{U} C_{n}}{\operatorname{colim}} \pi_{-q} \Omega^{W-\mathbb{C}^{-q}} T\left(\rho_{n}^{*} W\right) \text { if } q<0
\end{aligned}
$$

is an isomorphism.

A map of cyclotomic spectra $(T, r) \rightarrow\left(T^{\prime}, r^{\prime}\right)$ is a map of orthogonal spectra $f: T \rightarrow T^{\prime}$ making the diagram

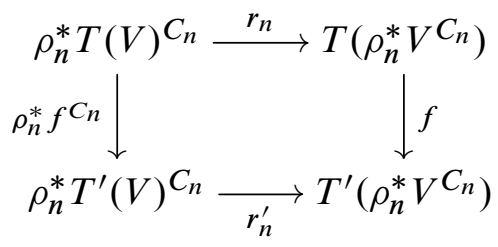

commute for all $n, V$.

The explanation of (iv) is that the colimit on the left is $\pi_{q}^{\Phi C_{n}} T=\pi_{q} \Phi^{C_{n}} T$ and the colimit on the right is $\pi_{q} T$. Condition (iv) then insures that the induced map in the stable category $\Phi^{C_{n}} T \rightarrow T$ constructed as in [20, Lemma 2.2] is an isomorphism. We can rephrase the previous definition in terms of the point-set model Fix ${ }^{C_{n}} T$ for the geometric fixed point spectrum $\Phi^{C_{n}} T$ from [31, Section V.4].

Remark 4.3 In [31, Section V.4], a version of the geometric fixed point spectrum called Fix ${ }^{C_{n}} T$ is constructed as a $\mathscr{F}_{E_{n}}$-space (for $E_{n}$ the extension $C_{n} \succ G \rightarrow G / C_{n}$ ), where $\mathscr{F}_{E_{n}}=\left(\mathscr{F}_{G}\right)^{C_{n}}$. We write $i_{n}$ for the inclusion of $\mathscr{F}_{E_{n}}$ into $\mathscr{F}_{G}$ and $\phi_{n}: \mathscr{F}_{E_{n}} \rightarrow$ $\mathscr{I}_{G / C_{n}}$ for the functor that sends $V$ to $V^{C_{n}}$; then $i_{n}$ is an enriched functor of categories enriched in based $G$-sets and $\phi_{n}$ is an enriched functor of categories enriched in based $G / C_{n}$-sets. The functor $\phi_{n}$ allows us to regard an orthogonal $G / C_{n}$-spectrum as a $\mathscr{F}_{E_{n}}$-space, and we will use $\rho_{n}^{*} \phi_{n}$ to regard a $\rho_{n}^{*} \mathscr{F}_{G / C_{n}}$-space as a $\rho_{n}^{*} \mathscr{F}_{E_{n}}$-space. Of course, $\rho_{n}$ induces an isomorphism of enriched categories $\rho_{n}^{*} \mathscr{F}_{G / C_{n}} \rightarrow \mathscr{F}_{G}$, and we write $\phi_{n}^{!}$for the composite enriched functor $\rho_{n}^{*} \mathscr{F}_{E_{n}} \rightarrow \mathscr{F}_{G}$. Recall that Fix ${ }^{C_{n}} T$ is the functor $\left(T \circ i_{n}\right)^{C_{n}}$. Definition 4.2 is equivalent to asking for a map of $\rho_{n}^{*} \mathscr{F}_{E_{n}}$-spaces

$$
r_{n}: \rho_{n}^{*} \operatorname{Fix}^{C_{n}} T \longrightarrow T \circ \phi_{n}^{!}
$$


for each $n$ that is a nonequivariant weak equivalence, is the identity for $n=1$, and that makes the following diagram of $\rho_{m n}^{*} \mathscr{F}_{E_{m n}}$-spaces commute for all $m n$ :

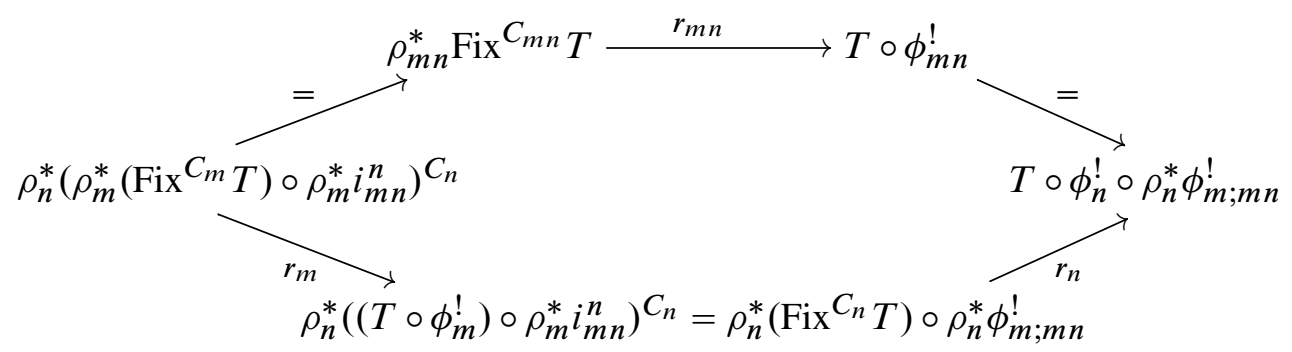

Here the maps labelled $r_{m}$ and $r_{n}$ are really the maps induced by $r_{m}$ and $r_{n}$ inside the fixed points and compositions. The functor labelled $i_{m n}^{n}$ is the inclusion of $\mathscr{F}_{E_{m n}}=$ $\mathscr{F}_{G}^{C_{m n}}$ into $\mathscr{F}_{E_{m}}=\mathscr{F}_{G}^{C_{m}}$ and the functor labelled $\phi_{m ; m n}^{!}$is the map $\rho_{m}^{*} \mathscr{F}_{E_{m n}} \rightarrow \mathscr{F}_{E_{n}}$ obtained from the restriction of $\phi_{m}^{!}$. The functor $\rho_{n}^{*} \phi_{m ; m n}^{!}$is then the induced functor

$$
\rho_{n}^{*}\left(\rho_{m}^{*} \mathscr{F}_{E_{m n}}\right) \longrightarrow \rho_{n}^{*} \mathscr{F}_{E_{n}} .
$$

The diagonal equality on the left arises from the equation

$$
i_{m n}=i_{m} \circ i_{m n}^{n},
$$

the horizontal equality on the bottom arises from the equation

$$
\phi_{m}^{!} \circ \rho_{m}^{*} i_{m n}^{n}=i_{n} \circ \phi_{m ; m n}^{!},
$$

and the diagonal equality on the right arises from the equation

$$
\phi_{m n}^{!}=\phi_{n}^{!} \circ \rho_{n}^{*} \phi_{m ; m n}^{!}
$$

In this case, the spacewise definition above seems less complicated than the equivalent diagram space definition here.

Although we cannot expect the category of cyclotomic spectra to admit general limits and colimits (because of condition (iv)), it is closed under homotopy colimits and finite homotopy limits in orthogonal spectra.

Proposition 4.4 If $\Psi$ is a functor from a small category $\mathcal{D}$ to cyclotomic spectra, then the homotopy colimit of $\Psi$ in orthogonal $G$-spectra admits the natural structure of a cyclotomic spectrum. If $\mathcal{D}$ has only finitely many objects and finitely many sequences of composable nonidentity morphisms, then the homotopy limit of $\Psi$ in orthogonal $G$-spectra likewise admits the natural structure of a cyclotomic spectrum. 
Proof Under the hypotheses, we can compute the homotopy colimit and homotopy limit spacewise using the usual bar and cobar constructions, respectively. The structure maps $r_{n, V}$ then commute with these constructions and conditions (i)-(iii) in the definition of cyclotomic spectra are clearly preserved. The standard properties of homotopy groups and geometric fixed points in the equivariant stable category imply that condition (iv) is also preserved.

In this section, we write $T^{C_{n}}$ for the point-set fixed point orthogonal spectrum of an orthogonal $G$-spectrum $T$ : It is a nonequivariant orthogonal spectrum with $T^{C_{n}}(V)=$ $(T(V))^{C_{n}}$ where we regard a (nonequivariant) inner product space $V$ as a $G$-inner product space with trivial $G$-action. The inclusion of $C_{m}$ in $C_{m n}$ then induces a map of (nonequivariant) orthogonal spectra

$$
F_{n}: T^{C_{m n}} \longrightarrow T^{C_{m}}
$$

This system of maps is compatible in the sense that

$$
F_{p} \circ F_{n}=F_{n p}: T^{C_{m n p}} \longrightarrow T^{C_{m}}
$$

for all $m, n, p$. Now let $T$ be a cyclotomic spectrum. Restricting to the inner product spaces $V$ with trivial $G$-action, the maps $r_{n, V}$ assemble to a map of (nonequivariant) orthogonal spectra $T^{C_{n}} \rightarrow T$; more generally, looking at the maps

$$
r_{n, V}{ }^{C_{m}}: T(V)^{C_{m n}}=\left(\rho_{n}^{*} T(V)^{C_{n}}\right)^{C_{m}} \longrightarrow T(V)^{C_{m}},
$$

we get maps of (nonequivariant) orthogonal spectra

$$
R_{n}: T^{C_{m n}} \longrightarrow T^{C_{m}}
$$

This system of maps is compatible in the sense that

$$
R_{p} \circ R_{n}=R_{n p}: T^{C_{m n p}} \longrightarrow T^{C_{m}}
$$

for all $m, n, p$. Moreover, by construction, the maps $R_{n}$ and $F_{p}$ commute:

$$
F_{n} \circ R_{p}=R_{p} \circ F_{n}: T^{C_{m n p}} \longrightarrow T^{C_{m}} .
$$

For $p$ a fixed prime $F_{p}$ and $R_{p}$ provide the maps $F$ and $R$ for constructing $T R$ and $T C$. To get the correct homotopy type, we need the fixed point orthogonal spectra to have the correct homotopy type, and we can only expect this to happen when $T$ satisfies additional constraints, such as being an equivariant $\Omega$-spectrum. To arrange this, we use an $\Omega$-spectrum replacement functor. 
Definition 4.5 An $\Omega$-spectrum replacement functor in the category of cyclotomic spectra consists of a functor $Q$ from cyclotomic spectra to itself and a natural transformation of cyclotomic spectra $\theta: \operatorname{Id} \rightarrow Q$ such that for any cyclotomic spectrum $T$, the map $\theta: T \rightarrow Q T$ is a weak equivalence of orthogonal $G$-spectra and $Q T$ is a fibrant orthogonal $G$-spectrum, ie, for any orthogonal $G$-representations $V, W$, the structure map $Q T(V) \rightarrow \Omega^{W} Q T(V \oplus W)$ is a weak equivalence of $G$-spaces.

As indicated in the previous section, we then construct $T R$ and $T C$ for a cyclotomic spectrum $T$ using the maps $F$ and $R$ for the cyclotomic spectrum $Q T$. For this to work, we need to know that an $\Omega$-spectrum replacement functor in the category of cyclotomic spectra exists. We describe two, $Q^{\mathcal{N}}$ and $Q^{\mathcal{I}}$, both of which are lifts of fibrant replacement functors on the category of orthogonal $G$-spectra. Recall that $\mathcal{I}$ is the category whose objects are the finite sets $\mathbf{m}=\{1, \ldots, m\}$ (including $\mathbf{0}=\{\}$ ) and whose maps are the inclusions. Let $\mathcal{N}$ be the subset of standard inclusions, so that there is a unique map $\mathbf{m} \rightarrow \mathbf{n}$ whenever $m \leq n$. Let $\mathcal{I}^{\omega}$ be the subcategory of $\mathcal{I}^{\infty}=\prod_{i=0}^{\infty} \mathcal{I}$ of sequences $\overrightarrow{\mathbf{m}}=\left(\mathbf{m}_{0}, \mathbf{m}_{1}, \ldots\right)$ such that all but finitely many of the $\mathbf{m}_{i}$ are $\mathbf{0}$, and let $\mathcal{N}^{\omega}$ be the corresponding subcategory of $\mathcal{N}^{\infty}$. For $\overrightarrow{\mathbf{m}}$ in $\mathcal{I}^{\omega}$, let

$$
\mathbb{C}(\overrightarrow{\mathbf{m}})=\mathbb{C}(0)^{m_{0}} \oplus \mathbb{C}(1)^{m_{1}} \oplus \mathbb{C}(2)^{m_{2}} \oplus \cdots<\mathcal{U} .
$$

Then $Q^{\mathcal{N}} T$ and $Q^{\mathcal{I}} T$ are the orthogonal $G$-spectra with $V$-th spaces

$$
\begin{aligned}
Q^{\mathcal{N}} T(V) & =\operatorname{hocolim}_{\overrightarrow{\mathbf{m}} \in \mathcal{N}} \Omega^{\mathbb{C}(\overrightarrow{\mathbf{m}})}(T(V \oplus \mathbb{C}(\overrightarrow{\mathbf{m}}))), \\
Q^{\mathcal{I}} T(V) & =\operatorname{hocolim}_{\overrightarrow{\mathbf{m}} \in \mathcal{I}} \Omega^{\mathbb{C}(\overrightarrow{\mathbf{m}})}(T(V \oplus \mathbb{C}(\overrightarrow{\mathbf{m}})))
\end{aligned}
$$

with structure maps induced by the $V$ variable. The inclusion of the object $\overrightarrow{\mathbf{0}}=$ $(\mathbf{0}, \mathbf{0}, \ldots)$ in the homotopy colimit then induces a map $T(V) \rightarrow Q^{\mathcal{N}} T(V) \rightarrow Q^{\mathcal{I}} T(V)$ that induces the natural transformations $T \rightarrow Q^{\mathcal{N}} T$ and $T \rightarrow Q^{\mathcal{I}} T$. We then have the following well-known fact.

Proposition 4.6 The functors $Q^{\mathcal{N}}$ and $Q^{\mathcal{I}}$ are fibrant replacement functors in orthogonal $G$-spectra.

Proof This is clear for $Q^{\mathcal{N}}$ by calculating homotopy groups. The space-level fixed point functors commute with the homotopy colimits and the obvious generalization of the argument of [39, 2.2.9] to $\mathcal{I}^{\omega}$ shows that the map $\left(Q^{\mathcal{N}} T(V)\right)^{C_{n}} \rightarrow\left(Q^{\mathcal{I}} T(V)\right)^{C_{n}}$ is a weak equivalence for all $V$ and $n$.

Let $\gamma_{n}: \mathcal{I}^{\omega} \rightarrow \mathcal{I}^{\omega}$ be the functor that sends $\overrightarrow{\mathbf{m}} \in \mathcal{I}$ to

$$
\gamma_{n}(\overrightarrow{\mathbf{m}})=\left(\mathbf{m}_{0}, \mathbf{m}_{n}, \mathbf{m}_{2 n}, \ldots\right) .
$$


Then $\mathbb{C}\left(\gamma_{n}(\overrightarrow{\mathbf{m}})\right)=\rho_{n}^{*}\left(\mathbb{C}(\overrightarrow{\mathbf{m}})^{C_{n}}\right)$, and we get maps $r_{n, V}$ for $Q^{\mathcal{N}} T$ and $Q^{\mathcal{I}} T$ as the map induced by the functor $\gamma_{n}$ and the natural transformation

$$
\begin{aligned}
\rho_{n}^{*}\left(\left(\Omega^{\mathbb{C}(\overrightarrow{\mathbf{m}})}(T(V \oplus \mathbb{C}(\overrightarrow{\mathbf{m}})))\right)^{C_{n}}\right) \longrightarrow \rho_{n}^{*}\left(\Omega^{\mathbb{C}(\overrightarrow{\mathbf{m}})^{C_{n}}}\left(T(V \oplus \mathbb{C}(\overrightarrow{\mathbf{m}}))^{C_{n}}\right)\right) \\
=\Omega^{\mathbb{C}\left(\gamma_{n}(\overrightarrow{\mathbf{m}})\right)} \rho_{n}^{*}\left(T(V \oplus \mathbb{C}(\overrightarrow{\mathbf{m}}))^{C_{n}}\right) \stackrel{r_{n}}{\longrightarrow} \Omega^{\mathbb{C}\left(\gamma_{n}(\overrightarrow{\mathbf{m}})\right)} T\left(\rho_{n}^{*} V^{C_{n}} \oplus \mathbb{C}\left(\gamma_{n}(\overrightarrow{\mathbf{m}})\right)\right) .
\end{aligned}
$$

A check of the diagrams then proves the following theorem.

Theorem 4.7 The natural transformations above make $Q^{\mathcal{N}}$ and $Q^{\mathcal{I}}$ into $\Omega$-spectrum replacement functors in the category of cyclotomic spectra.

We also make the (trivial) observation for use in Section 10 that as endofunctors on orthogonal $G$-spectra, both $Q^{\mathcal{N}}$ and $Q^{\mathcal{I}}$ are enriched in based spaces (ie, they are continuous and preserve the base point on mapping spaces).

The advantage of $Q^{\mathcal{N}}$ is that it is very closely related to the Lewis-May spectrification functor for "good" prespectra used in [20] to construct the TR and TC of a cyclotomic spectrum; the proof of the following proposition is easy, but is omitted as it would require a long review of [20, Section 2.2].

Proposition 4.8 For any cyclotomic spectrum $T$, the underlying Lewis-May prespectra of $T R$ and $T C$ constructed using $Q^{\mathcal{N}} T$ above is weakly equivalent through a natural zigzag to the underlying prespectrum of $T R$ and $T C$, respectively, constructed from the underlying equivariant Lewis-May prespectrum of $T$ using the construction of [20].

The advantage of $Q^{\mathcal{I}}$ is that viewed as a fibrant replacement functor on orthogonal $G$-spectra, it is lax monoidal. A famous observation of Lewis [29] (in modern terms) is that no fibrant replacement functor in orthogonal $G$-spectra can be symmetric monoidal. There are symmetric monoidal fibrant replacement functors for the positive stable model structure by work of Kro [27] (and this would suffice to get homotopically correct fixed point functors), but we do not know how to construct one that provides a positive $\Omega$-spectrum replacement functor in the category of cyclotomic spectra.

Finally, we need to check that for a small spectral category $\mathcal{C}, T H H(\mathcal{C})$ is naturally a cyclotomic spectrum. The remainder of the section is devoted to the proof of the following theorem.

Theorem 4.9 THH(-), with the natural structure maps $r_{n, V}$ described below, is a functor from the category of small spectral categories to the category of cyclotomic spectra. 
Proof Recall from the previous section that $T H H(\mathcal{C})$ is defined as the geometric realization of the cyclic object

$$
\operatorname{THH}_{q}(\mathcal{C})(V)=\operatorname{hocolim}_{\overrightarrow{\mathbf{n}} \in \mathcal{I}^{q+1}} \mathcal{G}\left(\mathcal{C} ; S^{V}\right)_{\overrightarrow{\mathbf{n}}},
$$

where

$\mathcal{G}\left(\mathcal{C} ; S^{V}\right)_{\overrightarrow{\mathbf{n}}}=\Omega^{n_{0}+\cdots+n_{q}}\left(\bigvee\left|\mathcal{C}\left(c_{q-1}, c_{q}\right)_{n_{q}} \wedge \cdots \wedge \mathcal{C}\left(c_{0}, c_{1}\right)_{n_{1}} \wedge \mathcal{C}\left(c_{q}, c_{0}\right)_{n_{0}}\right| \wedge S^{V}\right)$

Here $V$ is meant to be an orthogonal $G$-representation, and the $G$-action on $T H H(C)(V)$ is the diagonal action of the $G$-action on the cyclic structure and the $G$-action on $S^{V}$. Restricting to the subgroup $C_{n}$, the action then has a concrete description in terms of the $n$-th edgewise subdivision $\operatorname{sd}_{n} \operatorname{THH}(\mathcal{C})(V)$. This is the geometric realization of the simplicial $C_{n}$-space

$$
\operatorname{sd}_{n} T H H_{\bullet}(\mathcal{C})(V)=T H H_{n(\bullet+1)-1}(\mathcal{C})(V)=\operatorname{hocolim}_{\overrightarrow{\mathbf{p}} \in \mathcal{I}^{n(\bullet+1)}} \mathcal{G}\left(\mathcal{C} ; S^{V}\right)_{\overrightarrow{\mathbf{p}}},
$$

where the $C_{n}$-action is induced both by rotating the coordinates of $\mathcal{I}^{n(\bullet+1)}$ (and the corresponding loops and factors of $\mathcal{C}$ inside $\mathcal{G}\left(\mathcal{C} ; S^{V}\right)$ ) and by the action on $S^{V}$. An element of the homotopy colimit can only be a $C_{n}$-fixed point when it comes from a $\overrightarrow{\mathbf{p}}$ of the form

$$
n \overrightarrow{\mathbf{m}}=\left(\mathbf{m}_{0}, \ldots, \mathbf{m}_{q}, \mathbf{m}_{0}, \ldots, \mathbf{m}_{q}, \ldots, \mathbf{m}_{0}, \ldots, \mathbf{m}_{q}\right)
$$

for a sequence $\overrightarrow{\mathbf{m}}=\left(\mathbf{m}_{0}, \ldots, \mathbf{m}_{q}\right)$ repeated $n$ times. For such a $\overrightarrow{\mathbf{p}}$,

$$
\begin{aligned}
\mathcal{G}\left(\mathcal{C} ; S^{V}\right)_{\overrightarrow{\mathbf{p}}}=\Omega^{n\left(m_{0}+\cdots+m_{q}\right)}(\bigvee \mid & \mathcal{C}\left(c_{n(q+1)-2}, c_{n(q+1)-1}\right)_{m_{q}} \\
& \wedge \wedge \mathcal{C}\left(c_{(n(q+1)-q-1}, c_{n(q+1)-q}\right)_{m_{0}} \\
& \wedge \mathcal{C}\left(c_{(n-1)(q+1)-2}, c_{(n-1)(q+1)-1}\right)_{m_{q}} \\
& \left.\wedge \cdots \wedge \mathcal{C}\left(c_{0}, c_{1}\right)_{m_{1}} \wedge \mathcal{C}\left(c_{n(q+1)-1}, c_{0}\right)_{m_{0}} \mid \wedge S^{V}\right)
\end{aligned}
$$

and the $C_{n}$-action on the homotopy colimit restricts to a $C_{n}$-action on $\mathcal{C}\left(\mathcal{C} ; S^{V}\right)_{n \overrightarrow{\mathbf{m}}}$. Viewing $\Omega^{n m}$ as based maps out of $S^{n m}$, the $C_{n}$ fixed points are the $C_{n}$-equivariant maps out of $S^{n m}$; for such a map, restricting to fixed points gives a based map from $S^{m}=\left(S^{n m}\right)^{C_{n}}$ to

$$
\begin{aligned}
\left(\bigvee \mid \mathcal{C}\left(c_{n(q+1)-2}, c_{n(q+1)-1}\right)_{m_{q}}\right. & \wedge \\
& \left.\cdots \wedge \mathcal{C}\left(c_{n(q+1)-1}, c_{0}\right)_{m_{0}} \mid \wedge S^{V}\right)^{C_{n}} \\
& =\bigvee\left|\mathcal{C}\left(c_{q-1}, c_{q}\right)_{m_{q}} \wedge \cdots \wedge \mathcal{C}\left(c_{0}, c_{q}\right)_{m_{0}}\right| \wedge S^{V^{C_{n}}}
\end{aligned}
$$

Thus, restricting to fixed points induces a map

$$
\mathcal{G}\left(\mathcal{C} ; S^{V}\right)_{n \overrightarrow{\mathbf{m}}}^{C_{n}} \longrightarrow \mathcal{G}\left(\mathcal{C} ; S^{V^{C n}}\right)_{\overrightarrow{\mathbf{m}}}
$$


We define the map $r_{n, V}$ to be the induced simplicial map

(4.10) $\left(\operatorname{hocolim}_{\mathbf{p} \in \mathcal{I}^{n(\bullet+1)}} \mathcal{G}\left(\mathcal{C} ; S^{V}\right)_{\mathbf{p}}\right)^{C_{n}} \cong \operatorname{hocolim}_{\mathbf{m} \in \mathcal{I}^{\bullet+1}} \mathcal{G}\left(\mathcal{C} ; S^{V}\right)_{n \mathbf{m}}^{C_{n}}$ $\longrightarrow \operatorname{hocolim}_{\mathbf{m} \in \mathcal{I} \bullet+1} \mathcal{G}\left(\mathcal{C} ; S^{V^{C n}}\right)_{\mathbf{m}}$.

Following through the resulting $G$-action, we get as required a map

$$
r_{n, V}: \rho_{n}^{*}\left(\operatorname{THH}(\mathcal{C}, V)^{C_{n}}\right) \longrightarrow \operatorname{THH}\left(\mathcal{C}, \rho_{n}^{*}\left(V^{C_{n}}\right)\right),
$$

natural in maps of the small spectral category $\mathcal{C}$. Conditions (i), (ii), and (iii) of the definition of cyclotomic spectra are straightforward checks of the diagrams. It remains to check condition (iv).

For any $W$ in $\mathcal{U}^{C_{n}}$, let $\bar{W}$ be the union of the $G$-subspaces $V$ of $\mathcal{U}$ with $V^{C_{n}}=W$. Then $\bar{W}$ is an infinite dimensional subspace of $\mathcal{U}$ but we can generalize the notation $\operatorname{THH}(\mathcal{C})(V)$ to obtain the $G$-space $\operatorname{THH}(\mathcal{C})(\bar{W})$ and the map $r_{n, V}$ generalizes to a map

$$
r_{n, \bar{W}}: \rho_{n}^{*}\left(T H H(\mathcal{C}, \bar{W})^{C_{n}}\right) \longrightarrow T H H\left(\mathcal{C}, \rho_{n}^{*}\left(\bar{W}^{C_{n}}\right)\right)=T H H\left(\mathcal{C}, \rho_{n}^{*} W\right) .
$$

We then have

$$
\operatorname{THH}(\mathcal{C})(\bar{W})=\underset{V<\mathcal{U}, V^{C_{n}=W}}{\operatorname{colim}} \operatorname{THH}(\mathcal{C})(V),
$$

and we get a canonical isomorphism

$$
\underset{V<\mathcal{U}}{\operatorname{colim}} \pi_{q} \Omega^{V^{C_{n}}}\left(\operatorname{THH}(\mathcal{C})(V)^{C_{n}}\right) \cong \underset{W<\mathcal{U}^{C_{n}}}{\operatorname{colim}} \pi_{q} \Omega^{W}\left((\operatorname{THH}(\mathcal{C})(\bar{W}))^{C_{n}}\right)
$$

for $q \geq 0$ and likewise for the colimit for negative $q$. Thus, it suffices to show that the map $r_{n, \bar{W}}$ is a nonequivariant weak equivalence.

The space $S^{\bar{W}}$ is a model for the space $\Sigma^{W} \tilde{E} \mathcal{F}\left[C_{n}\right]$, meaning that for $H$ a closed subgroup of $G$, the fixed point space $\left(S^{\bar{W}}\right)^{H}$ is $\left(S^{W}\right)^{H}$ if $H$ contains $C_{n}$ and is contractible otherwise. It follows that for any based $\mathrm{CW} C_{n}$-spaces $Y, Z$, the map

$$
F\left(Y, Z \wedge S^{\bar{W}}\right)^{C_{n}} \longrightarrow F\left(Y^{C_{n}}, Z^{C_{n}} \wedge S^{W}\right)
$$

is a weak-equivalence, where $F$ denotes the $C_{n}$-space of based maps (cf [30, II.9.3]). In particular, since the map $r_{n, \bar{W}}$ is up to isomorphism the geometric realization of a homotopy colimit of maps of this form (4.10), it is a weak equivalence, and this completes the argument. 


\section{Spectral categories, homotopy categories, and invariance of $\mathrm{THH}$}

In this section, we continue the discussion of the basic properties of spectral categories and study the natural conditions under which spectral functors induce equivalences on THH. We review the concept of "Dwyer-Kan equivalence" (Definition 5.1) of spectral categories, which provides a more sophisticated notion of weak equivalence of spectral categories; Theorem 5.9 below indicates that Dwyer-Kan equivalences induce equivalences of $T H H$. The mapping spectra of a spectral category $\mathcal{C}$ give rise to an associated "homotopy category" that is an invariant of the Dwyer-Kan equivalences. Under rather general conditions (q.v. Definition 5.4), the homotopy category has a triangulated structure and this allows us to formulate useful "cofinality" and "thick subcategory" criteria for spectral functors to induce equivalences of $T H H$ in Theorems 5.11 and 5.12. Proofs of Theorems 5.9, 5.11 and 5.12 require the technical tools developed in the next section and are given there.

Definition 5.1 Let $F: \mathcal{C} \rightarrow \mathcal{D}$ be a spectral functor. We say that $F$ is a DwyerKan embedding or $D K$-embedding when for every $a, b \in \mathrm{ob} \mathcal{C}$, the map $\mathcal{C}(a, b) \rightarrow$ $\mathcal{D}(F a, F b)$ is a weak equivalence.

We say that $F$ is a Dwyer-Kan equivalence or DK-equivalence when $F$ is a DKembedding and for every $d \in \mathrm{ob} \mathcal{D}$, there exists a $c \in \mathrm{ob} \mathcal{C}$ such that $\mathcal{D}(-, d)$ and $\mathcal{D}(-, F c)$ represent naturally isomorphic enriched functors from $\mathcal{D}^{\text {op }}$ to the stable category.

We can rephrase this definition in terms of "homotopy categories": Associated to a spectral category $\mathcal{C}$, we have the following notion of homotopy category.

Definition 5.2 For a spectral category $\mathcal{C}$, the homotopy category $\pi_{0} \mathcal{C}$ is the Abcategory with the same objects, with morphism abelian groups $\pi_{0} \mathcal{C}(a, b)$, and with units and composition induced by the unit and composition maps of $\mathcal{C}$. The graded homotopy category is the $\mathrm{Ab}_{*}$-category with objects ob $\mathcal{C}$ and morphisms $\pi_{*} \mathcal{C}(a, b)$.

We remind the reader that by convention, $\pi_{0} \mathcal{C}(a, b)$ and $\pi_{*} \mathcal{C}(a, b)$ denote the homotopy groups of $\mathcal{C}(a, b)$ viewed as an object of the stable category.

Without any further hypotheses on the spectral categories in question, the following proposition is a straightforward consequence of the definitions and the Yoneda lemma for enriched functors [26, 2.4]. 
Proposition 5.3 A spectral functor $\mathcal{C} \rightarrow \mathcal{D}$ is a Dwyer-Kan equivalence if and only if it induces an equivalence of graded homotopy categories $\pi_{*} \mathcal{C} \rightarrow \pi_{*} \mathcal{D}$.

As we will see in Theorems 5.5 and 5.6 below, the homotopy category in practice often has a triangulated structure compatible with the mapping spectra. We formalize this in the following definition.

Definition 5.4 A spectral category $\mathcal{C}$ is pretriangulated means the following:

(i) There is an object 0 in $\mathcal{C}$ such that the right $\mathcal{C}$-module $\mathcal{C}(-, 0)$ is homotopically trivial (weakly equivalent to the constant functor with value the one-point symmetric $\operatorname{spectrum} *)$.

(ii) Whenever a right $\mathcal{C}$-module $\mathcal{M}$ has the property that $\Sigma \mathcal{M}$ is weakly equivalent to a representable $\mathcal{C}$-module $\mathcal{C}(-, c)$ (for some object $c$ in $\mathcal{C}$ ), then $\mathcal{M}$ is weakly equivalent to a representable $\mathcal{C}$-module $\mathcal{C}(-, d)$ for some object $d$ in $\mathcal{C}$.

(iii) Whenever the right $\mathcal{C}$-modules $\mathcal{M}$ and $\mathcal{N}$ are weakly equivalent to representable $\mathcal{C}$-modules $\mathcal{C}(-, a)$ and $\mathcal{C}(-, b)$ respectively, then the homotopy cofiber of any map of right $\mathcal{C}$-modules $\mathcal{M} \rightarrow \mathcal{N}$ is weakly equivalent to a representable $\mathcal{C}$-module.

The first condition guarantees the existence of a zero object in the homotopy category $\pi_{0} \mathcal{C}$; the usual argument shows that the left module $\mathcal{C}(0,-)$ is also homotopically trivial. The second condition gives a desuspension functor on $\pi_{0} \mathcal{C}$ and the third condition in particular produces a suspension functor on $\pi_{0} \mathcal{C}$ : We choose $\Sigma^{-1} a$ and $\Sigma a$ representing $\Sigma^{-1} \mathcal{C}(-, a)$ and $\Sigma \mathcal{C}(-, a)$, respectively, in the derived category of right $\mathcal{C}$-modules. Then $\Sigma^{-1} a$ and $\Sigma a$ in particular represent the functors $\pi_{1} \mathcal{C}(-, a)$ and $\pi_{-1} \mathcal{C}(-, a)$, respectively, from $\pi_{0} \mathcal{C}$ to sets, and so are unique up to unique isomorphism in $\pi_{0} \mathcal{C}$.

In the third condition, note that maps of right modules from $\mathcal{C}(-, a)$ to $\mathcal{C}(-, b)$ are in one to one correspondence with the vertices of the zeroth simplicial set of $\mathcal{C}(a, b)$; using weakly equivalent $\mathcal{M}$ and $\mathcal{N}$, the maps then represent arbitrary elements of $\pi_{0} \mathcal{C}(a, b)$. In the case when all of the mapping spectra of $\mathcal{C}$ are fibrant symmetric spectra (eg, after replacing $\mathcal{C}$ by $\mathcal{C}^{\Omega}$ ), condition (iii) can be simplified to considering just the homotopy cofibers of maps $\mathcal{C}(-, a) \rightarrow \mathcal{C}(-, b)$. We explain this interpretation of condition (iii) in more detail at the end of the section in the proof of the following theorem.

Theorem 5.5 Any small spectral category $\mathcal{C}$ DK-embeds in a small pretriangulated spectral category $\widetilde{\mathcal{C}}$. 
The category $\tilde{\mathcal{C}}$ is closely related to the category of right $\mathcal{C}$-modules, essentially the closure of (the Yoneda embedding of) $\mathcal{C}$ under desuspensions and cofibration sequences. The third condition in the definition of pretriangulated spectral category then indicates the sequences in $\mathcal{C}$ that are equivalent to cofibration sequences in $\widetilde{\mathcal{C}}$. We can therefore use the third condition to define the analogue of Puppe (cofibration) sequences: We say that a sequence

$$
a \longrightarrow b \longrightarrow c \longrightarrow \Sigma a
$$

in $\pi_{0} \mathcal{C}$ is a four term Puppe sequence if there exists right $\mathcal{C}$-modules $\mathcal{M}$ and $\mathcal{N}$ and a map of right $\mathcal{C}$-modules $f: \mathcal{M} \rightarrow \mathcal{N}$ such that the four term Puppe sequence of $f$

$$
\mathcal{M} \longrightarrow \mathcal{N} \longrightarrow C f \longrightarrow \Sigma \mathcal{M}
$$

in the category of right $\mathcal{C}$-modules is isomorphic in the derived category of right $\mathcal{C}$-modules to the sequence

$$
\mathcal{C}(-, a) \longrightarrow \mathcal{C}(-, b) \longrightarrow \mathcal{C}(-, c) \longrightarrow \mathcal{C}(-, \Sigma a)
$$

such that the isomorphism $\Sigma \mathcal{M} \rightarrow \mathcal{C}(-, \Sigma a) \cong \Sigma \mathcal{C}(-, a)$ is the suspension of the isomorphism $\mathcal{M} \rightarrow \mathcal{C}(-, a)$. We prove the following theorem at the end of the section.

Theorem 5.6 If the spectral category $\mathcal{C}$ is pretriangulated, then its homotopy category is triangulated with distinguished triangles the four term Puppe sequences. A spectral functor between pretriangulated spectral categories induces a triangulated functor on homotopy categories.

Corollary 5.7 A spectral functor $\mathcal{C} \rightarrow \mathcal{D}$ between pretriangulated spectral categories is a Dwyer-Kan equivalence if and only if it induces an equivalence of homotopy categories $\pi_{0} \mathcal{C} \rightarrow \pi_{0} \mathcal{D}$.

In the context of DG-categories, various analogous conditions have been given to ensure that the homotopy category of the DG-category is triangulated; see Keller [25], Bondal [5] and Drinfeld [10]. Following [5; 10], we refer to such DG-categories as pretriangulated. We have the following consistency result. It follows from the fact that the various functors relating chain complexes to $H \mathbb{Z}$-modules to symmetric spectra all preserve homotopy cofibration sequences.

Proposition 5.8 If $\mathcal{D}$ is a pretriangulated $D G$-category, then its associated spectral category is pretriangulated.

As indicated by Proposition 5.3 and Corollary 5.7, we take the perspective that the mapping spectra encode the homotopy theory of the spectral category. From this 
viewpoint, DK-equivalences clearly represent the correct general notion of weak equivalence of spectral categories. An alternative perspective would not require the mapping spectra of a spectral category $\mathcal{C}$ to encode all of the homotopy theory, but rather also include an additional notion of weak equivalence of objects of $\mathcal{C}$. For example, this is appropriate in the context of enriched model categories. For model categories enriched over symmetric spectra, the homotopy theory is a localization of the intrinsic homotopy theory of the associated spectral category. The full spectral subcategory of the cofibrant-fibrant objects is the spectral category whose mapping spectra encode the homotopy theory of the enriched model category. This subcategory tends not to be preserved under most interesting functors. Under properness hypotheses, a "cofiber" version of $T H H$ works somewhat better; see Section 11 for more details.

We prove the following invariance theorem for DK-equivalences in the next section.

Theorem 5.9 A DK-equivalence $\mathcal{C} \rightarrow \mathcal{D}$ induces a weak equivalence $\mathrm{THH}(\mathcal{C}) \rightarrow$ $\operatorname{THH}(\mathcal{D})$.

We also prove the following more general theorem for bimodule coefficients. In the statement, the $(\mathcal{C}, \mathcal{C})$-bimodule $F^{*} \mathcal{N}$ is the bimodule obtained by restriction of scalars; it is the spectral functor from $\mathcal{C}^{\text {op }} \wedge \mathcal{C}$ to symmetric spectra defined by first applying $F$ to each variable and then applying $\mathcal{N}$.

Theorem 5.10 Let $F: \mathcal{C} \rightarrow \mathcal{D}$ be a $D K$-equivalence of small spectral categories, $\mathcal{M}$ a $(\mathcal{C}, \mathcal{C})$-bimodule and $\mathcal{N}$ a $(\mathcal{D}, \mathcal{D})$-bimodule. A weak equivalence $\mathcal{M} \rightarrow F^{*} \mathcal{N}$ induces a weak equivalence $\operatorname{THH}(\mathcal{C} ; \mathcal{M}) \rightarrow \operatorname{THH}(\mathcal{D} ; \mathcal{N})$.

We now move on from weak equivalences to Morita equivalences. For objects $a$ and $c$ of $\mathcal{D}$, say that $c$ is a homotopy factor of $a$ if it is a factor in the graded homotopy category $\pi_{*} \mathcal{D}$, ie, if there exists an object $b$ in $\mathcal{D}$ and a natural isomorphism $\pi_{*} \mathcal{D}(-, c) \cong \pi_{*} \mathcal{D}(-, a) \times \pi_{*} \mathcal{D}(-, b)$ of contravariant functors from $\pi_{*} \mathcal{D}$ to the category of graded abelian groups. We say that a spectral functor $F: \mathcal{C} \rightarrow \mathcal{D}$ is homotopy cofinal if it induces weak equivalences on mapping spaces and each object of $\mathcal{D}$ is a homotopy factor of the image of some object in $\mathcal{C}$. We prove the following theorem in the next section.

Theorem 5.11 A homotopy cofinal spectral functor $\mathcal{C} \rightarrow \mathcal{D}$ of small spectral categories induces a weak equivalence $T H H(\mathcal{C}) \rightarrow T H H(\mathcal{D})$.

The previous theorem admits a more sophisticated variant. Given a set $C$ of objects in a pretriangulated spectral category $\mathcal{D}$, the thick closure of $C$ is the set of objects in the 
thick subcategory of $\pi_{0} \mathcal{D}$ generated by $C$. In terms of the spectral category $\mathcal{D}$, the thick closure of $C$ is the smallest set $\bar{C}$ of objects of $\mathcal{D}$ containing $C$ and satisfying the following:

(i) If $a$ is a homotopy factor of an object of $\bar{C}$, then $a$ is in $\bar{C}$.

(ii) If the right $\mathcal{D}$-module $\Sigma \mathcal{D}(-, a)$ is weakly equivalent to $\mathcal{D}(-, c)$ for some $c$ in $\bar{C}$, then $a$ is in $\bar{C}$.

(iii) If the right $\mathcal{D}$-module $\mathcal{D}(-, a)$ is weakly equivalent to the cofiber of a map of right $\mathcal{D}$-modules $\mathcal{M} \rightarrow \mathcal{M}^{\prime}$ with $\mathcal{M}, \mathcal{M}^{\prime}$ weakly equivalent to $\mathcal{D}(-, c)$, $\mathcal{D}\left(-, c^{\prime}\right)$ for $c, c^{\prime}$ in $\bar{C}$, then $a$ is in $\bar{C}$.

A set is thick if it is its own thick closure. Since any small spectral category $\mathcal{C}$ embeds as a full spectral subcategory of a small pretriangulated spectral category $\mathcal{D}$, the following theorem, proved in the next section, in particular allows us to always reduce questions in $\mathrm{THH}$ to the case of small pretriangulated spectral categories.

Theorem 5.12 Let $\mathcal{D}$ be a pretriangulated spectral category. Let $C$ be a set of objects of $\mathcal{D}, \bar{C}$ its thick closure, and $C^{\prime}$ a set containing $C$ and contained in $\bar{C}$. Let $\mathcal{C}$ and $\mathcal{C}^{\prime}$ be the full spectral subcategories of $\mathcal{D}$ on the objects in $C$ and $C^{\prime}$ respectively. Then the inclusion $\mathcal{C} \rightarrow \mathcal{C}^{\prime}$ induces a weak equivalence $T H H(\mathcal{C}) \rightarrow T H H\left(\mathcal{C}^{\prime}\right)$.

We close the section with the proofs of Theorems 5.5 and 5.6. The argument involves the well-known properties of categories of enriched functors into a Quillen closed model category. For any small spectral category $\mathcal{C}$, the category $\mathfrak{M o d}_{\mathcal{C}}$ of right $\mathcal{C}$-modules has a standard model structure (or projective model structure) that is proper and compactly generated, where the generating cofibrations and generating acyclic cofibrations are the maps $\mathcal{C}(-, c) \wedge f$ for $c$ in $\mathcal{C}$ and $f$ varying through the generating cofibrations and generating acyclic cofibrations (respectively) of the stable model structure on symmetric spectra described in [24, 3.3.2,3.4.9] (see also [24, 3.4.2.1,3.4.16]). Consequently, the weak equivalences and fibrations are the maps that are objectwise weak equivalences and objectwise fibrations (respectively) in the stable model structure on symmetric spectra. The representable right $\mathcal{C}$-modules $\mathcal{C}(-, c)$ are cofibrant and compact, meaning that maps out of $\mathcal{C}(-, c)$ preserve sequential colimits. In fact, the set of maps, simplicial set of maps, and symmetric spectrum of maps out of $\mathcal{C}(-, c)$ preserve arbitrary colimits, by the enriched Yoneda lemma [26, 2.4].

Proof of Theorem 5.6 In the case when $\mathcal{C}$ is small, we use the model theory above as follows. Using Quillen's theory of cofibration sequences, we obtain a triangulated structure on the Quillen homotopy category Ho $\mathfrak{M o d}_{\mathcal{C}}$ of $\mathfrak{M o d}_{\mathcal{C}}$. The homotopy category $\pi_{0} \mathcal{C}$ embeds as a full subcategory of $\mathrm{Ho} \mathfrak{M o d}_{\mathcal{C}}$, and the conditions in the definition 
of pretriangulated spectral category imply that $\pi_{0} \mathcal{C}$ is closed under desuspensions, suspensions, and triangles in $\mathrm{Ho}_{\mathfrak{C}} \mathfrak{M o d}_{\mathcal{C}}$. In the case when $\mathcal{C}$ is not small, $\mathfrak{M o d}_{\mathcal{C}}$ does not typically have small Hom-sets. To handle this, one restricts to the cell modules and uses small object arguments to construct replacements whose values on a given small set of objects are fibrant symmetric spectra.

Now given a spectral functor $F: \mathcal{C} \rightarrow \mathcal{D}$ between small pretriangulated spectral categories, left Kan extension produces a functor $\operatorname{Lan}_{F}: \mathfrak{M o d}_{\mathcal{C}} \rightarrow \mathfrak{M o d}_{\mathcal{D}}$ left adjoint to the functor $F^{*}: \mathfrak{M o d}_{\mathcal{D}} \rightarrow \mathfrak{M o d}_{\mathcal{C}}$. Since $F^{*}$ preserves fibrations and weak equivalences in the model structure above, $\operatorname{Lan}_{F}$ and $F^{*}$ form a Quillen adjoint pair. In particular $\mathrm{Lan}_{F}$ preserves Quillen cofibration sequences and Quillen suspensions. It follows that the left derived functor of $\operatorname{Lan}_{F}$ on Quillen homotopy categories is triangulated; on the representable functors, the left derived functor $\operatorname{Lan}_{F}$ is just $\pi_{0} F: \pi_{0} \mathcal{C} \rightarrow \pi_{0} \mathcal{D}$. Again, the case when $\mathcal{C}$ or $\mathcal{D}$ is not small may be handled by a straightforward direct argument in terms of the cell modules.

Proof of Theorem 5.5 We again use the model theory on categories of modules. By Proposition 2.6, we can assume without loss of generality that all of the mapping spectra $\mathcal{C}(x, y)$ are fibrant in the stable model structure on symmetric spectra, and so the representable right $\mathcal{C}$-modules $\mathcal{C}(-, c)$ are both cofibrant and fibrant in the model structure on $\mathfrak{M o d}_{\mathcal{C}}$. In order to remain in the setting of small categories, we restrict to a small full subcategory of $\mathfrak{M o d}_{\mathcal{C}}$ as follows. For any set $U$, write $U \mathfrak{M o d}_{\mathcal{C}}$ for the full subcategory of $\mathfrak{M o d}_{\mathcal{C}}$ consisting of the functors that take values in symmetric spectra whose underlying sets are in $U$. Then $U \mathfrak{M o d}_{\mathcal{C}}$ is a small spectral category, and if we choose $U$ to be the power set of a sufficiently large cardinal, then $U \mathfrak{M o d}_{\mathcal{C}}$ will be closed (up to universal isomorphisms) under the usual (cardinal limited) constructions of homotopy theory in $\mathfrak{M o d}_{\mathcal{C}}$, including the small objects argument constructing factorizations. In particular, $U \mathfrak{M o d}_{\mathcal{C}}$ is a Quillen model category (assuming just finite limits and colimits) with cofibrations, fibrations, and weak equivalences the maps that are such in $\mathfrak{M o d}_{\mathcal{C}}$. We then get a (closed model) category $\mathfrak{M o d}_{U \mathfrak{M o d}_{\mathcal{C}}}$.

Let $\tilde{\mathcal{C}}$ be the full spectral subcategory of $U \mathfrak{M o d}_{\mathcal{C}}$ consisting of the cofibrant-fibrant objects. The enriched Yoneda lemma embeds $\mathcal{C}$ as a full spectral subcategory of $\tilde{\mathcal{C}}$. Properties (i) and (ii) for $\widetilde{\mathcal{C}}$ in the definition of pretriangulated spectral category are clear. For property (iii), consider a map of right $\widetilde{\mathcal{C}}$-modules $\mathcal{M} \rightarrow \mathcal{N}$. Since the model structure on $\mathfrak{M}^{\circ} \mathfrak{d}_{\tilde{\mathcal{C}}}$ is left proper, after replacing $\mathcal{M}$ and $\mathcal{N}$ with fibrant replacements, we obtain an equivalent homotopy cofiber, and so we can assume without loss of generality that $\mathcal{M}$ and $\mathcal{N}$ are fibrant. We assume that $\mathcal{M}$ is weakly equivalent to $\widetilde{\mathcal{C}}(-, a)$ and $\mathcal{N}$ is weakly equivalent to $\widetilde{\mathcal{C}}(-, b)$ for objects $a, b$ in $\widetilde{\mathcal{C}}$; since $\widetilde{\mathcal{C}}(-, a)$ and $\widetilde{\mathcal{C}}(-, b)$ are cofibrant and $\mathcal{M}$ and $\mathcal{N}$ are fibrant, we can choose weak equivalences 
$\tilde{\mathcal{C}}(-, a) \rightarrow \mathcal{M}$ and $\tilde{\mathcal{C}}(-, b) \rightarrow \mathcal{N}$. Furthermore, as $\widetilde{\mathcal{C}}(a, b)$ and $\mathcal{N}(a)$ are both fibrant, we can lift the composite map $\widetilde{\mathcal{C}}(-, a) \rightarrow \mathcal{N}$ to a homotopic map $\widetilde{\mathcal{C}}(-, a) \rightarrow \widetilde{\mathcal{C}}(-, b)$. We get a weak equivalence on the homotopy cofibers. The map $\widetilde{\mathcal{C}}(-, a) \rightarrow \widetilde{\mathcal{C}}(-, b)$ comes from a map $a \rightarrow b$ by the Yoneda lemma. A fibrant replacement of the homotopy cofiber in $U \mathfrak{M o d}_{\mathcal{C}}$ is in $\widetilde{\mathcal{C}}$ and represents the homotopy cofiber of $\mathcal{M} \rightarrow \mathcal{N}$ in $\mathfrak{M o d}_{\tilde{\mathcal{C}}}$. This completes the proof of Theorem 5.5.

\section{The Dennis-Waldhausen Morita argument}

In this section, we consider the invariance properties of $T H H$ from the perspective of generalized Morita theory. Dennis and Waldhausen gave a very concrete argument for the Morita invariance of the Hochschild homology of rings using an explicit bisimplicial construction [45, page 391]. We give a broad generalization of this argument to the setting of spectral categories that provides the technical foundations for the proofs of the theorems of the previous section as well as the arguments in the remainder of the paper.

The argument uses the two-sided bar construction.

Definition 6.1 Let $\mathcal{C}$ be a small spectral category, $\mathcal{M}$ a right $\mathcal{C}$-module, and $\mathcal{N}$ a left $\mathcal{C}$-module. The two-sided bar construction $B(\mathcal{M} ; \mathcal{C} ; \mathcal{N})$ is the diagonal (geometric realization) of the simplicial symmetric spectrum $B_{\bullet}(\mathcal{M} ; \mathcal{C} ; \mathcal{N})$, where

$$
B_{q}(\mathcal{M} ; \mathcal{C} ; \mathcal{N})=\bigvee \mathcal{M}\left(c_{q}\right) \wedge \mathcal{C}\left(c_{q-1}, c_{q}\right) \wedge \cdots \wedge \mathcal{C}\left(c_{0}, c_{1}\right) \wedge \mathcal{N}\left(c_{0}\right),
$$

where the sum is over the $(q+1)$-tuples $\left(c_{0}, \ldots, c_{q}\right)$ of objects of $\mathcal{C}$. We make this a simplicial object with the usual two-sided bar construction face and degeneracy maps: the zeroth face map is induced by the action of $\mathcal{C}$ on $\mathcal{N}$, the last face map is induced by the action of $\mathcal{C}$ on $\mathcal{M}$, and the remaining face maps are induced by the composition in $\mathcal{C}$. The degeneracy maps are induced by the unit maps $S \rightarrow \mathcal{C}\left(c_{i}, c_{i}\right)$.

The following is the main technical proposition of this section. In it and elsewhere when necessary for clarity, we write

$$
B(\mathcal{M}(x) ; x, y \in \mathcal{C} ; \mathcal{N}(y)) \quad \text { and } \quad N^{\mathrm{cy}}(x, y \in \mathcal{C} ; \mathcal{P}(x, y))
$$

for $B(\mathcal{M} ; \mathcal{C} ; \mathcal{N})$ and $N^{\text {cy }}(\mathcal{C} ; \mathcal{P})$, especially when $\mathcal{M}, \mathcal{N}$, and/or $\mathcal{P}$ depend on other variables. 
Proposition 6.2 (Dennis-Waldhausen Morita Argument) Let $\mathcal{C}$ and $\mathcal{D}$ be small spectral categories. Let $\mathcal{P}$ be a $(\mathcal{D}, \mathcal{C})$-bimodule and $\mathcal{Q}$ a $(\mathcal{C}, \mathcal{D})$-bimodule. Then there is a natural isomorphism of symmetric spectra

$$
N^{\mathrm{cy}}(\mathcal{C}, B(\mathcal{P}, \mathcal{D}, \mathcal{Q})) \cong N^{\mathrm{cy}}(\mathcal{D} ; B(\mathcal{Q}, \mathcal{C}, \mathcal{P}))
$$

that is,

$$
\begin{aligned}
& N^{\mathrm{cy}}(x, y \in \mathcal{C} ; B(\mathcal{P}(w, y) ; w, z \in \mathcal{D} ; \mathcal{Q}(x, z))) \\
& \cong N^{\mathrm{cy}}(w, z \in \mathcal{D} ; B(\mathcal{Q}(x, z) ; x, y \in \mathcal{C} ; \mathcal{P}(w, y))) .
\end{aligned}
$$

Proof We can identify both symmetric spectra

$$
N^{\mathrm{cy}}(\mathcal{C} ; B(\mathcal{P} ; \mathcal{D} ; \mathcal{Q})) \quad \text { and } \quad N^{\mathrm{cy}}(\mathcal{D} ; B(\mathcal{Q} ; \mathcal{C} ; \mathcal{P}))
$$

as the diagonal of the bisimplicial spectrum with $(q, r)$-simplices as pictured.

$$
\begin{aligned}
& \mathcal{C}\left(c_{q-1}, x\right) \wedge \cdots \wedge \mathcal{C}\left(y, c_{1}\right) \\
& \wedge \wedge \\
& \mathcal{Q}(x, z) \quad \mathcal{P}(w, y) \\
& \wedge \wedge \\
& \mathcal{D}\left(z, d_{1}\right) \wedge \cdots \wedge \mathcal{D}\left(d_{r-1}, w\right)
\end{aligned}
$$

These two constructions are therefore canonically isomorphic in the point-set category of symmetric spectra.

The following lemma complements Proposition 6.2. Its proof is the usual simplicial contraction (see for example May [33, 9.8]).

Lemma 6.3 (Two-Sided Bar Lemma) Let $\mathcal{C}$ be a small spectral category, let $\mathcal{M}$ be a right $\mathcal{C}$-module, and let $\mathcal{N}$ be a left $\mathcal{C}$-module. For any object $c$ in $\mathcal{C}$, the composition maps

$$
B \bullet(\mathcal{M} ; \mathcal{C} ; \mathcal{C}(c,-)) \longrightarrow \mathcal{M}(c) \quad \text { and } \quad B \bullet(\mathcal{C}(-, c) ; \mathcal{C} ; \mathcal{N}) \longrightarrow \mathcal{N}(c)
$$

are simplicial homotopy equivalences.

We use the previous proposition and lemma to prove the following theorem, which gives a criterion for converting objectwise equivalence conditions into equivalences on $\mathrm{THH}$. 
Theorem 6.4 Let $\mathcal{C}$ and $\mathcal{D}$ be small spectral categories and let $F: \mathcal{C} \rightarrow \mathcal{D}$ be a spectral functor. Let $\mathcal{M}$ be a $(\mathcal{C}, \mathcal{C})$-bimodule, $\mathcal{N}$ a $(\mathcal{D}, \mathcal{D})$-bimodule and $\mathcal{M} \rightarrow F^{*} \mathcal{N}$ a weak equivalence. Assume that $\mathcal{C}$ and $\mathcal{D}$ are pointwise cofibrant. Suppose that the map of symmetric spectra

$$
B(\mathcal{D}(F-, z) ; \mathcal{C} ; \mathcal{N}(w, F-)) \longrightarrow B(\mathcal{D}(-, z) ; \mathcal{D} ; \mathcal{N}(w,-))
$$

is a weak equivalence for each fixed $w, z$ in $\mathcal{D}$. Then the map

$$
\operatorname{THH}(\mathcal{C} ; \mathcal{M}) \longrightarrow \operatorname{THH}(\mathcal{D} ; \mathcal{N})
$$

is a weak equivalence.

Proof Consider the commutative diagram

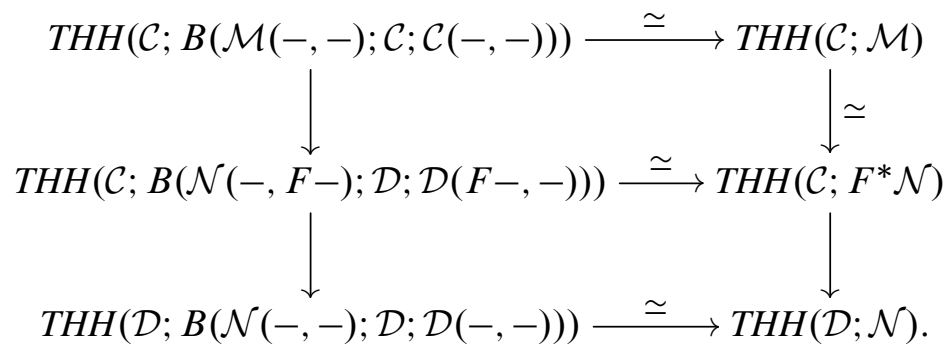

The arrows marked " $\simeq$ " are weak equivalences by the Two-Sided Bar Lemma and Proposition 3.7 above. The composite map on the right is the map we are interested in, and so our goal is to prove that the two maps on the left are weak equivalences. The first map is induced by a map of $\mathcal{C}$-bimodules

$$
B(\mathcal{M}(-,-) ; \mathcal{C} ; \mathcal{C}(-,-)) \longrightarrow B(\mathcal{N}(-, F-) ; \mathcal{D} ; \mathcal{D}(F-,-)),
$$

which is easily seen to be a weak equivalence by the Two-Sided Bar Lemma and the hypothesis that $\mathcal{M} \rightarrow F^{*} \mathcal{N}$ is a weak equivalence. Since we have assumed that $\mathcal{C}$ and $\mathcal{D}$ are pointwise cofibrant, by Proposition 3.5, to show that the second map is a weak equivalence, it suffices to show that the map

$$
N^{\mathrm{cy}}(\mathcal{C} ; B(\mathcal{N}(-, F-) ; \mathcal{D} ; \mathcal{D}(F-,-))) \longrightarrow N^{\mathrm{cy}}(\mathcal{D} ; B(\mathcal{N}(-,-) ; \mathcal{D} ; \mathcal{D}(-,-)))
$$

is a weak equivalence and here we apply Proposition 6.2: We obtain a commutative diagram with the horizontal maps isomorphisms

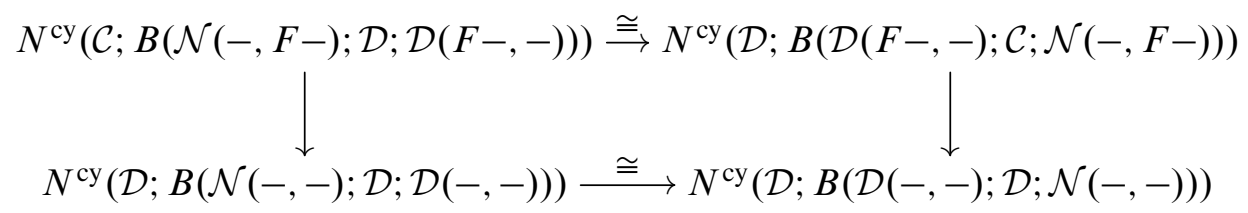


by applying Proposition 6.2 with $\mathcal{P}=\mathcal{N}(-, F-)$ and $\mathcal{Q}=\mathcal{D}(F-,-)$ on the top and $\mathcal{P}=\mathcal{N}(-,-)$ and $\mathcal{Q}=\mathcal{D}(-,-)$ (for $\mathcal{C}=\mathcal{D}$ ) on the bottom. By the hypothesis on the map $(*)$, the map of $(\mathcal{D}, \mathcal{D})$-bimodules

$$
B(\mathcal{D}(F-,-) ; \mathcal{C} ; \mathcal{N}(-, F-)) \longrightarrow B(\mathcal{D}(-,-) ; \mathcal{D} ; \mathcal{N}(-,-))
$$

is a weak equivalence, and it follows that the vertical maps above are weak equivalences.

We now apply this criterion in the proof of Theorem 5.9, Theorem 5.10, Theorem 5.11 and Theorem 5.12.

Proof of Theorem 5.9 Using Proposition 2.7, we can assume without loss of generality that $\mathcal{C}$ and $\mathcal{D}$ are pointwise cofibrant, and then apply Theorem 6.4 with $\mathcal{M}=\mathcal{C}$ and $\mathcal{N}=\mathcal{D}$. We need to show that the map

$$
B(\mathcal{D}(F-, z) ; \mathcal{C} ; \mathcal{D}(w, F-)) \longrightarrow B(\mathcal{D}(-, z) ; \mathcal{D} ; \mathcal{D}(w,-))
$$

is a weak equivalence for each $w, z$ in $\mathcal{D}$, and using the Two-Sided Bar Lemma, it suffices to show that the composite map

$$
B(\mathcal{D}(F-, z) ; \mathcal{C} ; \mathcal{D}(w, F-)) \longrightarrow \mathcal{D}(w, z)
$$

is a weak equivalence. Viewing $B_{\bullet}(\mathcal{D}(F-, z) ; \mathcal{C} ; \mathcal{D}(w, F-))$ as a simplicial object in the stable category, up to simplicial isomorphism, it only depends on $\mathcal{D}(F-, z)$ as a functor from $\mathcal{C}^{\text {op }}$ to the stable category. By hypothesis, there exists an object $c$ in $\mathcal{C}$ such that $\mathcal{D}(-, z)$ and $\mathcal{D}(-, F c)$ are isomorphic as functors from $\mathcal{D}^{\text {op }}$ to the stable category. It follows that $\mathcal{D}(F-, z)$ and $\mathcal{D}(F-, F c) \simeq \mathcal{C}(-, c)$ are isomorphic as functors from $\mathcal{C}^{\text {op }}$ to the stable category. Since this is just a comparison of simplicial objects in the stable category, we do not get a direct comparison on geometric realizations (but see also the proof of Theorem 5.10 below). Nonetheless, the homotopy groups of $B$. are the $E_{1}$-term of a spectral sequence that computes the homotopy groups of $B$. The $E_{1}$ differential comes from the simplicial face maps, and applying the Two-Sided Bar Lemma, we see that this spectral sequence degenerates at $E_{2}$ and that (6.6) is a weak equivalence.

For the proof of Theorem 5.10, we note that the map (*) in the statement of Theorem 6.4 still makes sense when we replace $\mathcal{D}(-, z)$ with an arbitrary right $\mathcal{D}$-module $\phi$ :

$$
B\left(F^{*} \phi ; \mathcal{C} ; \mathcal{N}(w, F-)\right) \longrightarrow B(\phi ; \mathcal{D} ; \mathcal{N}(w,-))
$$

We have used $\phi$ to denote the right $\mathcal{D}$-module to avoid possible confusion between the different roles played by the right module $\phi$ and the bimodule $\mathcal{N}$. If $\mathcal{N}(w,-)$ 
is objectwise cofibrant, then $B\left(F^{*} \phi ; \mathcal{C} ; \mathcal{N}(w, F-)\right)$ and $B(\phi ; \mathcal{D} ; \mathcal{N}(w,-))$ preserve weak equivalences in $\phi$.

Proof of Theorem 5.10 We can assume without loss of generality that $\mathcal{C}$ and $\mathcal{D}$ are pointwise cofibrant and that $\mathcal{M}$ and $\mathcal{N}$ are objectwise cofibrant. Applying Theorem 6.4, it suffices to show that the map $(*)$

$$
B(\mathcal{D}(F-, z) ; \mathcal{C} ; \mathcal{N}(w, F-)) \longrightarrow B(\mathcal{D}(-, z) ; \mathcal{D} ; \mathcal{N}(w,-))
$$

is a weak equivalence for each $w, z$ in $\mathcal{D}$. Fixing $w, z$, it suffices to show that the map (6.7) is a weak equivalence for $\phi=\mathcal{D}(-, z)$. Let $\psi$ be a right $\mathcal{D}$-module fibrant replacement of $\mathcal{D}(-, z)$. By hypothesis, viewing $\psi$ as an enriched functor from $\mathcal{D}$ to the stable category, we have a natural isomorphism $\tilde{f}: \mathcal{D}\left(-, F z^{\prime}\right) \rightarrow \psi$ for some $z^{\prime}$ in $\mathcal{C}$; by the Yoneda lemma for enriched functors, this corresponds to an element $\tilde{f} \in \pi_{0}\left(\psi\left(F z^{\prime}\right)\right)$. Since $\psi\left(F z^{\prime}\right)$ is fibrant, we can choose a vertex $f$ in $\psi\left(F z^{\prime}\right)_{0}$ representing $\tilde{f}$. Again by the Yoneda lemma, $f$ represents a map of right $\mathcal{D}$-modules $\mathcal{D}\left(-, F z^{\prime}\right) \rightarrow \psi$ that induces the natural isomorphism $\tilde{f}$ of enriched functors to the stable category. In particular, $f$ is a weak equivalence. The map (6.7) is a weak equivalence for $\phi=\mathcal{D}\left(-, F z^{\prime}\right)$, and so is a weak equivalence for $\phi=\psi$ and for $\phi=\mathcal{D}(-, z)$.

Theorem 5.11 can be proved using essentially the same argument as the proof of Theorem 5.9 above, using the fact that a direct sum of maps in the stable category is an isomorphism if and only if it is an isomorphism on each factor. On the other hand, given Theorem 5.5, Theorem 5.11 follows from Theorem 5.9 and Theorem 5.12, which we now prove.

Proof of Theorem 5.12 Without loss of generality, we can assume that $\mathcal{D}$ is pointwise cofibrant, and then $\mathcal{C}$ and $\mathcal{C}^{\prime}$ are also pointwise cofibrant. Applying Theorem 6.4, it suffices to show that the map $(*)$

$$
B\left(\mathcal{C}^{\prime}(-, z) ; \mathcal{C} ; \mathcal{C}^{\prime}(w,-)\right) \longrightarrow B\left(\mathcal{C}^{\prime}(-, z) ; \mathcal{C}^{\prime} ; \mathcal{C}^{\prime}(w,-)\right)
$$

is a weak equivalence for every $w, z$ in $\mathcal{C}^{\prime}$, or more generally, that the map (6.7)

$$
B\left(\phi ; \mathcal{C} ; \mathcal{C}^{\prime}(w,-)\right) \longrightarrow B\left(\phi ; \mathcal{C}^{\prime} ; \mathcal{C}^{\prime}(w,-)\right)
$$

is a weak equivalence for any right $\mathcal{C}^{\prime}$-module $\phi$ that is weakly equivalent to $\mathcal{C}^{\prime}(-, z)$ for $z$ in $\mathcal{C}^{\prime}$. By the Two-Sided Bar Lemma, we know that (6.8) is a weak equivalence when $\phi$ is $\mathcal{C}(-, x)$ for $x$ in $\mathcal{C}$. Using the fact that both sides preserve homotopy cofibration sequences in the $\phi$ variable, it follows that (6.8) is a weak equivalence for $\mathcal{D}(-, z)$ for any $z$ in the thick subcategory of $\pi_{0} \mathcal{D}$ generated by $\pi_{0} \mathcal{C}$. This completes the proof of Theorem 5.12. 


\section{The general localization theorem}

In this section, we discuss a general theorem that produces localization cofibration sequences in $T H H$. The basic strategy takes advantage of the fact that $T H H$ preserves (co)fibration sequences in the bimodule variable: We apply the Dennis-Waldhausen Morita argument to identify $\mathrm{THH}$ of a small spectral category with $\mathrm{THH}$ of another small spectral category with coefficients in a bimodule; see Lemma 7.6 below. Roughly, we then obtain our localization cofibration sequences by reinterpreting sequences of $\mathrm{THH}$ of small spectral categories

$$
\operatorname{THH}(\mathcal{A}) \longrightarrow T H H(\mathcal{B}) \longrightarrow T H H(\mathcal{C})
$$

up to weak equivalence as the $T H H$ of a single small spectral category with coefficients in a sequence of judiciously chosen bimodules

$$
\operatorname{THH}\left(\mathcal{B} ; \mathcal{L}_{\mathcal{A}}^{\mathcal{B}}\right) \longrightarrow \operatorname{THH}(\mathcal{B} ; \mathcal{B}) \longrightarrow \operatorname{THH}\left(\mathcal{B} ; \mathcal{Q}_{\mathcal{A}}^{\mathcal{B}}\right)
$$

where $\mathcal{Q}_{\mathcal{A}}^{\mathcal{B}}$ is the cofiber of a map of $(\mathcal{B}, \mathcal{B})$-bimodules $\mathcal{L}_{\mathcal{A}}^{\mathcal{B}} \rightarrow \mathcal{B}$. Although we can make more general statements, the situation we are most interested in is when the sequence of small spectral categories models a triangulated quotient (in the sense of Verdier). We prove the following theorem.

Theorem 7.1 Let $F: \mathcal{B} \rightarrow \mathcal{C}$ be a spectral functor between small pretriangulated spectral categories, and let $\mathcal{A}$ be the full spectral subcategory of $\mathcal{B}$ consisting of the objects a such that $F(a)$ is isomorphic to zero in the homotopy category $\pi_{0} \mathcal{C}$. If the induced map from the triangulated quotient $\pi_{0} \mathcal{B} / \pi_{0} \mathcal{A}$ to $\pi_{0} \mathcal{C}$ is cofinal, then $T H H(\mathcal{C})$ is weakly equivalent the homotopy cofiber of $\operatorname{THH}(\mathcal{A}) \rightarrow T H H(\mathcal{B})$. The analogous relationship holds also for $T R(\mathcal{C})$ and $T C(\mathcal{C})$.

In general, we call $(\mathcal{B}, \mathcal{A})$ a localization pair when $\mathcal{B}$ is a pretriangulated spectral category and $\mathcal{A}$ is a full spectral subcategory such that $\pi_{0} \mathcal{A}$ is thick in $\pi_{0} \mathcal{B}$; we say that the localization pair is small when the spectral category $\mathcal{B}$ is small. This definition of localization pair differs slightly from that of Keller $[25,2.4]$ in that we do not require a well-behaved ambient category (our additional requirement that $\mathcal{A}$ be thick is for convenience rather than necessity by Theorem 5.12).

In Theorem 7.1, letting $\mathcal{Z}$ be the full subcategory of objects of $\mathcal{C}$ in the thick closure of the image of $\mathcal{A}$, then $(\mathcal{C}, \mathcal{Z})$ is a localization pair and $(\mathcal{B}, \mathcal{A}) \rightarrow(\mathcal{C}, \mathcal{Z})$ is a map of localization pairs: It is a spectral functor $\mathcal{B} \rightarrow \mathcal{C}$ that takes $\mathcal{A}$ into $\mathcal{Z}$. Note that for any objects $x, y$ in $\mathcal{Z}$, the spectrum $\mathcal{Z}(x, y)$ is homotopically trivial, and so $T H H(\mathcal{Z})$ is homotopically trivial. The inclusion of $T H H(\mathcal{C})$ in the homotopy cofiber of 
$T H H(\mathcal{Z}) \rightarrow T H H(\mathcal{C})$ is therefore a weak equivalence. We have the map of homotopy cofibers

$$
C(\operatorname{THH}(\mathcal{A}) \rightarrow \operatorname{THH}(\mathcal{B})) \longrightarrow C(\operatorname{THH}(\mathcal{Z}) \rightarrow T H H(\mathcal{C})),
$$

and we prove Theorem 7.1 by showing that this map is a weak equivalence: We get the statement for $T R(\mathcal{C})$ and $T C(\mathcal{C})$ because the weak equivalence on $T H H$ above implies that the map of cyclotomic spectra

$$
C(\operatorname{THH}(\mathcal{A}) \rightarrow T H H(\mathcal{B})) \longrightarrow C(T H H(\mathcal{Z}) \rightarrow T H H(\mathcal{C}))
$$

is a weak equivalence.

Theorem 7.1 then naturally appears as a special case of the following theorem, which essentially says that the cofiber of $T H H$ is an invariant of the localization pair.

Theorem 7.2 Let $F:\left(\mathcal{B}_{1}, \mathcal{A}_{1}\right) \rightarrow\left(\mathcal{B}_{2}, \mathcal{A}_{2}\right)$ be a map of small localization pairs. If the induced map of triangulated quotients

$$
\pi_{0} \mathcal{B}_{1} / \pi_{0} \mathcal{A}_{1} \longrightarrow \pi_{0} \mathcal{B}_{2} / \pi_{0} \mathcal{A}_{2}
$$

is cofinal, then the induced map of cyclotomic spectra

$$
C\left(\operatorname{THH}\left(\mathcal{A}_{1}\right) \rightarrow \operatorname{THH}\left(\mathcal{B}_{1}\right)\right) \longrightarrow C\left(\operatorname{THH}\left(\mathcal{A}_{2}\right) \rightarrow \operatorname{THH}\left(\mathcal{B}_{2}\right)\right)
$$

is a weak equivalence.

For ease of reference, we also state the following immediate corollary.

Corollary 7.3 Under the hypotheses and notation of the previous theorem, the maps of spectra

$$
\begin{aligned}
& C\left(\operatorname{TR}\left(\mathcal{A}_{1}\right) \rightarrow \operatorname{TR}\left(\mathcal{B}_{1}\right)\right) \longrightarrow C\left(\operatorname{TR}\left(\mathcal{A}_{2}\right) \rightarrow \operatorname{TR}\left(\mathcal{B}_{2}\right)\right) \\
& C\left(\operatorname{TC}\left(\mathcal{A}_{1}\right) \rightarrow \operatorname{TC}\left(\mathcal{B}_{1}\right)\right) \longrightarrow C\left(\operatorname{TC}\left(\mathcal{A}_{2}\right) \rightarrow \operatorname{TC}\left(\mathcal{B}_{2}\right)\right)
\end{aligned}
$$

are weak equivalences.

Following Keller [25], we define $T H H$ of a localization pair as the cofiber on $T H H$. The previous theorem provides a perspective and justification for the following definition.

Definition 7.4 Let $(\mathcal{B}, \mathcal{A})$ be a small localization pair. We write $\operatorname{CTHH}(\mathcal{B} / \mathcal{A})$ for the cyclotomic spectrum obtained as the cofiber of the map $\operatorname{THH}(\mathcal{A}) \rightarrow \operatorname{THH}(\mathcal{B})$. 
We now move on to the proof of Theorem 7.2. Applying Proposition 2.7, we assume without loss of generality that $\mathcal{B}$ and $\mathcal{C}$ are pointwise cofibrant and it follows that $\mathcal{A}$ is pointwise cofibrant. We write $C N^{\text {cy }}(\mathcal{B} / \mathcal{A})$ for the cofiber of the map $N^{\text {cy }}(\mathcal{A}) \rightarrow$ $N^{\text {cy }}(\mathcal{B})$. Proposition 3.5 now reduces the proof of Theorem 7.2 to the following lemma, whose proof occupies the remainder of the section.

Lemma 7.5 Let $F:\left(\mathcal{B}_{1}, \mathcal{A}_{1}\right) \rightarrow\left(\mathcal{B}_{2}, \mathcal{A}_{2}\right)$ be a map of small localization pairs with $\mathcal{B}_{1}$ and $\mathcal{B}_{2}$ pointwise cofibrant. If the induced map of triangulated quotients

$$
\pi_{0} \mathcal{B}_{1} / \pi_{0} \mathcal{A}_{1} \longrightarrow \pi_{0} \mathcal{B}_{2} / \pi_{0} \mathcal{A}_{2}
$$

is cofinal, then the induced map $C N^{\mathrm{cy}}\left(\mathcal{B}_{1} / \mathcal{A}_{1}\right) \rightarrow C N^{\mathrm{cy}}\left(\mathcal{B}_{2} / \mathcal{A}_{2}\right)$ is a weak equivalence.

As a first step, we use the Dennis-Waldhausen Morita argument, Proposition 6.2, to rewrite $N^{\text {cy }}(\mathcal{A})$ as $N^{\text {cy }}\left(\mathcal{B} ; \mathcal{L}_{\mathcal{A}}^{\mathcal{B}}\right)$ for an appropriate $(\mathcal{B}, \mathcal{B})$-bimodule $\mathcal{L}_{\mathcal{A}}^{\mathcal{B}}$.

Lemma 7.6 Let $(\mathcal{B}, \mathcal{A})$ be a small localization pair with $\mathcal{B}$ pointwise cofibrant, and let $\mathcal{L}_{\mathcal{A}}^{\mathcal{B}}$ be the $(\mathcal{B}, \mathcal{B})$-bimodule defined by

$$
\mathcal{L}_{\mathcal{A}}^{\mathcal{B}}(x, y)=B(\mathcal{B}(-, y) ; \mathcal{A} ; \mathcal{B}(x,-)) .
$$

Then $N^{\mathrm{cy}}(\mathcal{A})$ is naturally (in maps of pointwise cofibrant localization pairs) weakly equivalent to $N^{\text {cy }}\left(\mathcal{B} ; \mathcal{L}_{\mathcal{A}}^{\mathcal{B}}\right)$.

Proof We apply Proposition 6.2 with $\mathcal{C}=\mathcal{A}, \mathcal{D}=\mathcal{B}, \mathcal{P}=\mathcal{B}$, and $\mathcal{Q}=\mathcal{B}$ to obtain a natural isomorphism

$$
N^{\mathrm{cy}}\left(\mathcal{B} ; \mathcal{L}_{\mathcal{A}}^{\mathcal{B}}\right)=N^{\mathrm{cy}}(\mathcal{B} ; B(\mathcal{B} ; \mathcal{A} ; \mathcal{B})) \cong N^{\mathrm{cy}}(\mathcal{A} ; B(\mathcal{B} ; \mathcal{B} ; \mathcal{B}))
$$

The natural map

$$
\operatorname{THH}(\mathcal{A} ; B(\mathcal{B} ; \mathcal{B} ; \mathcal{B})) \longrightarrow T H H(\mathcal{A} ; \mathcal{B})=T H H(\mathcal{A} ; \mathcal{A})=T H H(\mathcal{A}) .
$$

is a weak equivalence since the composition map of $(\mathcal{A}, \mathcal{A})$-bimodules $B(\mathcal{B} ; \mathcal{B} ; \mathcal{B}) \rightarrow \mathcal{B}$ is a weak equivalence by the Two-Sided Bar Lemma 6.3.

For a small localization pair $(\mathcal{B}, \mathcal{A})$, write $\mathcal{Q}_{\mathcal{A}}^{\mathcal{B}}$ for the $(\mathcal{B}, \mathcal{B})$-bimodule obtained as the cofiber of the composition map $\mathcal{L}_{\mathcal{A}}^{\mathcal{B}} \rightarrow \mathcal{B}$. Then by the previous lemma, we have a natural weak equivalence

$$
N^{\mathrm{cy}}\left(\mathcal{B} ; \mathcal{Q}_{\mathcal{A}}^{\mathcal{B}}\right) \simeq \operatorname{CTHH}(\mathcal{B} / \mathcal{A})
$$


Naturality here refers to the fact that a map of small localization pairs $F$ induces a map of $\left(\mathcal{B}_{1}, \mathcal{B}_{1}\right)$-bimodules $\mathcal{Q}_{\mathcal{A}_{1}}^{\mathcal{B}_{1}} \rightarrow F^{*} \mathcal{Q}_{\mathcal{A}_{2}}^{\mathcal{B}_{2}}$ and therefore a map

$$
N^{\mathrm{cy}}\left(\mathcal{B}_{1} ; \mathcal{Q}_{\mathcal{A}_{1}}^{\mathcal{B}_{1}}\right) \longrightarrow N^{\mathrm{cy}}\left(\mathcal{B}_{2} ; \mathcal{Q}_{\mathcal{A}_{2}}^{\mathcal{B}_{2}}\right) \text {. }
$$

Looking at the proof of Lemma 7.6, we see that this map is compatible under the weak equivalences above with the map on homotopy cofibers in the statement of Lemma 7.5. Thus, to prove Lemma 7.5, we just need to show that the map (7.7) is a weak equivalence.

For a small localization pair $(\mathcal{B}, \mathcal{A})$ and fixed object $b$ in $\mathcal{B}$, the right $\mathcal{B}$-module $\mathcal{L}_{\mathcal{A}}^{\mathcal{B}}(-, b)$ is the enriched homotopy left Kan extension along $\mathcal{A} \rightarrow \mathcal{B}$ of the enriched functor $\mathcal{B}(-, b)$ from $\mathcal{A}$ to symmetric spectra. (By which we mean the derived functor of the enriched left Kan extension [26, Section 4].) Philosophically, the homotopy cofiber of the map $\mathcal{L}_{\mathcal{A}}^{\mathcal{B}}(-, b) \rightarrow \mathcal{B}(-, b)$ should then represent the right $\mathcal{C}$-module of maps into the image of $b$ in any spectral category $\mathcal{C}$ representing the triangulated quotient; $\mathrm{cf}[10,(1.3)]$. From this perspective and viewed through the principles of the Dennis-Waldhausen Morita argument, $\operatorname{THH}\left(\mathcal{B} ; \mathcal{Q}_{\mathcal{A}}^{\mathcal{B}}\right)$ should be equivalent to $\operatorname{THH}(\mathcal{C})$. This is the idea behind the following lemma proved at the end of the section.

Lemma 7.8 Under the hypotheses of Lemma 7.5, the map of $\left(\mathcal{B}_{1}, \mathcal{B}_{1}\right)$-bimodules $\mathcal{Q}_{\mathcal{A}_{1}}^{\mathcal{B}_{1}} \rightarrow F^{*} \mathcal{Q}_{\mathcal{A}_{2}}^{\mathcal{B}_{2}}$ is a weak equivalence.

A fundamental property of $\mathcal{Q}_{\mathcal{A}}^{\mathcal{B}}$ is that $\mathcal{Q}_{\mathcal{A}}^{\mathcal{B}}(a,-)$ and $\mathcal{Q}_{\mathcal{A}}^{\mathcal{B}}(-, a)$ are homotopically trivial for any object $a$ in $\mathcal{A}$ : The Two-Sided Bar Lemma 6.3 implies that the composition maps $\mathcal{L}_{\mathcal{A}}^{\mathcal{B}}(a,-) \rightarrow \mathcal{B}(a,-)$ and $\mathcal{L}_{\mathcal{A}}^{\mathcal{B}}(-, a) \rightarrow \mathcal{B}(-, a)$ are weak equivalences. This leads to the following technical observation needed below to analyze the map (7.7).

Lemma 7.9 For a small localization pair $(\mathcal{B}, \mathcal{A})$, the maps of bimodules $B\left(\mathcal{B} ; \mathcal{B} ; \mathcal{Q}_{\mathcal{A}}^{\mathcal{B}}\right) \longrightarrow B\left(\mathcal{Q}_{\mathcal{A}}^{\mathcal{B}} ; \mathcal{B} ; \mathcal{Q}_{\mathcal{A}}^{\mathcal{B}}\right) \quad$ and $\quad B\left(\mathcal{Q}_{\mathcal{A}}^{\mathcal{B}} ; \mathcal{B} ; \mathcal{B}\right) \longrightarrow B\left(\mathcal{Q}_{\mathcal{A}}^{\mathcal{B}} ; \mathcal{B} ; \mathcal{Q}_{\mathcal{A}}^{\mathcal{B}}\right)$ induced by $\mathcal{B} \rightarrow \mathcal{Q}_{\mathcal{A}}^{\mathcal{B}}$ are weak equivalences.

Proof We prove the first equivalence; the argument for the second is similar. Expanding $\mathcal{Q}_{\mathcal{A}}^{\mathcal{B}}$ in terms of its definition, we see that $B\left(\mathcal{Q}_{\mathcal{A}}^{\mathcal{B}} ; \mathcal{B} ; \mathcal{Q}_{\mathcal{A}}^{\mathcal{B}}\right)$ is the cofiber of the bimodule map $B\left(\mathcal{L}_{\mathcal{A}}^{\mathcal{B}} ; \mathcal{B} ; \mathcal{Q}_{\mathcal{A}}^{\mathcal{B}}\right) \rightarrow B\left(\mathcal{B} ; \mathcal{B} ; \mathcal{Q}_{\mathcal{A}}^{\mathcal{B}}\right)$, and so by the Two-Sided Bar Lemma 6.3 , it suffices to see that $B\left(\mathcal{L}_{\mathcal{A}}^{\mathcal{B}} ; \mathcal{B} ; \mathcal{Q}_{\mathcal{A}}^{\mathcal{B}}\right)$ is homotopically trivial. Since $\mathcal{L}_{\mathcal{A}}^{\mathcal{B}}=B(\mathcal{B} ; \mathcal{A} ; \mathcal{B})$, expanding out $B\left(\mathcal{L}_{\mathcal{A}}^{\mathcal{B}} ; \mathcal{B} ; \mathcal{Q}_{\mathcal{A}}^{\mathcal{B}}\right)$ as a bisimplicial object, we get the isomorphism

$$
B\left(\mathcal{L}_{\mathcal{A}}^{\mathcal{B}} ; \mathcal{B} ; \mathcal{Q}_{\mathcal{A}}^{\mathcal{B}}\right)=B\left(B(\mathcal{B}, \mathcal{A}, \mathcal{B}) ; \mathcal{B} ; \mathcal{Q}_{\mathcal{A}}^{\mathcal{B}}\right) \cong B\left(\mathcal{B} ; \mathcal{A} ; B\left(\mathcal{B} ; \mathcal{B} ; \mathcal{Q}_{\mathcal{A}}^{\mathcal{B}}\right)\right)
$$


Applying the Two-Sided Bar Lemma 6.3 again, we see that $B\left(\mathcal{B} ; \mathcal{A} ; B\left(\mathcal{B} ; \mathcal{B} ; \mathcal{Q}_{\mathcal{A}}^{\mathcal{B}}\right)\right)$ is weakly equivalent to $B\left(\mathcal{B} ; \mathcal{A} ; \mathcal{Q}_{\mathcal{A}}^{\mathcal{B}}\right)$. This is homotopically trivial because the restriction of $\mathcal{Q}_{\mathcal{A}}^{\mathcal{B}}(x,-)$ to $\mathcal{A}$ is homotopically trivial for any $x$.

We can extend $\mathcal{Q}_{\mathcal{A}}^{\mathcal{B}}$ to be a $\left(\mathcal{B}, \mathfrak{M o d}_{\mathcal{B}}\right)$-bimodule, where $\mathfrak{M o d}_{\mathcal{B}}$ denotes the category of right $\mathcal{B}$-modules. For $x$ an object of $\mathcal{B}$ and $\phi$ a right $\mathcal{B}$-module, let $\mathcal{Q}_{\mathcal{A}}^{\mathcal{B}}(x, \phi)$ be the cofiber of the composition map

$$
B(\phi(-) ; \mathcal{A} ; \mathcal{B}(x,-)) \longrightarrow \phi(x) .
$$

Clearly, $\mathcal{Q}_{\mathcal{A}}^{\mathcal{B}}(x, \phi)$ is isomorphic to $\mathcal{Q}_{\mathcal{A}}^{\mathcal{B}}(x, y)$ when $\phi=\mathcal{B}(-, y)$, and $\mathcal{Q}_{\mathcal{A}}^{\mathcal{B}}(x,-)$ sends cofibration sequences of right $\mathcal{B}$-modules to cofibration sequences of symmetric spectra and sends weak equivalences of right $\mathcal{B}$-modules to weak equivalences of symmetric spectra. The usual category of fractions description of the triangulated quotient $\pi_{0} \mathcal{B} / \pi_{0} \mathcal{A}$ and the fact that $\mathcal{Q}_{\mathcal{A}}^{\mathcal{B}}$ is homotopically trivial when either variable is in $\mathcal{A}$ then implies that $\mathcal{Q}_{\mathcal{A}}^{\mathcal{B}}(-, y)$ and $\mathcal{Q}_{\mathcal{A}}^{\mathcal{B}}\left(-, y^{\prime}\right)$ are weakly equivalent right $\mathcal{B}-$ modules when $y$ and $y^{\prime}$ are isomorphic in $\pi_{0} \mathcal{B} / \pi_{0} \mathcal{A}$. Moreover, when $z$ is isomorphic to $w \vee y$ in $\pi_{0} \mathcal{B} / \pi_{0} \mathcal{A}, \mathcal{Q}_{\mathcal{A}}^{\mathcal{B}}(-, z)$ is weakly equivalent as a right $\mathcal{B}$-module to $\mathcal{Q}_{\mathcal{A}}^{\mathcal{B}}(-, w) \vee \mathcal{Q}_{\mathcal{A}}^{\mathcal{B}}(-, y)$. Using these observations and the lemmas above, we can now prove Lemma 7.5.

Proof of Lemma 7.5 We need to show that the map (7.7) is a weak equivalence. Consider the following commutative diagram

$$
\begin{aligned}
N^{\mathrm{cy}}\left(\mathcal{B}_{1} ; F^{*} B\left(\mathcal{Q}_{\mathcal{A}_{2}}^{\mathcal{B}_{2}} ; \mathcal{B}_{2} ; \mathcal{Q}_{\mathcal{A}_{2}}^{\mathcal{B}_{2}}\right)\right) \stackrel{\simeq}{\simeq} N^{\mathrm{cy}}\left(\mathcal{B}_{1} ; F^{*} B\left(\mathcal{B}_{2} ; \mathcal{B}_{2} ; \mathcal{Q}_{\mathcal{A}_{2}}^{\mathcal{B}_{2}}\right)\right) \stackrel{\simeq}{\simeq} N^{\mathrm{cy}}\left(\mathcal{B}_{1} ; F^{*} \mathcal{Q}_{\mathcal{A}_{2}}^{\mathcal{B}_{2}}\right) \\
N^{\mathrm{cy}}\left(\mathcal{B}_{2} ; B\left(\mathcal{Q}_{\mathcal{A}_{2}}^{\mathcal{B}_{2}} ; \mathcal{B}_{2} ; \mathcal{Q}_{\mathcal{A}_{2}}^{\mathcal{B}_{2}}\right)\right) \stackrel{\downarrow}{\longleftarrow} N^{\mathrm{cy}}\left(\mathcal{B}_{2} ; B\left(\mathcal{B}_{2} ; \mathcal{B}_{2} ; \mathcal{Q}_{\mathcal{A}_{2}}^{\mathcal{B}_{2}}\right)\right) \underset{\sim}{\simeq} N^{\mathrm{cy}}\left(\mathcal{B}_{2} ; \mathcal{Q}_{\mathcal{A}_{2}}^{\mathcal{B}_{2}}\right) .
\end{aligned}
$$

The lefthand horizontal maps are weak equivalences by Lemma 7.9 and the righthand horizontal maps are weak equivalences by the Two-Sided Bar Lemma 6.3. The map (7.7) is the composite of the righthand vertical map and the induced map on $N^{\text {cy }}$ of the map of bimodules $\mathcal{Q}_{\mathcal{A}_{1}}^{\mathcal{B}_{1}} \rightarrow F^{*} \mathcal{Q}_{\mathcal{A}_{2}}^{\mathcal{B}_{2}}$, which is a weak equivalence by Lemma 7.8. Thus, to see that (7.7) is a weak equivalence, it suffices to show that one of the vertical maps is a weak equivalence.

Focusing on the lefthand vertical map and applying the Dennis-Waldhausen Morita argument (Proposition 6.2), it suffices to show that the map

$$
B\left(\mathcal{Q}_{\mathcal{A}_{2}}^{\mathcal{B}_{2}}(F-, y) ; \mathcal{B}_{1} ; \mathcal{Q}_{\mathcal{A}_{2}}^{\mathcal{B}_{2}}(x, F-)\right) \longrightarrow B\left(\mathcal{Q}_{\mathcal{A}_{2}}^{\mathcal{B}_{2}}(-, y) ; \mathcal{B}_{2} ; \mathcal{Q}_{\mathcal{A}_{2}}^{\mathcal{B}_{2}}(x,-)\right)
$$

is a weak equivalence for every pair of objects $x, y$ in $\mathcal{B}_{2}$. It is clear from Lemmas 7.8 and 7.9 that (7.10) is an equivalence when either $x$ or $y$ is in the image of $\mathcal{B}_{1}$. By 
the remarks above, if an object $y$ in $\mathcal{B}_{2}$ is isomorphic in $\pi_{0} \mathcal{B}_{2} / \pi_{0} \mathcal{A}_{2}$ to $F y^{\prime}$ for some object $y^{\prime}$ in $\mathcal{B}_{1}$, then $\mathcal{Q}_{\mathcal{A}_{2}}^{\mathcal{B}_{2}}(-, y)$ is weakly equivalent as a right $\mathcal{B}_{2}$-module to $\mathcal{Q}_{\mathcal{A}_{2}}^{\mathcal{B}_{2}}\left(-, F y^{\prime}\right)$ and the map (7.10) is a weak equivalence for all $x$. Since $\pi_{0} \mathcal{B}_{1} / \pi_{0} \mathcal{A}_{1}$ is cofinal in $\pi_{0} \mathcal{B}_{2} / \pi_{0} \mathcal{A}_{2}$, for any $y$ in $\mathcal{B}_{2}$, there exists $w$ in $\mathcal{B}_{2}$ such that the sum $w \vee y$ in $\pi_{0} \mathcal{B}_{2} / \pi_{0} \mathcal{A}_{2}$ is isomorphic to $F z$ for some $z$ in $\mathcal{B}_{1}$; then as noted above, the right $\mathcal{B}_{2}$-module $\mathcal{Q}_{\mathcal{A}_{2}}^{\mathcal{B}_{2}}(-, w) \vee \mathcal{Q}_{\mathcal{A}_{2}}^{\mathcal{B}_{2}}(-, y)$ is weakly equivalent to $\mathcal{Q}_{\mathcal{A}_{2}}^{\mathcal{B}_{2}}(-, F z)$. We get compatible weak equivalences

$$
\begin{aligned}
& B\left(\mathcal{Q}_{\mathcal{A}_{2}}^{\mathcal{B}_{2}}(F-, w) ; \mathcal{B}_{1} ; \mathcal{Q}_{\mathcal{A}_{2}}^{\mathcal{B}_{2}}(x, F-)\right) \vee B\left(\mathcal{Q}_{\mathcal{A}_{2}}^{\mathcal{B}_{2}}(F-, y) ; \mathcal{B}_{1} ; \mathcal{Q}_{\mathcal{A}_{2}}^{\mathcal{B}_{2}}(x, F-)\right) \\
& \simeq \\
& B\left(\mathcal{Q}_{\mathcal{A}_{2}}^{\mathcal{B}_{2}}(F-, F z) ; \mathcal{B}_{1} ; \mathcal{Q}_{\mathcal{A}_{2}}^{\mathcal{B}_{2}}(x, F-)\right), \\
& B\left(\mathcal{Q}_{\mathcal{A}_{2}}^{\mathcal{B}_{2}}(-, w) ; \mathcal{B}_{2} ; \mathcal{Q}_{\mathcal{A}_{2}}^{\mathcal{B}_{2}}(x,-)\right) \vee B\left(\mathcal{Q}_{\mathcal{A}_{2}}^{\mathcal{B}_{2}}(-, y) ; \mathcal{B}_{2} ; \mathcal{Q}_{\mathcal{A}_{2}}^{\mathcal{B}_{2}}(x,-)\right) \\
& \simeq \\
& B\left(\mathcal{Q}_{\mathcal{A}_{2}}^{\mathcal{B}_{2}}(-, F z) ; \mathcal{B}_{2} ; \mathcal{Q}_{\mathcal{A}_{2}}^{\mathcal{B}_{2}}(x,-)\right),
\end{aligned}
$$

and we see that (7.10) is a weak equivalence for $x$ and $y$.

It still remains to prove Lemma 7.8. The proof makes use of Bousfield localization [6] (see also Hirschhorn [22, Section 3.3]) in the category of right $\mathcal{B}$-modules for a small pretriangulated spectral category $\mathcal{B}$. As discussed in Section 5 , the category $\mathfrak{M o d}_{\mathcal{B}}$ of right $\mathcal{B}$-modules has a standard compactly generated stable model structure where the weak equivalences and fibrations are the maps that are objectwise weak equivalences and fibrations in the stable model structure on symmetric spectra. The generating cofibrations and generating acyclic cofibrations are the maps $\mathcal{B}(-, b) \wedge f$ for $b$ in $\mathcal{B}$ and $f$ varying through the generating cofibrations and generating acyclic cofibrations, respectively, of the stable model structure on symmetric spectra. The representable right $\mathcal{B}$-modules $\mathcal{B}(-, b)$ are both cofibrant and compact.

Now let $(\mathcal{B}, \mathcal{A})$ be a localization pair. We say that a right $\mathcal{B}$-module $\psi$ is $\mathcal{A}$-local if it is fibrant and $\psi(a)$ is homotopically trivial for every object $a$ of $\mathcal{A}$. In this context, we say that a map of right $\mathcal{B}$-modules $f: \phi \rightarrow \phi^{\prime}$ is an $\mathcal{A}$-local equivalence if it induces a bijection of morphism sets in the Quillen homotopy category, $\left[\phi^{\prime}, \psi\right] \rightarrow[\phi, \psi]$, for every $\mathcal{A}$-local right $\mathcal{B}$-module $\psi$. The $\mathcal{A}$-local model structure on right $\mathcal{B}$-modules has the same cofibrations as the standard stable model structure but has weak equivalences the $\mathcal{A}$-local equivalences $[22,4.1 .2]$. This is a compactly generated model structure with the acyclic cofibrations generated by the acyclic cofibrations in the standard stable model structure together with the maps of the form $\mathcal{B}(-, a) \wedge f$ for $a$ in $\mathcal{A}$ and $f$ a generating cofibration in the stable model structure on symmetric spectra. The fibrant objects in the $\mathcal{A}$-local model structure are the $\mathcal{A}$-local right $\mathcal{B}$-modules. 
More specifically, every acyclic cofibration in the $\mathcal{A}$-local model structure is a retract of a sequential colimit of pushouts along arbitrary coproducts of the generating acyclic cofibrations indicated above. The cofiber of such a map in the colimit is weakly equivalent (in the standard stable model structure) to a wedge of objects of $\mathcal{A}$. Since modules represented by objects of $\mathcal{B}$ are compact, a standard argument [23, 2.3.17; $36,2.1$ ] shows that if a representable right $\mathcal{B}$-module $\mathcal{B}(-, b)$ is $\mathcal{A}$-acyclic ( $\mathcal{A}$-locally equivalent to $*$ ), then in the Quillen homotopy category of the standard stable model structure, $\mathcal{B}(-, b)$ is in the thick subcategory generated by the representables from $\mathcal{A}$, and so $b$ is in $\mathcal{A}$. This implies the following proposition.

Proposition 7.11 Let $(\mathcal{B}, \mathcal{A})$ be a localization pair. The spectral Yoneda functor that includes $\mathcal{B}$ in $\mathfrak{M o d}_{\mathcal{B}}$ as the representable functors induces a triangulated embedding of $\pi_{0} \mathcal{B} / \pi_{0} \mathcal{A}$ in the Quillen homotopy category of the $\mathcal{A}$-local model structure on $\mathfrak{M}^{\circ} \mathfrak{d}_{\mathcal{B}}$.

Using the theory reviewed above and the previous proposition, we can now prove Lemma 7.8.

Proof of Lemma 7.8 Fixing objects $x, y$ in $\mathcal{B}_{1}$, it suffices to show that the map $\mathcal{Q}_{\mathcal{A}_{1}}^{\mathcal{B}_{1}}(x, y) \rightarrow \mathcal{Q}_{\mathcal{A}_{2}}^{\mathcal{B}_{2}}(F x, F y)$ is a weak equivalence.

We take advantage of the functoriality of $\mathcal{Q}_{\mathcal{A}_{i}}^{\mathcal{B}_{i}}$ generalized to modules and the previous proposition. Choose an $\mathcal{A}_{1}$-local $\mathcal{B}_{1}$-module $\psi_{1}$ and an $\mathcal{A}_{1}$-local acyclic cofibration $q: \mathcal{B}_{1}(-, y) \rightarrow \psi_{1}$. It is clear from the characterization of the generating $\mathcal{A}_{1}$-local acyclic cofibrations that $q$ induces a weak equivalence $\mathcal{Q}_{\mathcal{A}_{1}}^{\mathcal{B}_{1}}(x, y) \rightarrow \mathcal{Q}_{\mathcal{A}_{1}}^{\mathcal{B}_{1}}\left(x, \psi_{1}\right)$. Moreover, since $\psi_{1}(a)$ is homotopically trivial for every object $a$ in $\mathcal{A}_{1}$, we have that the map $\psi_{1}(x) \rightarrow \mathcal{Q}_{\mathcal{A}_{1}}^{\mathcal{B}_{1}}\left(x, \psi_{1}\right)$ is a weak equivalence.

The functor $F^{*}$ from right $\mathcal{B}_{2}$-modules to right $\mathcal{B}_{1}$-modules has a left adjoint Lan $F$ defined by left Kan extension. Since $\operatorname{Lan}_{F}$ takes $\mathcal{B}_{1}(-, b)$ to $\mathcal{B}_{2}(-, F b)$ for any object $b$ in $\mathcal{B}_{1}$, Lan $_{F}$ takes the generating cofibrations and generating acyclic cofibrations of the $\mathcal{A}_{1}$-local model structure into the generating cofibrations and generating acyclic cofibrations of the $\mathcal{A}_{2}$-local model structure, ie, $\operatorname{Lan}_{F}, F^{*}$ is a Quillen adjunction on the local model structures. In particular, $\operatorname{Lan}_{F}$ takes $q$ to an $\mathcal{A}_{2}$-local acyclic cofibration $\mathcal{B}_{2}(-, F y) \rightarrow \operatorname{Lan}_{F} \psi_{1}$. Choose an $\mathcal{A}_{2}$-local object $\psi_{2}$ and an $\mathcal{A}_{2}$-local acyclic cofibration $\operatorname{Lan}_{F} \psi_{1} \rightarrow \psi_{2}$. Now we have weak equivalences

$$
\mathcal{Q}_{\mathcal{A}_{2}}^{\mathcal{B}_{2}}(F x, F y) \longrightarrow \mathcal{Q}_{\mathcal{A}_{2}}^{\mathcal{B}_{2}}\left(F x, \operatorname{Lan}_{F} \psi_{1}\right) \longrightarrow \mathcal{Q}_{\mathcal{A}_{2}}^{\mathcal{B}_{2}}\left(F x, \psi_{2}\right) .
$$

Moreover, since $\psi_{2}(a)$ is homotopically trivial for every object $a$ in $\mathcal{A}_{2}$, we have that the map $\psi_{2}(F x) \rightarrow \mathcal{Q}_{\mathcal{A}_{2}}^{\mathcal{B}_{2}}\left(F x, \psi_{2}\right)$ is a weak equivalence. 
Applying Proposition 7.11 and the hypothesis that $\mathcal{B}_{1} \rightarrow \mathcal{B}_{2}$ induces an embedding of $\pi_{0} \mathcal{B}_{1} / \pi_{0} \mathcal{A}_{1}$ into $\pi_{0} \mathcal{B}_{2} / \pi_{0} \mathcal{A}_{2}$, we see that $\psi_{1}(b) \rightarrow \psi_{2}(F b)$ is a weak equivalence for every object $b$ in $\mathcal{B}_{1}$. Thus, we have shown that in the commutative diagram

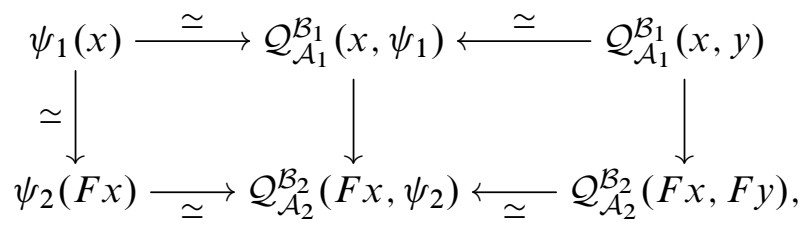

the arrows marked " $\simeq$ " are weak equivalences. It follows that the map $\mathcal{Q}_{\mathcal{A}_{1}}^{\mathcal{B}_{1}}(x, y) \rightarrow$ $\mathcal{Q}_{\mathcal{A}_{2}}^{\mathcal{B}_{2}}(F x, F y)$ is a weak equivalence.

\section{Applications of the general localization theorem}

We now turn to the applications of the general theory of the preceding sections to $T H H$ and $T C$ of schemes. We begin with a discussion of the spectral enrichment of the derived category of a scheme. Recent work of Dugger, Schwede and Shipley $[11 ; 12$; 38] shows that any stable category can be regarded as enriched in symmetric spectra and one approach would be to apply this theory in the setting of categories of unbounded complexes to construct a spectral derived category from first principles. On the other hand, such an approach would demand a comparison with the DG-category structures that arise in nature on categories of complexes. For this reason, we take the simpler approach of lifting DG-categories to associated spectral categories.

For a scheme $X$, let $\mathcal{K}^{\mathrm{DG}}(X)$ denote the pretriangulated DG-category of unbounded (cohomologically graded) complexes of sheaves of $\mathcal{O}_{X}$-modules; its homotopy category $\pi_{0} \mathcal{K}^{\mathrm{DG}}(X)$ is the triangulated category typically denoted $\mathcal{K}(X)$ of unbounded complexes and chain homotopy classes of maps. The derived category $\mathcal{D}(X)$ is the localization of $\mathcal{K}(X)$ obtained by inverting the quasi-isomorphisms. The derived category of perfect complexes $\mathcal{D}_{\text {parf }}(X)$ is the full triangulated subcategory of unbounded complexes locally quasi-isomorphic to strictly bounded complexes of vector bundles. By choosing a large enough cardinal $\aleph$ and restricting to the perfect complexes whose underlying sets are in $\boldsymbol{\aleph}$, we can find a small full pretriangulated subcategory $\mathcal{K}_{\text {parf }}^{\mathrm{DG}}(X)$ of $\mathcal{K}^{\mathrm{DG}}(X)$ consisting of perfect complexes and having the property that the triangulated quotient of the homotopy category by the full subcategory of acyclics is equivalent to $\mathcal{D}_{\text {parf }}(X)$ via the canonical map. Moreover, when $X$ is quasicompact and quasiseparated, the full subcategory $\mathcal{K}_{\text {parf }}^{\mathrm{DG}}(X)_{b}$ consisting of those complexes in $\mathcal{K}_{\text {parf }}^{\mathrm{DG}}(X)$ that are strictly bounded above and degreewise flat $\mathcal{O}_{X}$-modules also has the property that the triangulated quotient of the homotopy category by the full subcategory of acyclics is equivalent to $\mathcal{D}_{\text {parf }}(X)$ via the canonical map. 
Keller [25] and Drinfeld [10] described "quotient" DG-categories whose homotopy categories model the quotients of triangulated categories. We obtain small DGcategories $\mathcal{D}_{\text {parf }}^{\mathrm{DG}}(X)$ and (for $X$ quasicompact and quasiseparated) $\mathcal{D}_{\text {parf }}^{\mathrm{DG}}(X)_{b}$ whose homotopy categories are equivalent to the derived category $\mathcal{D}_{\text {parf }}$. We obtain small spectral categories $\mathcal{D}_{\text {parf }}^{S}(X)$ and $\mathcal{D}_{\text {parf }}^{S}(X)_{b}$ associated to $\mathcal{D}_{\text {parf }}^{\mathrm{DG}}(X)$ and $\mathcal{D}_{\text {parf }}^{\mathrm{DG}}(X)_{\mathrm{b}}$. We now prove Theorems 1.1, 1.3 and 1.4 from the introduction; in all cases, the results for $T R$ and $T C$ follow from the corresponding results for $T H H$ by Proposition 3.10.

Proof of Theorem 1.1 The theorem follows from Theorem 7.1: For the first statement, the Thomason-Trobaugh localization sequence, we apply Theorem 7.1 with $\mathcal{B}=$ $\mathcal{D}_{\text {parf }}^{S}(X), \mathcal{A}$ the full spectral subcategory of $\mathcal{D}_{\text {parf }}^{S}(X)$ consisting of those complexes that are supported on $X-U$, and $\mathcal{C}=\mathcal{D}_{\text {parf }}^{S}(U)$, using a lift $\mathcal{B} \rightarrow \mathcal{C}$ of the DG-functor $j^{*}: \mathcal{D}_{\text {parf }}^{\mathrm{DG}}(X) \rightarrow \mathcal{D}_{\text {parf }}^{\mathrm{DG}}(U)$. The Mayer-Vietoris statement follows from the localization statement and Corollary 5.7 since the inclusion in $X$ of any open set $V$ containing $X-U$ induces an equivalence on the derived categories of perfect complexes supported on $X-U=V-U \cap V$.

Proof of Theorem 1.3 We choose an affine open cover $\left\{U_{1}, \ldots, U_{r}\right\}$ of $X$. For each $i_{1}, \ldots, i_{n}$ let $U_{i_{1}, \ldots, i_{n}}=U_{i_{1}} \cap \cdots \cap U_{i_{n}}$ and let $A_{i_{1}, \ldots, i_{n}}=\mathcal{O}_{U_{i_{1}, \ldots, i_{n}}}$. Since $X$ is semiseparated, without loss of generality, $U_{i_{1}, \ldots, i_{n}}=\operatorname{Spec} A_{i_{1}, \ldots, i_{n}}$. We now construct a Čech complex on $T H H$ associated to this cover as follows.

Let $\mathcal{A}$ denote the full subcategory of $\mathcal{K}_{\text {parf }}^{\mathrm{DG}}(X)$ consisting of the acyclic complexes, and for each (nonempty) $U_{i_{1}, \ldots, i_{n}}$, let $\mathcal{A}_{i_{1}, \ldots, i_{n}}$ denote the full subcategory of $\mathcal{K}_{\text {parf }}^{\mathrm{DG}}(X)$ of objects acyclic on $U_{i_{1}, \ldots, i_{n}}$. We have defined $\mathcal{D}_{\text {parf }}^{\mathrm{DG}}(X)$ by Drinfeld's quotient category construction $\mathcal{K}_{\text {parf }}^{\mathrm{DG}}(X) / \mathcal{A}$. For all $i_{1}, \ldots, i_{n}$ and $U=U_{i_{1}} \cap \cdots \cap U_{i_{n}}$, the DGfunctor $j^{*}: \mathcal{K}_{\text {parf }}^{\mathrm{DG}}(X) \rightarrow \mathcal{K}_{\text {parf }}^{\mathrm{DG}}(U)$ (associated to $j: U \subset X$ ) induces a DG-functor $\mathcal{K}_{\text {parf }}^{\mathrm{DG}}(X) / \mathcal{A}_{i_{1}, \ldots, i_{n}} \rightarrow \mathcal{D}_{\text {parf }}^{\mathrm{DG}}(U)$ that is a DG-equivalence onto its image. Moreover, this functor is cofinal in that every perfect complex on $U$ is a direct summand of $j^{*}$ of a perfect complex on $X$. We apply functorial factorization to construct the associated (fibrant) spectral categories $\mathcal{D}_{i_{1}, \ldots, i_{n}}$. This constructs a strictly commuting diagram of spectral functors associated to intersections of the open sets in the cover. Moreover, comparing this construction with the argument above for Theorem 1.1, we see that the map

$$
\operatorname{THH}\left(\mathcal{D}_{\text {parf }}^{S}(X)\right) \longrightarrow \operatorname{holim}_{\mathcal{S}_{r}} \operatorname{THH}\left(\mathcal{D}_{i_{1}, \ldots, i_{n}}\right)
$$

is a weak equivalence, where $\mathcal{S}$ is the partially ordered set of nonempty subsets of $1, \ldots, r$.

Each of the categories $\mathcal{D}_{i_{1}}, \ldots, i_{n}$ has an object called $\mathcal{O}_{X}$ whose endomorphism spectrum is an Eilenberg-Mac Lane ring spectrum for $A_{i_{1}, \ldots, i_{n}}$. Write $H A_{i_{1}, \ldots, i_{n}}$ for 
$\mathcal{D}_{i_{1}, \ldots, i_{n}}\left(\mathcal{O}_{X}, \mathcal{O}_{X}\right)$. Since the objects called $\mathcal{O}_{X}$ in $\mathcal{D}_{i_{1}, \ldots, i_{n}}$ are compatible under inclusion of intersections, we obtain a map

$$
\operatorname{holim}_{\mathcal{S}_{r}} \operatorname{THH}\left(H A_{i_{1}, \ldots, i_{n}}\right) \longrightarrow \operatorname{holim}_{\mathcal{S}_{r}} \operatorname{THH}\left(\mathcal{D}_{i_{1}, \ldots, i_{n}}\right) .
$$

The lefthand spectrum is easily seen to be equivalent to the Čech cohomology associated to the cover $\left\{U_{1}, \ldots, U_{r}\right\}$ of the Zariski presheaf of symmetric spectra $\operatorname{THH}\left(\mathcal{O}_{(-)}\right)$. Geisser and Hesselholt [16, 3.2.1] showed that the homotopy groups of THH of a commutative ring form a quasicoherent sheaf, and so the lefthand homotopy limit computes the hypercohomology spectrum of $\operatorname{THH}\left(\mathcal{O}_{(-)}\right)$, ie, $\operatorname{TH} H(X)$ as defined by $[16,3.2 .3]$.

Thus, Theorem 1.3 for quasicompact semiseparated schemes reduces to showing that each map $\operatorname{TH} H\left(H A_{i_{1}, \ldots, i_{n}}\right) \rightarrow \operatorname{THH}\left(\mathcal{D}_{i_{1}, \ldots, i_{n}}\right)$ is a weak equivalence. This follows from Theorem 5.12.

Proof of Theorem 1.4 We have functors

$$
\begin{aligned}
& L i^{*}: \mathcal{D}_{\text {parf }}^{\mathrm{DG}}(X)_{b} \longrightarrow \mathcal{D}_{\text {parf }}^{\mathrm{DG}}\left(X^{\prime}\right)_{\mathrm{b}}, \\
& L j^{*}: \mathcal{D}_{\text {parf }}^{\mathrm{DG}}(Y)_{b} \longrightarrow \mathcal{D}_{\text {parf }}^{\mathrm{DG}}\left(Y^{\prime}\right)_{b} .
\end{aligned}
$$

Each of these is a DG-equivalence to its image. Let $\mathcal{B}_{1}=\mathcal{D}_{\text {parf }}^{S}\left(X^{\prime}\right)_{\mathrm{b}}$ and $\mathcal{B}_{2}=$ $\mathcal{D}_{\text {parf }}^{S}\left(Y^{\prime}\right)_{b}$, and let $\mathcal{A}_{1}$ denote the full spectral subcategory of $\mathcal{B}_{1}$ consisting of objects equivalent to those in the image of $L i^{*}$ and $\mathcal{A}_{2}$ denote the full spectral subcategory of $\mathcal{B}_{2}$ of objects in the image of $L j^{*}$. The map $L p^{*}$ lifts to a map $\mathcal{D}_{\text {parf }}^{S}(X)_{b} \rightarrow \mathcal{D}_{\text {parf }}^{S}\left(X^{\prime}\right)_{b}$ that lands in $\mathcal{A}_{1}$ and is a DK-equivalence to $\mathcal{A}_{1}$. Likewise, $L q^{*}$ induces a DK-equivalence of $\mathcal{D}_{\text {parf }}^{S}\left(Y^{\prime}\right)$ with $\mathcal{A}_{2}$. In this way, we obtain a strictly commuting spectral model for the DG-functors $L p^{*}, L q^{*}, L i^{*}$ and $L j^{*}$ as a map of localization pairs $\left(\mathcal{B}_{1}, \mathcal{A}_{1}\right) \rightarrow\left(\mathcal{B}_{2}, \mathcal{A}_{2}\right)$. By [42, Section $\left.2.7 ; 8,1.5\right]$, this map induces an equivalence on quotient triangulated categories, and therefore a weak equivalence $\operatorname{CTHH}\left(\mathcal{B}_{1} / \mathcal{A}_{1}\right) \rightarrow \operatorname{CTHH}\left(\mathcal{B}_{2} / \mathcal{A}_{2}\right)$ by Theorem 7.2. Corollary 7.3 gives the corresponding result on $T C$. Theorem 1.4 now follows.

Proof of Theorem 1.5 Let $\pi: \mathbb{P} \mathcal{E}_{X} \rightarrow X$ be the projective bundle of an algebraic vector bundle $\mathcal{E}$ of rank $r$. Thomason [42, Section 2.7] constructed a triangulated filtration

$$
0 \simeq \mathcal{A}_{r} \subset \mathcal{A}_{r-1} \subset \cdots \subset \mathcal{A}_{0}=\mathcal{D}_{\text {parf }}\left(\mathbb{P} \mathcal{E}_{X}\right)
$$

of the derived category as follows. Let $\mathcal{A}_{k}$ denote the full subcategory of $\mathcal{D}_{\text {parf }}\left(\mathbb{P} \mathcal{E}_{X}\right)$ consisting of complexes $Z$ such that $R \pi_{*}\left(Z \otimes \mathcal{O}_{\mathbb{P} \mathcal{E}_{X}}(i)\right)=0$ for $0 \leq i<k$. By definition, $\mathcal{A}_{0}=\mathcal{D}_{\text {parf }}^{\mathrm{DG}}(X)\left(\mathbb{P} \mathcal{E}_{X}\right)$, and since $\mathcal{E}$ is rank $r, \mathcal{A}_{r}$ consists of acyclic complexes $[42,2.5]$. Furthermore, $\mathcal{A}_{k}$ admits the alternate description as the thick 
subcategory of $\mathcal{D}_{\text {parf }}\left(\mathbb{P} \mathcal{E}_{X}\right)$ generated by $L \pi^{*}(-) \otimes \mathcal{O}_{\mathbb{P} \mathcal{E}_{X}}(-j)$ for $k \leq j<r$ (see also $[8,1.2])$. Let $\mathcal{A}_{r-1}^{\prime}, \ldots, \mathcal{A}_{0}^{\prime}$ denote the corresponding filtration on $\mathcal{D}_{\text {parf }}^{S}\left(\mathbb{P} \mathcal{E}_{X}\right)$.

The functor $L \pi^{*}(-) \otimes \mathcal{O}_{\mathbb{P} \mathcal{E}_{X}}(-k)$ from $\mathcal{D}_{\text {parf }}(X)$ to $\mathcal{A}_{k}$ admits a refinement to a DGfunctor $\mathcal{D}_{\text {parf }}^{\mathrm{DG}}(X) \rightarrow \mathcal{D}_{\text {parf }}^{\mathrm{DG}}\left(\mathbb{P} \mathcal{E}_{X}\right)$, which we can lift to a spectral functor $\mathcal{D}_{\text {parf }}^{S}(X) \rightarrow \mathcal{A}_{k}^{\prime}$. Viewed as map of localization pairs $\left(\mathcal{D}_{\text {parf }}^{S}(X), 0\right) \rightarrow\left(\mathcal{A}_{k}^{\prime}, \mathcal{A}_{k+1}^{\prime}\right)$, the induced map of triangulated quotients $\mathcal{D}_{\text {parf }}(X) \rightarrow \mathcal{A}_{k} / \mathcal{A}_{k+1}$ is an equivalence [42, Section 2.7]. Theorem 7.2 then shows that the induced map $\operatorname{THH}(X) \rightarrow \operatorname{CTHH}\left(\mathcal{A}_{k}^{\prime}, \mathcal{A}_{k+1}^{\prime}\right)$ is a weak equivalence. In particular, we obtain split cofibration sequences

$$
\operatorname{THH}\left(\mathcal{A}_{k+1}^{\prime}\right) \longrightarrow \operatorname{THH}\left(\mathcal{A}_{k}^{\prime}\right) \longrightarrow \operatorname{THH}(X),
$$

and hence weak equivalences

$$
\operatorname{THH}\left(\mathcal{A}_{k}^{\prime}\right) \simeq \operatorname{THH}\left(\mathcal{A}_{k+1}^{\prime}\right) \times T H H(X) .
$$

for $k=0, \ldots, r-1$. Using Corollary 7.3, we get the corresponding results for $T C$. This completes the proof of Theorem 1.5.

\section{The cyclotomic trace from $K^{B}$}

In this section we show that the cyclotomic trace from $K$-theory to $T H H$ and $T C$ factors through Thomason and Trobaugh's construction of Bass' nonconnective $K$-theory $K^{B}$ [43, Section 6]. Using a version of Bass' fundamental theorem for $T H H$, we factor the cyclotomic trace map from connective $K$-theory on affine schemes (commutative rings) through $K^{B}$. This factorization holds more generally for maps from $K$-theory to any theory satisfying the appropriate analogue of Bass' fundamental theorem, and is natural for such functors to a (strict point-set) category of spectra. Since the trace map admits a model in which it is a map of presheaves restricted to affine covers, we obtain the factorization $K^{B} \rightarrow T H H$ on the level of presheaves, which we show lifts to a map of presheaves $K^{B} \rightarrow T C$. For quasicompact semiseparated schemes, $K^{B}$ is equivalent to the Čech hypercohomology spectrum of its presheaf by $[43,8.4]$. The work of the previous section (and [16]) shows that for such schemes $T H H$ and $T C$ are each equivalent to both the hypercohomology spectrum and Čech hypercohomology spectrum of their respective presheaves. This then constructs the trace map $K^{B} \rightarrow T C$ for all quasicompact, semiseparated schemes.

We begin by discussing the analogue of Bass' fundamental theorem that we need. For the purposes of this section, we say that a covariant functor $F$ from commutative rings to some (point-set) category of spectra is a Bass functor when it comes with a natural transformation $\tau: \Sigma F(R) \rightarrow F\left(R\left[t, t^{-1}\right]\right)$ and satisfies the following: 
(i) For any $R, F(R)$ is connective $\left(\pi_{n} F(R)=0\right.$ for $\left.n<0\right)$.

(ii) For any $R$ and any $n \geq 0$, the sequence

$$
0 \longrightarrow \pi_{n} F(R) \longrightarrow \pi_{n} F(R[t]) \oplus \pi_{n} F\left(R\left[t^{-1}\right]\right) \longrightarrow \pi_{n} F\left(R\left[t, t^{-1}\right]\right)
$$

induced by the inclusion maps is exact.

(iii) For any $R$ and any $n>0$, the composite map

$$
\begin{aligned}
\pi_{n-1} F(R)=\pi_{n} \Sigma F(R) & \longrightarrow \pi_{n} F\left(R\left[t, t^{-1}\right]\right) \\
& \longrightarrow \operatorname{Coker}\left(\pi_{n} F(R[t]) \oplus \pi_{n} F\left(R\left[t^{-1}\right]\right) \longrightarrow \pi_{n} F\left(R\left[t, t^{-1}\right]\right)\right)
\end{aligned}
$$

induced by $\tau$ is an isomorphism.

A map of Bass functors is a natural transformation $F \rightarrow G$ that commutes with the maps $\tau$. The key fact we need to apply this theory is the following (well-known) theorem, whose proof we review at the end of this section.

Theorem 9.1 The $K$-theory functor and the $T H H$ functor admit models that are Bass functors with the trace map a map of Bass functors.

As an immediate consequence of the definition, a Bass functor in particular comes with a natural 4-term exact sequences

$0 \longrightarrow \pi_{n} F(R) \rightarrow \pi_{n} F(R[t]) \oplus \pi_{n} F\left(R\left[t^{-1}\right]\right) \rightarrow \pi_{n} F\left(R\left[t, t^{-1}\right]\right) \rightarrow \pi_{n-1} F(R) \longrightarrow 0$

for $n>0$ with the map $\pi_{n} F\left(R\left[t, t^{-1}\right]\right) \rightarrow \pi_{n-1} F(R)$ naturally split. This exact sequence and splitting are functorial in maps of Bass functors. Bass' construction extends these sequences to all $n$ :

Definition 9.2 (Bass' construction) For a Bass functor $F$, let $\beta_{n} F=\pi_{n} F$ and let

$$
\tau_{n}: \beta_{n} F(R) \longrightarrow \beta_{n+1} F\left(R\left[t, t^{-1}\right]\right)
$$

be the map induced by $\tau$ for $n \geq 0$. Inductively, for $n \leq 0$, define

$$
\beta_{n-1} F(R)=\operatorname{Coker}\left(\beta_{n} F(R[x]) \oplus \beta_{n} F\left(R\left[x^{-1}\right]\right) \longrightarrow \beta_{n} F\left(R\left[x, x^{-1}\right]\right)\right)
$$


and $\tau_{n}: \beta_{n-1} F(R) \rightarrow \beta_{n} F\left(R\left[t, t^{-1}\right]\right)$ to be the induced map on cokernels

$$
\operatorname{Coker}\left(\begin{array}{c}
\beta_{n} F(R[x]) \\
\oplus \\
\beta_{n} F\left(R\left[x^{-1}\right]\right) \\
\downarrow \\
\beta_{n} F\left(R\left[x, x^{-1}\right]\right)
\end{array}\right) \rightarrow \operatorname{Coker}\left(\begin{array}{c}
\beta_{n+1} F\left(R\left[x, t, t^{-1}\right]\right) \\
\oplus \\
\beta_{n+1} F\left(R\left[x^{-1}, t, t^{-1}\right]\right) \\
\downarrow \\
\beta_{n+1} F\left(R\left[x, x^{-1}, t, t^{-1}\right]\right)
\end{array}\right)
$$

Applied to the $K$-theory functor, Bass' construction defines Bass' negative $K$-groups. Applied to the $T H H$ functor, $\beta_{n} T H H=0$ for $n<0$ since the map

$$
\pi_{0} T H H(R[x]) \oplus \pi_{0} T H H\left(R\left[x^{-1}\right]\right) \longrightarrow \pi_{0} T H H\left(R\left[x, x^{-1}\right]\right)
$$

is surjective. (It is the map $R[x] \oplus R\left[x^{-1}\right] \rightarrow R\left[x, x^{-1}\right]$.) Thomason and Trobaugh extended Bass' construction to a construction on spectra suitable for application to general Bass functors as defined above. The following is essentially a simplification of $[43,6.3]$.

Lemma 9.3 Let $F$ be a Bass functor. There exists a functor $F^{B}$ from commutative rings to spectra and a natural transformation $F \rightarrow F^{B}$ that is an isomorphism on $\pi_{n}$ for $n \geq 0$ and induces (as indicated in [43, 6.3]) a natural isomorphism $\beta_{n} F \rightarrow \pi_{n} F^{B}$ for $n<0$. The functor and natural transformation are functorial in maps of Bass functors.

We need a few of the details of the construction. Thomason and Trobaugh construct $F^{B}$ as the homotopy colimit of a sequence of functors

$$
F=F_{0}=F_{0}^{\prime} \longrightarrow F_{-1}^{\prime} \longrightarrow F_{-2}^{\prime} \longrightarrow \cdots F_{-k}^{\prime} \longrightarrow \cdots .
$$

The functor $F_{-k-1}^{\prime}$ is formed inductively as a homotopy pushout

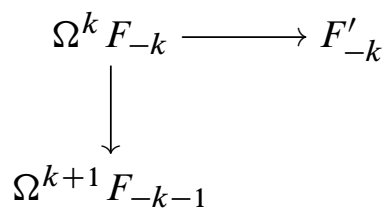

for functors $F_{-k}$ which come with natural transformations $\Sigma F_{-k} \rightarrow F_{-k-1}$. The functor $F_{-k-1}$ is defined inductively as the homotopy cofiber of the natural map

$$
F_{-k}(R[x]) \stackrel{h}{~}_{F_{-k}(R)} F_{-k}\left(R\left[x^{-1}\right]\right) \longrightarrow F_{-k}\left(R\left[x, x^{-1}\right]\right)
$$

(where $\cup^{h}$ means the homotopy pushout). The map $\Sigma F_{-k} \rightarrow F_{-k-1}$ comes from the 
canonical map $\Sigma F_{-k}(R) \rightarrow F_{-k}\left(R\left[t, t^{-1}\right]\right)$, constructed just as in Bass' construction in algebra, as the induced map on cofibers coming from the natural commutative diagram

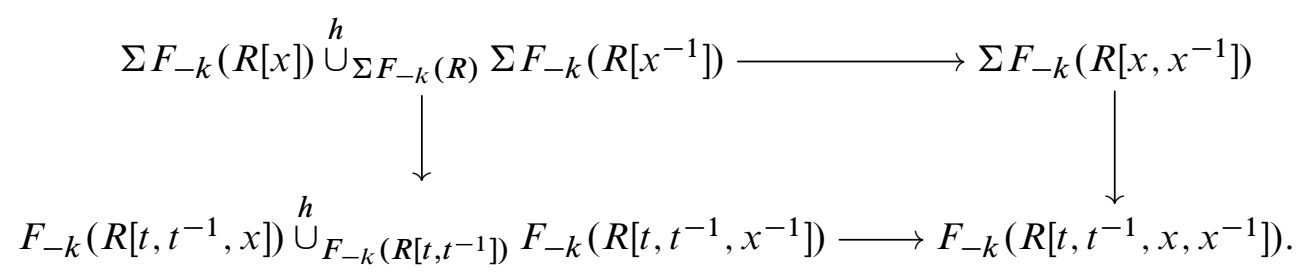

Our notation differs slightly from that of [43, 6.3]; our $F_{-k}$ is their $\Sigma^{k} F^{-k}$. As a consequence of this construction, we get the following observation.

Proposition 9.4 If $F$ is a Bass functor and factors through cyclotomic spectra (with $\tau$ a natural map of cyclotomic spectra), then the functor $F^{B}$ factors through cyclotomic spectra and the natural transformation $F \rightarrow F^{B}$ is a natural transformation of cyclotomic spectra.

Combining the Thomason-Trobaugh lemma with Theorem 9.1, we get a natural transformation of functors

$$
K^{B} \longrightarrow T H H^{B} \simeq T H H .
$$

The model of $T H H$ constructed below and referred to in Theorem 9.1 satisfies the hypotheses of Proposition 9.4. Then $T H H^{B}$ is a cyclotomic spectrum and we can form a functor $T C$ as the appropriate limit (or pro-object). We obtain the following commutative diagram of functors.

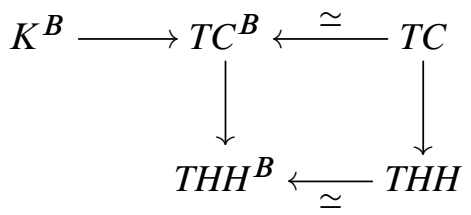

This extends the trace to nonconnective $K$-theory.

The remainder of the section proves Theorem 9.1. We begin by observing that $T H H$ algebraically satisfies the analogue of Bass' fundamental theorem. For a commutative ring $R$, the Eilenberg Mac Lane spectrum $H R$ is a commutative ring spectrum in any of the modern categories of spectra. We have a weak equivalence of associative ring spectra $H R \wedge T S \rightarrow H R[t]$, where $T S$ is the free associative ring spectrum on the sphere spectrum (or a cofibrant model of it). We then get weak equivalences 
of $\operatorname{THH}(R)-$ modules

$T H H(R[t]) \simeq T H H(R \wedge T S) \simeq T H H(R) \wedge T H H(T S)$

$\cong T H H(R) \wedge_{H R}(H R \wedge T H H(T S))$.

We also have the identifications

$$
H R \wedge T H H(T S) \cong T H H^{H R}(H R \wedge T S) \simeq T H H^{H R}(R[t]),
$$

where $T H H^{H R}(R[t])$ is as defined in [14, Section IX.1.7] and is essentially the spectrum whose homotopy groups are $H H_{*}^{R}(R[t])$. Since this Hochschild homology is a free module over $R$, we obtain the computation

$$
\pi_{*} T H H(R[t]) \cong \pi_{*} T H H(R) \otimes_{R} H H_{*}^{R}(R[t]) \cong \pi_{*} T H H(R) \otimes_{R} R[t]\left\langle 1, \sigma_{t}\right\rangle,
$$

where 1 is in degree zero and $\sigma_{t}$ is in degree one. This is an isomorphism of $\pi_{*} T H H(R)$-modules, and is natural in $R$ and $T S$, though not obviously in $R[t]$.

Writing $T S\left[t^{-1}\right]$ for the localization of $T S$ under multiplication by the generator of $\pi_{0} S$ (which we are thinking of as $t$ ), we have a weak equivalence of associative ring spectra $H R \wedge T S\left[t^{-1}\right] \rightarrow H R\left[t, t^{-1}\right]$, and as above, we get the weak equivalences

$$
T H H\left(R\left[t, t^{-1}\right]\right) \simeq T H H\left(R \wedge T S\left[t^{-1}\right]\right) \simeq T H H(R) \wedge_{H R} T H H^{H R}\left(H R\left[t, t^{-1}\right]\right)
$$

and the computation

$$
\begin{aligned}
\pi_{*} T H H\left(R\left[t, t^{-1}\right]\right) & \cong \pi_{*} T H H(R) \otimes_{R} H H_{*}^{R}\left(R\left[t, t^{-1}\right]\right) \\
& \cong \pi_{*} T H H(R) \otimes_{R} R\left[t, t^{-1}\right]\left\langle 1, \sigma_{t}\right\rangle,
\end{aligned}
$$

Again, this is an isomorphism of $\pi_{*} T H H(R)$-modules and is natural in $R$ and $T S\left[t^{-1}\right]$, though not obviously in $R\left[t, t^{-1}\right]$.

The map $R[x] \rightarrow R\left[t, t^{-1}\right]$ sending $x$ to $t^{-1}$ is induced by a map of associative ring spectra $T S \rightarrow T S\left[t^{-1}\right]$, namely, the map induced by the map $S \rightarrow T S\left[t^{-1}\right]$ sending the generator of $\pi_{0} S$ to $t^{-1}$ in $\pi_{0} T S\left[t^{-1}\right] \cong \mathbb{Z}\left[t, t^{-1}\right]$. Thus, we can compute the maps in Bass' sequence for $\pi_{*} T H H$ in terms of Hochschild homology. This then becomes an easy computation with resolutions: the inclusion $R\left[t^{-1}\right] \rightarrow R\left[t, t^{-1}\right]$ induces the map of $\pi_{*} T H H(R) \otimes_{R} R\left[t^{-1}\right]$-modules that sends 1 to 1 and $\sigma_{t^{-1}}$ to $-t^{-2} \sigma_{t}$. It follows that the sequence of graded abelian groups

$$
0 \longrightarrow \pi_{*} T H H(R) \longrightarrow \pi_{*} T H H(R[t]) \oplus \pi_{*} T H H\left(R\left[t^{-1}\right]\right) \longrightarrow \pi_{*} T H H\left(R\left[t, t^{-1}\right]\right)
$$

is exact and the map $\pi_{*-1} T H H(R) \rightarrow \pi_{*} T H H\left(R\left[t, t^{-1}\right]\right)$ induced by the inclusion and multiplication by $t^{-1} \sigma_{t}$ induces an isomorphism from $\pi_{*-1} T H H(R)$ onto the cokernel of the last map above. 
Thomason and Trobaugh [43, Section 6] prove an analogous formulation of Bass' fundamental theorem for $K$-theory: The three term sequence is exact and the map $K_{n-1} R \rightarrow K_{n} R\left[t, t^{-1}\right]$ induced by the inclusion and multiplication by $t$ (viewed as an element of $K_{1} R\left[t, t^{-1}\right]$ ) induces an isomorphism onto the cokernel for $n>1$. Since the Dennis trace map takes the element $t$ in $K_{1}\left(\mathbb{Z}\left[t, t^{-1}\right]\right)$ to the element $t \sigma_{t^{-1}}=-t^{-1} \sigma_{t}$ in $H H_{1}\left(\mathbb{Z}\left[t, t^{-1}\right]\right)$, multiplication by the image of $t$ under the trace to $T H H$ also provides an isomorphism from $\pi_{*-1} T H H(R)$ to the cokernel for $\pi_{*} T H H$. We now have what we need to prove Theorem 9.1.

Proof of Theorem 9.1 We give an argument that uses minimal details of the construction of the cyclotomic trace. The next section contains a review of the cyclotomic trace and a more direct construction of the extension to nonconnective $K$-theory. For this argument, we use the model of the trace map described by Dundas and McCarthy [13, Section 2.1.6], with some of the modifications of Geisser and Hesselholt [16, Section 6.3] that involve the multiplicative structure. We regard $K$ as a functor from exact categories to symmetric spectra, and we regard $T H$ (in the notation of [16, Section 6.3]) as a functor from exact categories to symmetric spectra of cyclotomic spectra. The point-set category of spectra we work in is the category of symmetric spectra of orthogonal spectra, and we use the free functor in the orthogonal spectrum direction $F_{0}^{\mathcal{I} \varphi}$ (and geometric realization) to convert symmetric spectra to symmetric spectra of orthogonal spectra.

Since $K$ and $T H$ are functors on exact categories, to get functors on commutative rings, we need a model of the exact category of finitely generated projective modules that is strictly functorial in maps of commutative rings. For this, consider the category $\mathcal{P}(R)$ whose objects are pairs $(P, m)$ where $P$ is a projective submodule of $R^{m}$, and whose maps $(P, m) \rightarrow(Q, n)$ are the $R$-module maps $P \rightarrow Q$. This is an exact category in the evident way. A map of rings $R \rightarrow R^{\prime}$ induces a map $\mathcal{P}(R) \rightarrow \mathcal{P}\left(R^{\prime}\right)$ by extension of scalars and the canonical identification $R^{\prime} \otimes_{R} R^{m} \cong R^{\prime m}$; this makes $\mathcal{P}$ a functor from commutative rings to exact categories. Defining $K(R)=K(\mathcal{P}(R))$ and $T H H(R)=$ $T H(\mathcal{P}(R)$ ) (in the notation of [16]), we obtain functors from commutative rings to symmetric spectra and from commutative rings to symmetric spectra of cyclotomic spectra, respectively. We also obtain a natural transformation of symmetric spectra of orthogonal spectra $F_{0}^{\mathcal{I S}} F_{0} K(R) \rightarrow T H H(R)$.

We have a biexact strictly associative tensor product on $\mathcal{P}(R)$ defined by the usual tensor product and the (lexicographical order) identification $R^{m} \otimes_{R} R^{n} \cong R^{m n}$. As observed in [16, Section 6.3], it follows that $K(R)$ is naturally an associative ring symmetric spectrum. Moreover, using the associative smash product pairing of a space and a cyclotomic spectrum, $T H H(R)$ becomes a module over $K(R)$ in the category of 
symmetric spectra of cyclotomic spectra. If it were possible, what we would like to do as a next step is choose a map $\bar{t}$ from $S^{1}$ to the zeroth space of $K\left(\mathbb{Z}\left[t, t^{-1}\right]\right)$ representing the element $t$ of $K_{1}\left(\mathbb{Z}\left[t, t^{-1}\right]\right)$. We would then get natural point-set maps of symmetric spectra $\tau: \Sigma K(R) \rightarrow K\left(R\left[t, t^{-1}\right]\right)$ using the inclusions and multiplication

$$
K(R) \wedge K\left(\mathbb{Z}\left[t, t^{-1}\right]\right) \longrightarrow K\left(R\left[t, t^{-1}\right]\right) \wedge K\left(R\left[t, t^{-1}\right]\right) \longrightarrow K\left(R\left[t, t^{-1}\right]\right) .
$$

However, although $t$ is represented in the first space of $K\left(\mathbb{Z}\left[t, t^{-1}\right]\right)$, it is not represented in the zeroth space. To fix this, we use a variant of the functor $M$ of $[39,3.2 .1]$.

For a symmetric spectrum $X$, let $M X$ be the symmetric spectrum of topological spaces

$$
M X=\operatorname{hocolim}_{\mathbf{n} \in \mathcal{I}} \Omega^{n}\left|s h_{n} X\right|
$$

where $s h_{n} X$ is the symmetric spectrum $s h_{n} X_{k}=X_{k+n}$ [24, 2.2.12]. The inclusion of $X$ as $\Omega^{0} s h_{0} X$ induces a natural transformation $X \rightarrow M X$. When $X$ is a positive $\Omega$-spectrum like $K(R), M X$ is an $\Omega$-spectrum and $X \rightarrow M X$ is a positive level equivalence; $\operatorname{cf}[39$, page 168]. Moreover, $M$ is a monoidal functor with

$M X \wedge M Y \cong \operatorname{hocolim}_{(\mathbf{m}, \mathbf{n}) \in \mathcal{I}^{2}}\left(\Omega^{m}\left|s h_{m} X\right| \wedge \Omega^{n}\left|s h_{n} Y\right|\right)$

$$
\longrightarrow \operatorname{hocolim}_{\mathbf{p} \in \mathcal{I}} \Omega^{p}\left(\left|s h_{p}(X \wedge Y)\right|\right)=M(X \wedge Y)
$$

induced by disjoint union functor $\mathcal{I} \times \mathcal{I} \rightarrow \mathcal{I}$, the map

$$
\Omega^{m}\left|X_{m+k}\right| \wedge \Omega^{n}\left|Y_{n+\ell}\right| \longrightarrow \Omega^{m+n}\left|X_{m+k} \wedge Y_{n+\ell}\right| \longrightarrow \Omega^{m+n}\left|(X \wedge Y)_{m+k+n+\ell}\right|,
$$

and the appropriate permutation $(X \wedge Y)_{m+k+n+\ell} \cong(X \wedge Y)_{m+n+k+\ell}$ on the $(m+n+k+\ell)$-th space of $X \wedge Y$. We have the analogous endofunctor $M$ and natural transformation $X \rightarrow M X$ on the category of symmetric spectra of cyclotomic spectra. The cyclotomic spectra $T H H(R)$ are $\Omega$-spectra in the symmetric spectrum direction, and so the map $\operatorname{THH}(R) \rightarrow \operatorname{MTHH}(R)$ is a level equivalence in the symmetric spectrum direction.

Now we choose a point-set map $\bar{t}$ from $S^{1}$ to the zeroth space of $M K\left(\mathbb{Z}\left[t, t^{-1}\right]\right)$. We define $\tau: \Sigma M K(R) \rightarrow M K\left(R\left[t, t^{-1}\right]\right)$ to be the natural transformation of symmetric spectra induced by multiplication with the point-set representative $\bar{t}$ of $t$. Likewise, we define $\tau: \Sigma M T H H(R) \rightarrow M T H H\left(R\left[t, t^{-1}\right]\right)$ to be the natural transformation of symmetric spectra of cyclotomic spectra induced by multiplication by $\bar{t}$. Using $F_{0}^{\mathcal{I} \mathscr{S}} M K(R)$ and $M T H H(R)$ as our models for $K(R)$ and $T H H(R)$, this constructs $K$ and $T H H$ as Bass functors, with $T H H$ a Bass functor in a point-set category of cyclotomic spectra, and the cyclotomic trace a natural transformation of Bass functors. 


\section{The cyclotomic trace for DG-Waldhausen categories}

In Section 8 , we implicitly constructed the cyclotomic trace connecting the $K$-theory of a scheme to the $T C$ and $T H H$ of the associated spectral derived category via the comparison to the Geisser-Hesselholt definition of these spectra in terms of hypercohomology. This streamlined approach allowed us to avoid the lengthy technical development necessary for a more intrinsic construction of the cyclotomic trace, and was sufficient for our applications. In this section, we complete the theory of $T C$ and $\mathrm{THH}$ of spectral derived categories by describing an intrinsic construction of the cyclotomic trace.

Our construction of the cyclotomic trace follows the perspective of [13, Section 2.1.6] that the trace should be regarded as "the inclusion of the objects" from a Waldhausen category to a model of $T H H$ which mixes the cyclic bar construction and Waldhausen's $S$ • construction. In order to enable this mixing, we work with a class of Waldhausen categories equipped with a DG-enrichment that is compatible with the Waldhausen structure. We call these DG-Waldhausen categories; they are in particular complicial bi-Waldhausen categories [43, 1.2.11].

Definition 10.1 A DG-Waldhausen category consists of a small full subcategory $\mathcal{C}$ of the category of complexes of an abelian category $A b_{\mathcal{C}}$ (which is part of the structure), and a subcategory $w \mathcal{C}$ of $\mathcal{C}$ called the weak equivalences, satisfying the following properties.

(i) $\mathcal{C}$ contains zero.

(ii) $\mathcal{C}$ is closed under pushouts along degreewise split monomorphisms and pullbacks along degreewise split epimorphisms.

(iii) $\mathcal{C}$ is closed under cones and cocones.

(iv) The weak equivalences contain the quasi-isomorphisms of complexes, are preserved by pushout along degreewise-split monomorphisms and pullback along degreewise-split epimorphisms, and satisfy Waldhausen's saturation and extension properties.

A $D G$-exact functor from $\left(\mathcal{C}, A b_{\mathcal{C}}, w \mathcal{C}\right)$ to $\left(\mathcal{C}^{\prime}, A b_{\mathcal{C}^{\prime}}, w \mathcal{C}^{\prime}\right)$ is an additive functor $A b_{\mathcal{C}} \rightarrow$ $A b_{\mathcal{C}^{\prime}}$ that takes $\mathcal{C}$ into $\mathcal{C}^{\prime}$ and $w \mathcal{C}$ into $w \mathcal{C}^{\prime}$.

By abuse of language, we usually call $\mathcal{C}$ the DG-Waldhausen category. In the definition, the cone $C X$ and cocone $C^{\prime} X$ of a complex $X$ are the usual contractible complexes 
that fit into the short exact sequences

$$
\begin{gathered}
0 \longrightarrow X \longrightarrow C X \longrightarrow X[1] \longrightarrow 0 \\
0 \longrightarrow X[-1] \longrightarrow C^{\prime} X \longrightarrow X \longrightarrow 0 .
\end{gathered}
$$

Waldhausen's saturation property on the weak equivalences means that $w \mathcal{C}$ satisfies "two-out-of-three": for composable maps $f, g$ in $\mathcal{C}$, if any two of the maps $f, g$, and $g \circ f$ are in $w \mathcal{C}$ then so is the third. Waldhausen's extension property means that when

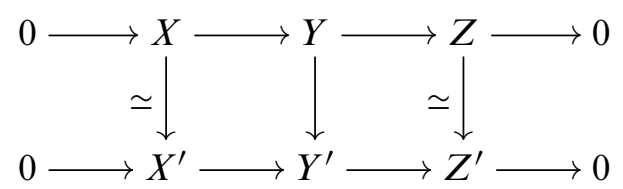

is a commutative diagram of degree-wise split short exact sequences with the maps $X \rightarrow X^{\prime}$ and $Z \rightarrow Z^{\prime}$ in $w \mathcal{C}$, then the map $Y \rightarrow Y^{\prime}$ is in $w \mathcal{C}$. As a consequence, the subcategory $\mathcal{C}^{w}$ of $w \mathcal{C}$-acyclic objects (those objects weakly equivalent to 0 ) is closed under extensions; the extension property is equivalent to this closure condition.

A DG-Waldhausen category obtains the structure of a pretriangulated DG-category with the usual mapping complexes and also the structure of a Waldhausen category (in fact a complicial bi-Waldhausen category) with the cofibrations the degreewise-split monomorphisms. Therefore we can construct both its algebraic $K$-theory (using the Waldhausen category structure), as well as its $T H H$ and $T C$ (lifting the DG-category structure to an associated spectral category structure). The weak equivalences of the Waldhausen category structure specify additional homotopical data beyond that in the mapping spectra: The natural homotopy category of the Waldhausen category structure is the localization of the homotopy category associated to the DG-category obtained by localizing with respect to the weak equivalences. In the terminology of Section 7, this homotopy category is the triangulated quotient of $\mathcal{C}$ by the subcategory $\mathcal{C}^{w}$ of $w \mathcal{C}$-acyclics. Thus, the proper notion of $T H H$ and $T C$ are the $T H H$ and $T C$ of the localization pair, $\operatorname{CTHH}\left(\mathcal{C} / \mathcal{C}^{w}\right)$ and $C T C\left(\mathcal{C} / \mathcal{C}^{w}\right)$.

We now review the construction of algebraic $K$-theory in preparation for constructing the trace map. Recall Waldhausen's $S_{\bullet}$ construction produces a simplicial Waldhausen category from a Waldhausen category. In the case of a DG-Waldhausen category $\mathcal{C}$, the $S_{\bullet}$ construction produces a simplicial DG-Waldhausen category. Let $\operatorname{Ar}[n]$ denote the category with objects $(i, j)$ for $0 \leq i \leq j \leq n$ and a unique map $(i, j) \rightarrow\left(i^{\prime}, j^{\prime}\right)$ for $i \leq i^{\prime}$ and $j \leq j^{\prime} . S_{n} \mathcal{C}$ is defined to be the full subcategory of the category of functors $A: \operatorname{Ar}[n] \rightarrow \mathcal{C}$ such that 
- $A_{i, i}=0$ for all $i$,

- the map $A_{i, j} \rightarrow A_{i, k}$ is a cofibration (degreewise-split monomorphism) for all $i \leq j \leq k$, and

- the diagram

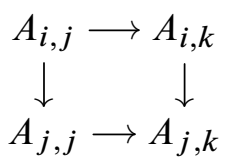

is a pushout square for all $i \leq j \leq k$,

where we write $A_{i, j}$ for $A(i, j)$. The last two conditions can be simplified to the hypothesis that each map $A_{0, j} \rightarrow A_{0, j+1}$ is a cofibration and the induced maps $A_{0, j} / A_{0, i} \rightarrow A_{i, j}$ are isomorphisms. This becomes a DG-Waldhausen category by taking the abelian category to be the category of functors $\operatorname{Ar}[n] \rightarrow A b_{\mathcal{C}}$ and defining a map $A \rightarrow B$ to be a weak equivalence when each $A_{i, j} \rightarrow B_{i, j}$ is a weak equivalence in $\mathcal{C}$. Note that $A \rightarrow B$ is a degreewise-split monomorphism when each $A_{i, j} \rightarrow B_{i, j}$ and each induced map $A_{i, k} \cup_{A_{i, j}} B_{i, j} \rightarrow B_{i, k}$ is a degreewise-split monomorphism. An ordered map $\{1, \ldots, m\} \rightarrow\{1, \ldots, n\}$ induces a functor $\operatorname{Ar}[m] \rightarrow \operatorname{Ar}[n]$ and hence a DG-exact functor $S_{n} \mathcal{C} \rightarrow S_{m} \mathcal{C}$, making $S_{\bullet} \mathcal{C}$ a simplicial DG-Waldhausen category. Because each $S_{p} \mathcal{C}$ is itself a DG-Waldhausen category, the $S_{\bullet}$ construction can be iterated to form multisimplicial DG-Waldhausen categories.

For any DG-Waldhausen category $\mathcal{D}$, let $w_{q} \mathcal{D}$ denote the DG-category whose objects consist of a sequence of $q$ composable weak equivalences in $\mathcal{D}$ (with $w_{0} \mathcal{D}=\mathcal{D}$ ). Using this construction and iterating the $S_{\bullet}$ construction, we obtain multisimplicial DG-categories $w_{\bullet} S_{\bullet}^{(n)} \mathcal{C}$. The inclusion of $\mathcal{D}$ as $S_{1} \mathcal{D}$ induces an $(n+2)$-simplicial map

$$
\mathrm{ob}\left(w_{\bullet} S_{\bullet}^{(n)} \mathcal{C}\right) \wedge S_{\bullet}^{1} \longrightarrow \mathrm{ob}\left(w_{\bullet} S_{\bullet}^{(n+1)} \mathcal{C}\right),
$$

where $S_{\bullet}^{1}$ denotes the standard simplicial model of the circle (with one nondegenerate vertex and one nondegenerate 1-simplex). These structure maps together with the natural $\Sigma_{n}$ action on the categories $w_{q} S_{p}^{(n)} \mathcal{C}$ give the collection of simplicial sets

$$
\left\{\operatorname{diag} \operatorname{ob}\left(w_{\bullet} S_{\bullet}^{(n)} \mathcal{C}\right) \mid n \geq 0\right\}
$$

the structure of a symmetric spectrum. Waldhausen showed that the adjoint attaching maps

$$
\left|\operatorname{diag} \mathrm{ob}\left(w_{\bullet} S_{\bullet}^{(n)} \mathcal{C}\right)\right| \longrightarrow \Omega\left|\operatorname{diag} \mathrm{ob}\left(w_{\bullet} S_{\bullet}^{(n+1)} \mathcal{C}\right)\right|
$$

are weak equivalences for $n>0$; ie, the geometric realization is a positive fibrant symmetric spectrum of topological spaces. 
Definition 10.2 (Waldhausen) $K \mathcal{C}$ is the symmetric spectrum

$$
K \mathcal{C}(n)=\operatorname{diag} \operatorname{ob}\left(w_{\bullet} S_{\bullet}^{(n)} \mathcal{C}\right) .
$$

To mix the $S_{\bullet}$ construction with the cyclic bar construction, we use the more convenient DG-categories $\bar{w}_{q} \mathcal{D}$ in place of the DG-categories $w_{q} \mathcal{D}$. For a DG-Waldhausen category $\mathcal{D}$, let $\bar{w}_{q} \mathcal{D}$ be the DG-category that is the full subcategory of $w_{q} \mathcal{D}$ consisting of those objects where each weak equivalence in the sequence is also a cofibration (degreewise-split monomorphism). The advantage of $\bar{w}_{q} \mathcal{D}$ over $w_{q} \mathcal{D}$ is that the limit defining its mapping complexes is a homotopy limit. Waldhausen also used this construction; the following is a special case of [46, Lemma 1.6.3].

Proposition 10.3 For a $D G-$ Waldhausen category $\mathcal{D}$, the inclusion of $\operatorname{ob}(\bar{w} \bullet \mathcal{D})$ in $\mathrm{ob}\left(w_{\bullet} \mathcal{D}\right)$ is a weak equivalence.

For the construction of the cyclotomic trace, we use the associated spectral category functor of Definition 2.9 to lift the multisimplicial DG-categories $\bar{w}_{\bullet} S_{\bullet}^{(n)} \mathcal{C}$ to multisimplicial spectral categories, which by abuse, we denote with the same notation. For any space $X$, the spaces

$$
\left|T H H\left(\bar{w}_{\bullet} S_{\bullet}^{(n)} \mathcal{C}\right)(X)\right|
$$

then fit together into a symmetric spectrum (indexed on $n$ ) of topological spaces. For each orthogonal $S^{1}$-representation $V$ (q.v., Notation 4.1) let $W T H H(\mathcal{C})(V)$ be the symmetric spectrum defined by

$$
W T H H(\mathcal{C})(V)(n)=\left|\operatorname{THH}\left(\bar{w}_{\bullet} S_{\bullet}^{(n)} \mathcal{C}\right)\left(S^{V}\right)\right| .
$$

As we let $V$ and $n$ vary, $W T H H(C)$ has the structure of a symmetric spectrum in the category of cyclotomic spectra. Let $Q$ be a $\Omega$-spectrum replacement functor in the category of cyclotomic spectra that is enriched in based spaces as an endofunctor (topologizing the mapping spaces in the category of cyclotomic spectra as subspaces of the mapping spaces in the category of orthogonal $S^{1}$-spectra). Then applying $Q$ objectwise in symmetric spectra to the cyclotomic spectra in $W T H H(\mathcal{C})$, we obtain a weakly equivalent symmetric spectrum of cyclotomic spectra where each constituent orthogonal $S^{1}$-spectrum is an $\Omega$-spectrum. Let $W T(\mathcal{C})=Q W T H H(\mathcal{C})$.

The spectrum $W T(\mathcal{C}) \simeq W T H H(\mathcal{C})$ lies between $K \mathcal{C}$ and $C T H H\left(C / \mathcal{C}^{w}\right)$ in the stable category. Write $\bar{K} \mathcal{C}$ for the symmetric spectrum of topological spaces $\bar{K} \mathcal{C}(n)=$ $\left|\mathrm{ob} \bar{w}_{\bullet} S_{\bullet}^{(n)} \mathcal{C}\right|$. We then get a symmetric spectrum of (nonequivariant) orthogonal spectra $F_{0}^{\mathcal{I} S} \bar{K} \mathcal{C}(n)$ representing the same object in the stable category using the free 
functor from spaces to orthogonal spectra. The inclusion of objects (via the identity) induces a map of symmetric spectra of orthogonal spectra

$$
K \mathcal{C} \simeq F_{0}^{\mathcal{I} \mathscr{K}} \bar{K} \mathcal{C} \longrightarrow W H H(\mathcal{C}) \longrightarrow W T(\mathcal{C}),
$$

natural in DG-exact functors. Likewise, using the free functor from spaces to symmetric spectra, we obtain a map of symmetric spectra of orthogonal spectra

$$
T H H(\mathcal{C}) \simeq F_{0}^{\Sigma \varphi} T H H(\mathcal{C}) \longrightarrow W T H H(\mathcal{C}) \longrightarrow W T(\mathcal{C})
$$

natural in DG-exact functors, induced by the identification of $w_{0} S_{\bullet}^{(0)} \mathcal{C}$ as $\mathcal{C}$. Finally, writing $C T\left(\mathcal{C} / \mathcal{C}^{w}\right)$ for the cofiber

$$
C T\left(\mathcal{C} / \mathcal{C}^{w}\right)=C\left(T\left(\mathcal{C}^{w}\right) \rightarrow T(\mathcal{C})\right)
$$

(where, as in Definition 3.8, $T(\mathcal{C})=Q T H H(\mathcal{C})$ ), we obtain the comparison map

$$
C T H H\left(\mathcal{C} / \mathcal{C}^{w}\right) \simeq F_{0}^{\Sigma \varphi} C T\left(\mathcal{C} / \mathcal{C}^{w}\right) \longrightarrow W T(\mathcal{C})
$$

as follows: The functor $\mathcal{C}^{w} \rightarrow \bar{w}_{1} \mathcal{C}$ that sends a $w \mathcal{C}$-acyclic object $a$ to the weak equivalence $0 \rightarrow a$ induces a map from the cone on $T\left(\mathcal{C}^{w}\right)$ to $\left|T\left(w_{\bullet}(\mathcal{C})\right)\right|$ that restricts on the face $T\left(\mathcal{C}^{w}\right)$ to the inclusion of $T\left(\mathcal{C}^{w}\right)$ in $T(\mathcal{C})=T\left(\bar{w}_{0} \mathcal{C}\right)$. This then extends to the map from the cofiber $C T\left(\mathcal{C} / \mathcal{C}^{w}\right)$ above. Similar observations construct the symmetric spectrum of orthogonal spectra

$$
W T C(\mathcal{C})=\left|T C\left(w_{\bullet} S_{\bullet}^{(-)} \mathcal{C}\right)\right|
$$

and maps

$$
K \mathcal{C} \simeq F_{0}^{\mathcal{I} \varphi} \bar{K} \mathcal{C} \longrightarrow W T C(\mathcal{C}) \longleftarrow F_{0}^{\Sigma \varphi} C T C\left(\mathcal{C} / \mathcal{C}^{w}\right) \simeq C T C\left(\mathcal{C} / \mathcal{C}^{w}\right)
$$

The following is the main theorem of this section and is proved below.

Theorem 10.4 For a $D G-$ Waldhausen category $\mathcal{C}$, the maps

$$
F_{0}^{\Sigma \mathscr{S}} \mathrm{CTHH}\left(\mathcal{C} / \mathcal{C}^{w}\right) \longrightarrow W T H H(\mathcal{C}) \quad \text { and } \quad F_{0}^{\Sigma \varphi} C T C\left(\mathcal{C} / \mathcal{C}^{w}\right) \longrightarrow W T C(\mathcal{C})
$$

are level equivalences of symmetric spectra of orthogonal spectra.

We can now define the trace. 
Definition 10.5 The cyclotomic trace maps from $K$-theory to $T C$ and from $K$-theory to $T H H$ are the zigzags

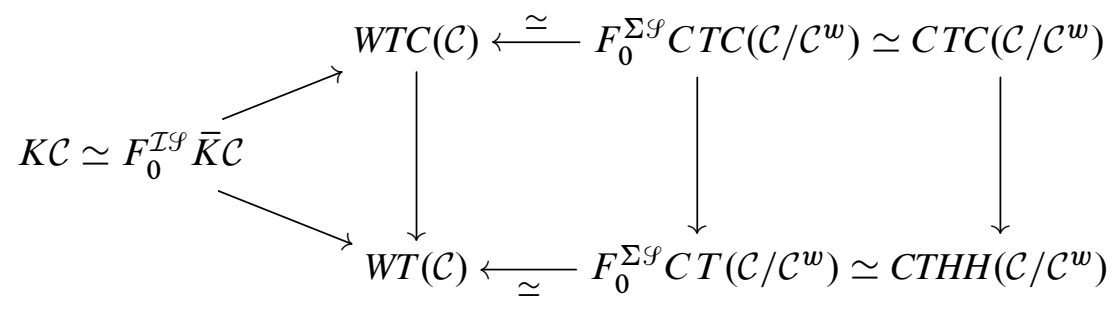

Every map in the diagram is natural in DG-exact functors.

When we restrict to appropriate categories of schemes or pairs of schemes as in [43, Section 6], we can factor the trace above through nonconnective $K$-theory. Essentially, we take $\bar{K}$ and $W T$ as our model functors to spectra (which here would be the pointset category of symmetric spectra of orthogonal spectra) applied to the appropriate DG-Waldhausen category model for perfect complexes (as in [43, Section 3]), depending on the kind of naturality required for the maps of schemes. For any of these models, we get natural pairings

$$
\bar{K}(X \text { on }(X-U)) \wedge K_{f}\left(\mathbb{Z}\left[t, t^{-1}\right]\right) \longrightarrow \bar{K}\left(X\left[t, t^{-1}\right] \text { on }\left(X\left[t, t^{-1}\right]-U\left[t, t^{-1}\right]\right)\right),
$$

$W T H H(X$ on $(X-U)) \wedge K_{f}\left(\mathbb{Z}\left[t, t^{-1}\right]\right)$

$$
\longrightarrow W T H H\left(X\left[t, t^{-1}\right] \text { on }\left(X\left[t, t^{-1}\right]-U\left[t, t^{-1}\right]\right)\right),
$$

where $K_{f}\left(\mathbb{Z}\left[t, t^{-1}\right]\right)$ denotes the Waldhausen $K$-theory symmetric ring spectrum of the exact category with objects the canonical free modules

$$
0, \quad \mathbb{Z}\left[t, t^{-1}\right], \quad\left(\mathbb{Z}\left[t, t^{-1}\right]\right)^{2}, \quad\left(\mathbb{Z}\left[t, t^{-1}\right]\right)^{3}, \ldots .
$$

The arguments presented in Section 9 extend to this context to construct the nonconnective cyclotomic trace.

The remainder of the section proves Theorem 10.4. A version of the Additivity Theorem, as always, provides the key lemma. Given DG-Waldhausen categories $\mathcal{A}, \mathcal{B}, \mathcal{C}$ and DG-exact functors $\phi: \mathcal{A} \rightarrow \mathcal{B}, \psi: \mathcal{C} \rightarrow \mathcal{B}$, let $\mathcal{E}(\mathcal{A}, \mathcal{B}, \mathcal{C})$ be the DG-Waldhausen category where an object consists of

(i) a tuple $(a, b, c)$ of objects $a \in \mathcal{A}, b \in \mathcal{B}$, and $c \in \mathcal{C}$, and

(ii) a degreewise-split short exact sequence in $\mathcal{B}$,

$$
0 \longrightarrow \phi a \longrightarrow b \longrightarrow \psi c \longrightarrow 0 .
$$


The mapping complex in $\mathcal{E}(\mathcal{A}, \mathcal{B}, \mathcal{C})$ from $(a, b, c)$ to $\left(a^{\prime}, b^{\prime}, c^{\prime}\right)$ is

$$
\left(\mathcal{A}\left(a, a^{\prime}\right) \times_{\mathcal{B}\left(\phi a, b^{\prime}\right)} \mathcal{B}\left(b, b^{\prime}\right)\right) \times_{\mathcal{B}\left(\psi c, \psi c^{\prime}\right)} \mathcal{C}\left(c, c^{\prime}\right),
$$

which is isomorphic to

$$
\mathcal{A}\left(a, a^{\prime}\right) \times_{\mathcal{B}\left(\phi a, \phi a^{\prime}\right)}\left(\mathcal{B}\left(b, b^{\prime}\right) \times_{\mathcal{B}\left(b, \psi c^{\prime}\right)} \mathcal{C}\left(c, c^{\prime}\right)\right) .
$$

Note that each of the maps

$$
\begin{gathered}
\mathcal{B}\left(b, b^{\prime}\right) \longrightarrow \mathcal{B}\left(\phi a, b^{\prime}\right), \\
\mathcal{B}\left(b, b^{\prime}\right) \longrightarrow \mathcal{B}\left(b, \psi c^{\prime}\right), \\
\mathcal{A}\left(a, a^{\prime}\right) \times_{\mathcal{B}\left(\phi a, b^{\prime}\right)} \mathcal{B}\left(b, b^{\prime}\right) \longrightarrow \mathcal{B}\left(\psi c, \psi c^{\prime}\right), \\
\mathcal{B}\left(b, b^{\prime}\right) \times_{\mathcal{B}\left(b, \psi c^{\prime}\right)} \mathcal{C}\left(c, c^{\prime}\right) \longrightarrow \mathcal{B}\left(\phi a, \phi a^{\prime}\right)
\end{gathered}
$$

is a degreewise-split epimorphism, and so the limits in (10.6) and (10.7) are homotopy limits.

We have DG-exact functors

$$
\begin{aligned}
& \alpha: \mathcal{E}(\mathcal{A}, \mathcal{B}, \mathcal{C}) \longrightarrow \mathcal{A}, \\
& \beta: \mathcal{E}(\mathcal{A}, \mathcal{B}, \mathcal{C}) \longrightarrow \mathcal{B}, \\
& \gamma: \mathcal{E}(\mathcal{A}, \mathcal{B}, \mathcal{C}) \longrightarrow \mathcal{C},
\end{aligned}
$$

induced by the forgetful functor and a DG-exact functor

$$
\sigma: \mathcal{A} \times \mathcal{C} \longrightarrow \mathcal{E}(\mathcal{A}, \mathcal{B}, \mathcal{C})
$$

induced by $\sigma(a, c)=(a, \phi a \oplus \psi c, c)$ (and the split short exact sequence). The version of the additivity theorem we prove compares the maps induced on $T H H$ by $\sigma$ and $\alpha \vee \gamma$.

Theorem 10.8 (Additivity Theorem) The functors

$$
\operatorname{THH}(\mathcal{A}) \vee \operatorname{THH}(\mathcal{C}) \longrightarrow \operatorname{THH}(\mathcal{E}(\mathcal{A}, \mathcal{B}, \mathcal{C})) \longrightarrow T H H(\mathcal{A}) \times T H H(\mathcal{C})
$$

induced by $\sigma$ and $\alpha \times \gamma$ are inverse weak equivalences.

Proof Consider the DG-exact functor $\phi^{\prime}: \mathcal{A} \rightarrow \mathcal{E}(\mathcal{A}, \mathcal{B}, \mathcal{C})$ that takes $a$ in $\mathcal{A}$ to $(a, \phi a, 0)$. By (10.6), we see that this is a DK-embedding. Now by Theorem 7.1, it suffices to show that the functor $\psi^{\prime}: \mathcal{B} \rightarrow \mathcal{E}(\mathcal{A}, \mathcal{B}, \mathcal{C})$ (sending $c$ to $(0, \psi c, c)$ ) induces an equivalence from homotopy category $\pi_{0} \mathcal{B}$ to the triangulated quotient $\pi_{0} \mathcal{E}(\mathcal{A}, \mathcal{B}, \mathcal{C}) / \pi_{0} \mathcal{A}$. This is a straightforward calculation from (10.7). 
We can apply this to understand the effect both of $\bar{w}_{q}$ and $S_{p}$ on $T H H$. An element of $\bar{w}_{q}$ of $\mathcal{C}$ is a sequence of degreewise-split maps

$$
c_{0} \longrightarrow \cdots \longrightarrow c_{q}
$$

such that each quotient $c_{i} / c_{i-1}$ is in $\mathcal{C}^{w}$. Choosing quotients, we get a DK-equivalent DG-category $\bar{W}_{q}$ that is a DG-Waldhausen category. Furthermore, we can identify $\bar{W}_{q+1} \mathcal{C}$ as $\mathcal{E}\left(\bar{W}_{q} \mathcal{C}, \mathcal{C}, \mathcal{C}^{w}\right)$, for the functor $\phi: \bar{W}_{q} \mathcal{C} \rightarrow \mathcal{C}$ that sends the sequence pictured above to $c_{q}$. As a consequence we get the following corollary.

Corollary 10.9 For all $q$, the map

$$
\underbrace{\operatorname{THH}\left(\mathcal{C}^{w}\right) \vee \cdots \vee T H H\left(\mathcal{C}^{w}\right)}_{q \text { factors }} \vee \operatorname{THH}(\mathcal{C}) \longrightarrow T H H\left(\bar{w}_{q} \mathcal{C}\right)
$$

induced by the map that sends $\left(a_{1}, \ldots, a_{q}, c\right)$ to

$$
c \longrightarrow c \oplus a_{1} \longrightarrow \cdots \longrightarrow c \oplus a_{1} \oplus \cdots \oplus a_{q}
$$

is a weak equivalence.

Similarly, for any DG-Waldhausen category $\mathcal{D}$, the DG-Waldhausen category $S_{r} \mathcal{D}$ is DK-equivalent (via a DG-exact functor) to the DG-category $\mathcal{E}\left(S_{r-1} \mathcal{D}, \mathcal{D}, \mathcal{D}\right)$ for the functor $\phi: S_{r} \mathcal{D} \rightarrow \mathcal{D}$ that takes $\left\{A_{i, j}\right\}$ to $A_{0, r-1}$. We use this observation to prove the following corollary.

Corollary 10.10 For each $n$ and $q$, the map

$$
\Sigma\left|T H H\left(\bar{w}_{q} S_{\bullet}^{(n)} \mathcal{C}\right)\right| \longrightarrow\left|T H H\left(\bar{w}_{q} S_{\bullet}^{(n+1)} \mathcal{C}\right)\right|
$$

is a weak equivalence.

Proof We can write $\Sigma\left|T H H\left(\bar{w}_{q} S_{\bullet}^{(n)} \mathcal{C}\right)\right|$ as the geometric realization of a multisimplicial object with one more simplicial direction, $\operatorname{THH}\left(\bar{w}_{q} S_{\bullet}^{(n)} \mathcal{C}\right) \wedge S_{\bullet}^{1}$, where $S_{\bullet}^{1}$ denotes the standard simplicial model of the circle. The map in the statement is induced by the map on geometric realizations of the map of multisimplical objects

$$
\operatorname{THH}\left(\bar{w}_{q} S_{\bullet}^{(n)} \mathcal{C}\right) \wedge S_{\bullet}^{1} \longrightarrow \operatorname{THH}\left(\bar{w}_{q} S_{\bullet}^{(n+1)} \mathcal{C}\right)
$$

Using the standard isomorphisms

$$
S_{r} \bar{w}_{q} \cong \bar{w}_{q} S_{r}, \quad S_{r} S_{p} \cong S_{p} S_{r}
$$


and writing $\mathcal{D}=\bar{w}_{q} S_{p_{1}} \cdots S_{p_{n}} \mathcal{C}$, we are looking at maps of the form

$$
\bigvee_{r} \operatorname{THH}(\mathcal{D}) \longrightarrow \operatorname{THH}\left(S_{r} \mathcal{D}\right) \text {. }
$$

Using the relationship of $S_{r} \mathcal{D}$ and $\mathcal{E}\left(S_{r-1} \mathcal{D}, \mathcal{D}, \mathcal{D}\right)$ as above, we see by induction that this map is a weak equivalence.

Combining these two corollaries, we prove Theorem 10.4.

Proof of Theorem 10.4 We can identify the map $\operatorname{CTHH}\left(\mathcal{C} / \mathcal{C}^{w}\right) \rightarrow\left|T H H\left(\bar{w}_{\bullet} \mathcal{C}^{w}\right)\right|$ above as the induced map on geometric realization of the map of simplicial objects

$$
\underbrace{\operatorname{THH}\left(\mathcal{C}^{w}\right) \vee \cdots \vee \operatorname{THH}\left(\mathcal{C}^{w}\right)}_{\bullet \text { factors }} \vee \operatorname{THH}(\mathcal{C}) \longrightarrow \operatorname{THH}\left(\bar{w}_{\bullet} \mathcal{C}\right),
$$

and is a weak equivalence by Corollary 10.9. For $n>0$, the $n$-th level of the symmetric spectrum of orthogonal spectra $F_{0}^{\Sigma \mathscr{S}} \mathrm{CTHH}\left(\mathcal{C} / \mathcal{C}^{w}\right)$ is $\Sigma^{n} \mathrm{CTHH}\left(\mathcal{C} / \mathcal{C}^{w}\right)$. It now follows from Corollary 10.10, that the map

$$
F_{0}^{\Sigma \varphi} C T H H\left(C / \mathcal{C}^{w}\right) \longrightarrow W T H H(C)
$$

is a level equivalence of symmetric spectra of orthogonal spectra and Theorem 10.4 follows.

\section{$11 T H H$ and $T C$ of small spectral model categories}

Our treatment of $T H H$ and $T C$ of spectral categories in the rest of the paper took the perspective that all the homotopy information is encoded in the mapping spectra. In the context of closed model categories enriched over symmetric spectra, the weak equivalences encode an additional localization. We can extract a spectral category satisfying the hypotheses of the main discussion of the paper from such a model category by restricting to the full spectral subcategory of cofibrant-fibrant objects. However, this subcategory is not usually preserved by naturally occurring functors between model categories, which tend to preserve only cofibrant or only fibrant objects.

In this section, we present a construction of $T H H$ of a small spectral model category in terms of either the full subcategory of cofibrant or fibrant objects. (Here we must use the original convention of Quillen that closed model categories are closed under finite limits and colimits rather than the modern convention that they are closed under all small limits and colimits.) The construction is in terms of a "cofiber $T H H$ " description, exactly as in the $T H H$ of localization pairs constructed in Section 7. Since the quotient 
of the subcategory of cofibrants by the acyclic cofibrants is the homotopy category of the model category, we can regard this pair as analogous to a localization pair, although it may not satisfy the hypotheses of the definition. Nevertheless, a similar (but easier) proof applies to compare the $T H H$ of this pair to the $T H H$ of the cofibrant-fibrants. The main theorem of this section is the following.

Theorem 11.1 Let $\mathcal{M}$ be a small closed model category enriched over symmetric spectra, satisfying the symmetric spectrum version of SM7. Write $\mathcal{A}$ for the subcategory of acyclic objects (objects weakly equivalent to the zero object), and subscripts $c$ and $f$ for the subcategories of cofibrant and fibrant objects, respectively, of $\mathcal{M}$ and likewise of $\mathcal{A}$. In the following diagram, the vertical map is always a weak equivalence, the left-hand map is a weak equivalence if $\mathcal{M}$ is left proper, and the right-hand map is a weak equivalence if $\mathcal{M}$ is right proper.

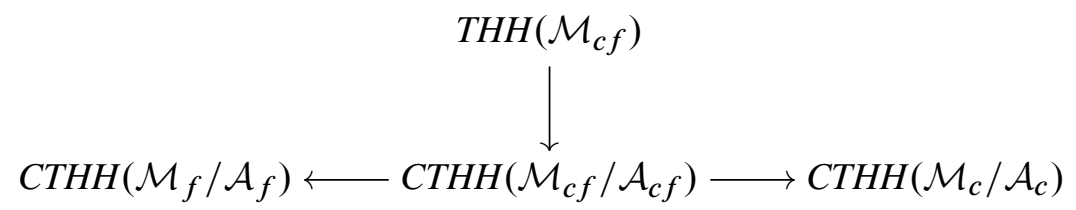

Since for any pair of objects in $\mathcal{A}_{c f}$, the symmetric spectrum of maps is homotopically trivial, $T H H\left(\mathcal{A}_{c f}\right)$ is homotopically trivial, and it then follows that the vertical map is a weak equivalence.

Of the remaining statements in the theorem, we treat the case of the right horizontal map in detail; the case of the left horizontal map is similar (and in fact follows by considering the opposite category). Let $\mathcal{M}^{\prime}=\mathcal{M}^{\text {Cell }}$ be the pointwise cofibrant spectral category weakly equivalent to $\mathcal{M}$ from Proposition 2.7 , and let $\mathcal{M}_{c}^{\prime}, \mathcal{A}^{\prime}$, and $\mathcal{A}_{c}^{\prime}$ be the appropriate subcategories. As in Section 7 , we define the $\left(\mathcal{M}_{c}^{\prime}, \mathcal{M}_{c}^{\prime}\right)-$ bimodule $\mathcal{L}_{\mathcal{A}_{c}}^{\mathcal{M}_{c}}$ by

$$
\mathcal{L}_{\mathcal{A}_{c}}^{\mathcal{M}_{c}}(x, y)=B\left(\mathcal{M}_{c}^{\prime}(-, y) ; \mathcal{A}_{c}^{\prime} ; \mathcal{M}_{c}^{\prime}(x,-)\right)
$$

and $\mathcal{Q}_{\mathcal{A}_{c}}^{\mathcal{M}_{c}}$ as the cofiber of the composition map $\mathcal{L}_{\mathcal{A}_{c}}^{\mathcal{M}_{c}} \rightarrow \mathcal{M}_{c}^{\prime}$. The following lemma lists the properties of $\mathcal{Q}_{\mathcal{A}_{c}}^{\mathcal{M}_{c}}$ we need in the proof of the theorem.

Lemma 11.2 Let $x$ be an object of $\mathcal{M}_{c}$.

(i) For $y$ in $\mathcal{M}_{c f}$, the map $\mathcal{M}_{c}^{\prime}(x, y) \rightarrow \mathcal{Q}_{\mathcal{A}_{c}}^{\mathcal{M}_{c}}(x, y)$ is a weak equivalence.

(ii) If $\mathcal{M}$ is right proper, then $\mathcal{Q}_{\mathcal{A}_{c}}^{\mathcal{M}_{c}}(x,-)$ preserves weak equivalences. 
Proof Since the mapping spectrum from a cofibrant acyclic object to a fibrant object is homotopically trivial, for any object $y$ in $\mathcal{M}_{c f}, \mathcal{L}_{\mathcal{A}_{c}}^{\mathcal{M}_{c}}(x, y)$ is homotopically trivial, and the map $\mathcal{M}_{c}^{\prime}(x, y) \rightarrow \mathcal{Q}_{\mathcal{A}_{c}}^{\mathcal{M}_{c}}(x, y)$ is a weak equivalence. This proves (i). To prove (ii), it suffices to show that for any object $y$ and any fibrant replacement $y \rightarrow y^{\prime}$, the map $\mathcal{Q}_{\mathcal{A}_{c}}^{\mathcal{M}_{c}}(x, y) \rightarrow \mathcal{Q}_{\mathcal{A}_{c}}^{\mathcal{M}_{c}}\left(x, y^{\prime}\right)$ is a weak equivalence. Factor the initial map $* \rightarrow y^{\prime}$ as an acyclic cofibration followed by a fibration $a^{\prime} \rightarrow y^{\prime}$, and let $a$ be a cofibrant replacement of the pullback $y \times y^{\prime} a^{\prime}$.

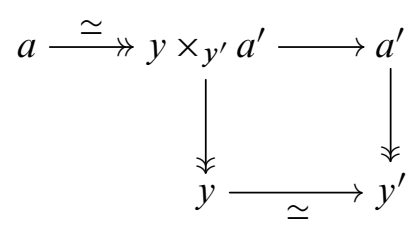

We obtain from this fibration pullback square (and the symmetric spectrum version of SM7) the homotopy (co)cartesian square of $\mathcal{M}_{c}$-modules on the left below, and from this, the homotopy cocartesian square of $\mathcal{M}_{c}^{\prime}$-modules on the right below.
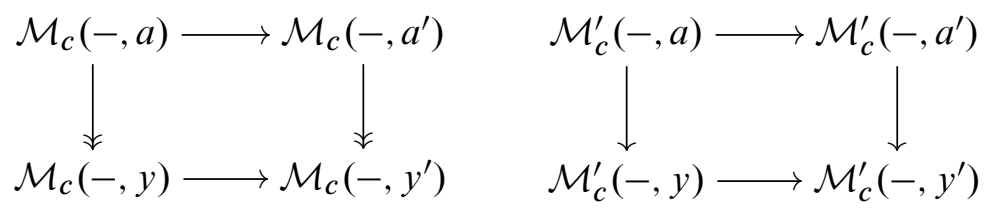

Looking at the construction of $\mathcal{L}_{\mathcal{A}_{c}}^{\mathcal{M}_{c}}$, the bar construction preserves homotopy cocartesian squares in either variable, and so we see that the square on the left below is homotopy cocartesian; it follows that the square on the right below is homotopy cocartesian.
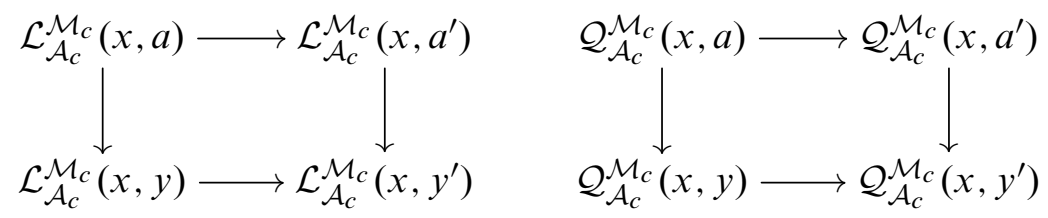

The hypothesis that $\mathcal{M}$ is right proper implies that the map $a \rightarrow a^{\prime}$ is a weak equivalence and therefore that $a$ is in $\mathcal{A}_{c}$. It follows that $\mathcal{Q}_{\mathcal{A}_{c}}^{\mathcal{M}_{c}}(x, a)$ and $\mathcal{Q}_{\mathcal{A}_{c}}^{\mathcal{M}_{c}}\left(x, a^{\prime}\right)$ are homotopically trivial, and that $\mathcal{Q}_{\mathcal{A}_{c}}^{\mathcal{M}_{c}}(x, y) \rightarrow \mathcal{Q}_{\mathcal{A}_{c}}^{\mathcal{M}_{c}}\left(x, y^{\prime}\right)$ is a weak equivalence.

As in Section 7, we have a natural weak equivalence relating

$$
\operatorname{CTHH}\left(\mathcal{M}_{c} / \mathcal{A}_{c}\right) \simeq \operatorname{CTHH}\left(\mathcal{M}_{c}^{\prime} / \mathcal{A}_{c}^{\prime}\right)
$$

with $\operatorname{THH}\left(\mathcal{M}_{c} ; \mathcal{Q}_{\mathcal{A}_{c}}^{\mathcal{M}_{c}}\right)$, compatible with the map from $T H H\left(\mathcal{M}_{c}^{\prime}\right) \simeq T H H\left(\mathcal{M}_{c}\right)$. Applying part (i) of the lemma, to complete the proof of the theorem, it suffices to show 
that the map

$$
\operatorname{THH}\left(\mathcal{M}_{c f}^{\prime} ; \mathcal{Q}_{\mathcal{A}_{c}}^{\mathcal{M}_{c}}\right) \longrightarrow \operatorname{THH}\left(\mathcal{M}_{c}^{\prime} ; \mathcal{Q}_{\mathcal{A}_{c}}^{\mathcal{M}_{c}}\right)
$$

is a weak equivalence.

Our strategy as in Section 7 is to apply the Dennis-Waldhausen Morita argument (Proposition 6.2) to reduce to proving an objectwise statement. For this, we use the weak equivalence of $\left(\mathcal{M}_{c}^{\prime}, \mathcal{M}_{c}^{\prime}\right)$-bimodules $B\left(\mathcal{M}_{c}^{\prime} ; \mathcal{M}_{c}^{\prime} ; \mathcal{Q}_{\mathcal{A}_{c}}^{\mathcal{M}_{c}}\right) \rightarrow \mathcal{Q}_{\mathcal{A}_{c}}^{\mathcal{M}_{c}}$. Then the Dennis-Waldhausen Morita argument gives us a weak equivalence

$$
\operatorname{THH}\left(\mathcal{M}_{c f}^{\prime} ; B\left(\mathcal{M}_{c}^{\prime} ; \mathcal{M}_{c}^{\prime} ; \mathcal{Q}_{\mathcal{A}_{c}}^{\mathcal{M}_{c}}\right)\right) \simeq \operatorname{THH}\left(\mathcal{M}_{c}^{\prime} ; B\left(\mathcal{Q}_{\mathcal{A}_{c}}^{\mathcal{M}_{c}} ; \mathcal{M}_{c f}^{\prime} ; \mathcal{M}_{c}^{\prime}\right)\right)
$$

and likewise the analogous weak equivalence with $\mathcal{M}_{c f}^{\prime}$ replaced by $\mathcal{M}_{c}^{\prime}$. Now it suffices to show that the map

$$
\operatorname{THH}\left(\mathcal{M}_{c}^{\prime} ; B\left(\mathcal{Q}_{\mathcal{A}_{c}}^{\mathcal{M}_{c}} ; \mathcal{M}_{c f}^{\prime} ; \mathcal{M}_{c}^{\prime}\right)\right) \longrightarrow \operatorname{THH}\left(\mathcal{M}_{c}^{\prime} ; B\left(\mathcal{Q}_{\mathcal{A}_{c}}^{\mathcal{M}_{c}} ; \mathcal{M}_{c}^{\prime} ; \mathcal{M}_{c}^{\prime}\right)\right)
$$

is a weak equivalence. This map is induced by the map of $\left(\mathcal{M}_{c}^{\prime}, \mathcal{M}_{c}^{\prime}\right)$-bimodules

$$
B\left(\mathcal{Q}_{\mathcal{A}_{c}}^{\mathcal{M}_{c}} ; \mathcal{M}_{c f}^{\prime} ; \mathcal{M}_{c}^{\prime}\right) \longrightarrow B\left(\mathcal{Q}_{\mathcal{A}_{c}}^{\mathcal{M}_{c}} ; \mathcal{M}_{c}^{\prime} ; \mathcal{M}_{c}^{\prime}\right)
$$

and so it suffices to show that the map

$$
B\left(\mathcal{Q}_{\mathcal{A}_{c}}^{\mathcal{M}_{c}}(-, y) ; \mathcal{M}_{c f}^{\prime} ; \mathcal{M}_{c}^{\prime}(x,-)\right) \longrightarrow \mathcal{Q}_{\mathcal{A}_{c}}^{\mathcal{M}_{c}}(x, y)
$$

is a weak equivalence for each pair of objects $x, y$ in $\mathcal{M}_{c}$. This is clear from the Two-Sided Bar Construction Lemma and part (i) of the lemma when $y$ is in $\mathcal{M}_{c f}$. It then follows for arbitrary $y$ by part (ii) of the lemma.

\section{References}

[1] S Bloch, S Lichtenbaum, A spectral sequence for motivic cohomology, preprint (1995) Available at http://www.math.uiuc.edu/K-theory/0062/

[2] A J Blumberg, MA Mandell, Algebraic K-theory and abstract homotopy theory, Adv. Math. 226 (2011) 3760-3812 MR2764905

[3] M Bökstedt, Topological Hochschild homology, Bielefeld preprint (1988)

[4] M Bökstedt, W C Hsiang, I Madsen, The cyclotomic trace and algebraic K-theory of spaces, Invent. Math. 111 (1993) 465-539 MR1202133

[5] A I Bondal, M Larsen, V A Lunts, Grothendieck ring of pretriangulated categories, Int. Math. Res. Not. 2004 (2004) 1461-1495 MR2051435

[6] A K Bousfield, The localization of spaces with respect to homology, Topology 14 (1975) 133-150 MR0380779 
[7] G Cortiñas, Infinitesimal K-theory, J. Reine Angew. Math. 503 (1998) 129-160 MR1650347

[8] G Cortiñas, C Haesemeyer, M Schlichting, C Weibel, Cyclic homology, cdhcohomology and negative $K$-theory, Ann. of Math. 167 (2008) 549-573 MR2415380

[9] G Cortiñas, C Haesemeyer, C Weibel, $K$-regularity, cdh-fibrant Hochschild homology, and a conjecture of Vorst, J. Amer. Math. Soc. 21 (2008) 547-561 MR2373359

[10] V Drinfeld, DG quotients of DG categories, J. Algebra 272 (2004) 643-691 MR2028075

[11] D Dugger, Spectral enrichments of model categories, Homology, Homotopy Appl. 8 (2006) 1-30 MR2205213

[12] D Dugger, B Shipley, Enriched model categories and an application to additive endomorphism spectra, Theory Appl. Categ. 18 (2007) 400-439 MR2342167

[13] B I Dundas, R McCarthy, Topological Hochschild homology of ring functors and exact categories, J. Pure Appl. Algebra 109 (1996) 231-294 MR1388700

[14] A D Elmendorf, I Kriz, MA Mandell, J P May, Rings, modules, and algebras in stable homotopy theory, Math.Surveys and Monogr. 47, Amer. Math. Soc. (1997) MR1417719 With an appendix by M Cole

[15] E M Friedlander, A Suslin, The spectral sequence relating algebraic $K$-theory to motivic cohomology, Ann. Sci. École Norm. Sup. 35 (2002) 773-875 MR1949356

[16] T Geisser, L Hesselholt, Topological cyclic homology of schemes, from: "Algebraic $K$-theory (Seattle, WA, 1997)”, (W Raskind, C Weibel, editors), Proc. Sympos. Pure Math. 67, Amer. Math. Soc., Providence, RI (1999) 41-87 MR1743237

[17] T Geisser, L Hesselholt, On the vanishing of negative $K$-groups, Math. Ann. 348 (2010) 707-736 MR2677901

[18] T G Goodwillie, Relative algebraic K-theory and cyclic homology, Ann. of Math. 124 (1986) 347-402 MR855300

[19] L Hesselholt, I Madsen, Cyclic polytopes and the $K$-theory of truncated polynomial algebras, Invent. Math. 130 (1997) 73-97 MR1471886

[20] L Hesselholt, I Madsen, On the K-theory of finite algebras over Witt vectors of perfect fields, Topology 36 (1997) 29-101 MR1410465

[21] L Hesselholt, I Madsen, On the K-theory of local fields, Ann. of Math. 158 (2003) 1-113 MR1998478

[22] PS Hirschhorn, Model categories and their localizations, Math. Surveys and Monogr. 99, Amer. Math. Soc. (2003) MR1944041

[23] M Hovey, J H Palmieri, N P Strickland, Axiomatic stable homotopy theory, 128, no. 610, Amer. Math. Soc. (1997) MR1388895 
[24] M Hovey, B Shipley, J Smith, Symmetric spectra, J. Amer. Math. Soc. 13 (2000) 149-208 MR1695653

[25] B Keller, On the cyclic homology of exact categories, J. Pure Appl. Algebra 136 (1999) 1-56 MR1667558

[26] G M Kelly, Basic concepts of enriched category theory, London Math. Soc. Lecture Note Series 64, Cambridge Univ. Press (1982) MR651714

[27] T A Kro, Model structure on operads in orthogonal spectra, Homology, Homotopy Appl. 9 (2007) 397-412 MR2366955

[28] M Levine, The homotopy coniveau tower, J. Topol. 1 (2008) 217-267 MR2365658

[29] L G Lewis, Jr, Is there a convenient category of spectra?, J. Pure Appl. Algebra 73 (1991) 233-246 MR1124786

[30] L G Lewis, Jr, J P May, M Steinberger, J E McClure, Equivariant stable homotopy theory, Lecture Notes in Math. 1213, Springer, Berlin (1986) MR866482 With contributions by $\mathrm{J}$ E McClure

[31] M A Mandell, J P May, Equivariant orthogonal spectra and $S$-modules, 159, no. 755, Amer. Math. Soc. (2002) MR1922205

[32] M A Mandell, J P May, S Schwede, B Shipley, Model categories of diagram spectra, Proc. London Math. Soc. 82 (2001) 441-512 MR1806878

[33] J P May, The geometry of iterated loop spaces, Lectures Notes in Math. 271, Springer, Berlin (1972) MR0420610

[34] J P May, Equivariant homotopy and cohomology theory, CBMS Regional Conference Series in Math. 91, Conference Board of the Math. Sciences, Washington, DC (1996) MR1413302 With contributions by M Cole, G Comezaña, S Costenoble, A D Elmendorf, J P C Greenlees, L G Lewis, Jr., R J Piacenza, G Triantafillou and S Waner

[35] R McCarthy, Relative algebraic $K$-theory and topological cyclic homology, Acta Math. 179 (1997) 197-222 MR1607555

[36] A Neeman, The connection between the $K$-theory localization theorem of Thomason, Trobaugh and Yao and the smashing subcategories of Bousfield and Ravenel, Ann. Sci. École Norm. Sup. 25 (1992) 547-566 MR1191736

[37] S Schwede, B Shipley, Equivalences of monoidal model categories, Algebr. Geom. Topol. 3 (2003) 287-334 MR1997322

[38] S Schwede, B Shipley, Stable model categories are categories of modules, Topology 42 (2003) 103-153 MR1928647

[39] B Shipley, Symmetric spectra and topological Hochschild homology, K-Theory 19 (2000) 155-183 MR1740756

[40] B Shipley, HZ-algebra spectra are differential graded algebras, Amer. J. Math. 129 (2007) 351-379 MR2306038 
[41] R W Thomason, Algebraic K-theory and étale cohomology, Ann. Sci. École Norm. Sup. 18 (1985) 437-552 MR826102

[42] R W Thomason, Les $K$-groupes d'un schéma éclaté et une formule d'intersection excédentaire, Invent. Math. 112 (1993) 195-215 MR1207482

[43] R W Thomason, T Trobaugh, Higher algebraic $K$-theory of schemes and of derived categories, from: “The Grothendieck Festschrift, Vol. III”, (P Cartier, L Illusie, N M Katz, G Laumon, K A Ribet, editors), Progr. Math. 88, Birkhäuser, Boston, MA (1990) 247-435 MR1106918

[44] B Toën, G Vezzosi, A remark on $K$-theory and $S$-categories, Topology 43 (2004) 765-791 MR2061207

[45] F Waldhausen, Algebraic K-theory of topological spaces. II, from: "Algebraic topology, Aarhus 1978 (Proc. Sympos., Univ. Aarhus, 1978)”, (J L Dupont, I H Madsen, editors), Lecture Notes in Math. 763, Springer, Berlin (1979) 356-394 MR561230

[46] F Waldhausen, Algebraic $K$-theory of spaces, from: "Algebraic and geometric topology (New Brunswick, NJ, 1983)”, (A Ranicki, N Levitt, F Quinn, editors), Lecture Notes in Math. 1126, Springer, Berlin (1985) 318-419 MR802796

Department of Mathematics, University of Texas at Austin

1 University Station C1200, Austin TX 78712, USA

Department of Mathematics, Indiana University

Rawles Hall, 831 E 3rd St, Bloomington IN 47405, USA

blumberg@math.utexas.edu, mmandell@indiana.edu

Proposed: Ralph Cohen

Seconded: Haynes Miller, Jesper Grodal
Received: 18 November 2010 Revised: 7 February 2012 